doi:10.1017/S1474748020000559 C The Author(s), 2021. Published by Cambridge University Press. This is an Open Access article, distributed under the terms of the Creative Commons Attribution licence (http://creativecommons.org/licenses/by/4.0/), which permits unrestricted re-use, distribution, and reproduction in any medium, provided the original work is properly cited.

\title{
SFT COMPUTATIONS AND INTERSECTION THEORY IN HIGHER-DIMENSIONAL CONTACT MANIFOLDS
}

\author{
AGUSTIN MORENO \\ Uppsala Universitet, Uppsala, Sweden \\ (Received 1 March 2019; revised 9 August 2020; accepted 14 August 2020; first \\ published online 25 January 2021)

\begin{abstract}
I construct infinitely many nondiffeomorphic examples of 5-dimensional contact manifolds which are tight, admit no strong fillings and do not have Giroux torsion. I obtain obstruction results for symplectic cobordisms, for which I give a proof not relying on the polyfold abstract perturbation scheme for Symplectic Field Theory (SFT). These results are part of my PhD thesis [23], and are the first applications of higher-dimensional Siefring intersection theory for holomorphic curves and hypersurfaces, as outlined in $[23,24]$, as a prequel to [30].
\end{abstract}

Keywords: Contact Topology, Symplectic Geometry, Holomorphic Curves, Symplectic Field Theory.

2020 Mathematics subject classification: Primary 53D42

Secondary 53D10, 53D35

\section{Contents}

1 Introduction

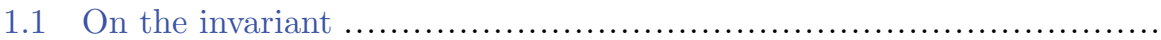

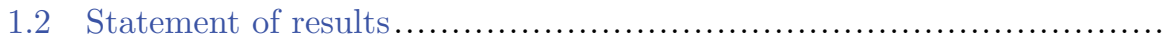

2 Guide to the article

2.1 Basic notions.....................................................

3 Model contact manifolds: A dual point of view $\quad \mathbf{1 2 3 0}$

3.1 Construction of the model contact manifolds ....................... 1230

3.2 Deformation to an SHS along the cylindrical region................... 1236

3.3 Compatible almost complex structure............................. 1237

3.4 Foliation by holomorphic hypersurfaces ............................ 1238

3.5 Index computations ............................................... 1241

3.6 Holomorphic curves lie in hypersurfaces ........................... 1242

3.7 Regularity inside a hypersurface versus regularity in the symplectisation for curves of genus zero ......................................... 1243

3.8 Obstruction bundles ............................................. 1245

4 Nonfillable 5-dimensional model with no Giroux torsion $\quad \mathbf{1 2 5 0}$ 
4.1 Curves on symplectisation of prequantisation spaces: Existence and uniqueness .................................................... 1251

4.2 Some remarks and useful facts .................................... 1252

4.3 Investigating 1 -torsion .......................................... 1253

4.4 The sporadic configuration ........................................ 1259

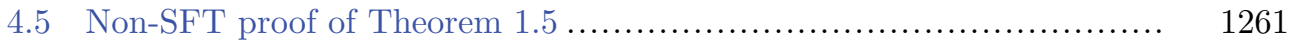

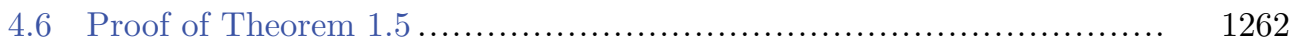

B.1 The twisted contact structures .................................. 1268

\section{Introduction}

This article is a followup to [18], where I address the general problem of constructing 'interesting' families of contact structures in higher dimensions, together with developing general computational techniques for SFT-type invariants.

In [18] (or [16]), I constructed families of contact manifolds $M$ in any odd dimension which have nonzero and finite algebraic torsion, in the sense of Latschev and Wendl [11]. In particular, they are tight and do not admit strong symplectic fillings. I also established, in higher dimensions, that Giroux torsion (in the sense of [16]) implies algebraic 1-torsion. My examples present a geometric structure which I call a spinal open book decomposition or SOBD (based on the 3-dimensional version of [11]). This geometric structure, which one could call partially planar, 'supports' a suitable contact structure and is of a certain type which can be 'detected' algebraically by the SFT machinery.

In this article, I discuss the same examples as in [18] from a 'dual' point of view. While the same underlying geometric decomposition is kept, the pieces of the decomposition have reversed roles. The resulting supported contact structure is isotopic to the original one, which is the expected behaviour, in terms of the expected 'Giroux'-type correspondence in the setting of SOBDs. However, the associated holomorphic data, originally a (2dimensional) finite-energy foliation, is replaced by a (2-codimensional) foliation by holomorphic hypersurfaces. While the holomorphic curve invariants should depend only on the isotopy class of the supported contact structure, the two points of view complement each other from a computational point of view. For instance, in [18] I used the finite-energy foliation to bound the order of algebraic torsion from above. In this article, I use the dual foliation to argue that the bounds from [18] are not, in general, necessarily the optimal ones.

After carrying out a detailed construction of examples in any odd dimension, I focus on a family of 5-dimensional particular cases in order to illustrate the techniques developed for the general case. For these, I study their SFT differential, up to order 2 . While my original aim was to show that the order of algebraic torsion is strictly greater than 1 , the result turned out to be quite unexpected. According to [16, Lemma 4.23] (which generalises [11, Lemma 4.15]), roughly speaking, if all configurations of holomorphic buildings with arithmetic genus $g$, no negative ends and $r$ positive ends satisfying $g+r \leq N+1$ cancel out in pairs when counted in SFT (after introducing an abstract polyfold perturbation), then there is no algebraic $N$-torsion. In the present case, after all possible such configurations for $N=1$ are classified (there are precisely 35 of them - see [16, Section 6.4]), all of them come in cancelling pairs except one, which I call sporadic. Then, algebraic 1-torsion might 
actually arise from differentiating the asymptotics of this configuration. While I cannot rigorously prove that algebraic 1-torsion indeed arises, I give a heuristic argument based on string topology [4] showing that it very likely does.

However, even though my computation is not the expected one, what is remarkable is that all the expected corollaries still hold. From knowledge of the SFT differential of my 5-dimensional examples, I show that they do not have Giroux torsion (which would follow if they did not have algebraic 1-torsion). I also obtain nonexistence results of symplectic cobordisms for these 5 -dimensional contact manifolds.

The failure of my computation can be given the following conjectural interpretation. Out of the 35 aforementioned configurations, there are two types: cylinders, and tori with one puncture. The former all come in cancelling pairs, and among the latter, only the sporadic one does not. For the corollaries, all I use is the cancellation phenomenon for cylinders, whereas the tori play no role whatsoever. In contrast, both are taken into consideration for algebraic 1-torsion. This suggests the existence of an invariant more subtle than algebraic torsion, which I suspect would be obtained from the rational SFT rather than the whole SFT, and still needs to be discovered. Morally, in the proof of the corollaries I would be exploiting the properties of this hypothetical invariant. I expect to pursue this line of research in further work.

A significant difficulty in practice is the lack of a higher-dimensional intersection theory between punctured holomorphic curves, in the sense of Richard Siefring. In dimensions 3 and 4, this is a good tool for proving that holomorphic curves with certain prescribed asymptotics are unique, and therefore one knows exactly what to count. In higher dimensions, although it does not make sense to count intersections between holomorphic curves, it does make sense to count intersections between holomorphic curves and hypersurfaces. But, as in the 4-dimensional situation, neither curves or hypersurfaces are closed, so that intersections coming 'from infinity' need to be considered.

In this article, I will obtain the first applications of the basic intersection theory between curves and hypersurfaces which are asymptotically cylindrical in a well-defined sense. This is outlined in [16, Appendix C], cowritten with Richard Siefring (to appear as an independent article [11]), and is a prequel to his upcoming work [18] generalising his results of [11]. I shall use the results of $[16,11]$ to restrict the behaviour of holomorphic curves in my examples. They necessarily lie in the leaves of a suitable codimension-2 holomorphic foliation. This will be crucial for extracting information from the SFT of my examples.

Moreover, we need to understand the number of ways certain holomorphic building configurations may glue to honest curves, which I intend to count, after making $J$ generic. For this, I make use of obstruction bundles in the sense of Hutchings and Taubes [11, 12], a fairly nontrivial gadget to deal with in practice. The idea is to count the number of honest curves obtained by gluing buildings, by algebraically counting the zero set of a section of an obstruction bundle. While I will not explicitly compute those numbers, and instead just prove existence results in suitable cases, the symmetries in the setup imply that there are cancellations which can be exploited to obtain my results. Under the assumption that obstruction bundles exist leafwise (which is satisfied for all applications of this article, since relevant curves are leafwise regular), I show their existence in my examples by 
careful analytical considerations, exploiting the fact that curves lies in hypersurfaces of the foliation.

\subsection{On the invariant}

The invariant I will use, algebraic torsion, was defined in [11] and is a contact invariant taking values in $\mathbb{Z}^{\geq 0} \cup\{\infty\}$. It was introduced, using the machinery of symplectic field theory, as a quantitative way of measuring nonfillability, giving rise to a 'hierarchy of fillability obstructions' (cf. [18]). At least morally, 0-torsion should correspond to overtwistedness, whereas 1-torsion is implied by Giroux torsion (the converse is not true). Having 0-torsion is actually equivalent to being algebraically overtwisted, which means that the contact homology, or equivalently its SFT, vanishes [11, Proposition 2.9]. This is well known to be implied by overtwistedness, but the converse is still wide open.

The key fact about this invariant is that it behaves well under exact symplectic cobordisms, which implies that the concave end inherits any order of algebraic torsion that the convex end has. Thus, algebraic torsion may be also thought of as an obstruction to the existence of exact symplectic cobordisms. In particular, finiteness of algebraic torsion is an obstruction to strong symplectic fillability (and in favourable cases, by choosing the right coefficients for the SFT, it obstructs certain weak fillings; see [16, Rem. 1.3, Cor. 1.5]). Moreover, there are connections to dynamics: any contact manifold with finite torsion satisfies the Weinstein conjecture (i.e., there exist closed Reeb orbits for every contact form).

It should be mentioned that there are other notions of algebraic torsion in the literature which do not use SFT, but which are only 3-dimensional (see [11] for the version using Heegaard Floer homology, or the appendix in [11] by Hutchings using Embedded Contact Homology $(\mathrm{ECH}))$.

\subsection{Statement of results}

For the SFT setup, I follow [11], to which I refer the reader for more details. I will take the SFT of a contact manifold $(M, \xi)$ (with coefficients) to be the homology $H_{*}^{S F T}(M, \xi ; \mathcal{R})$ of a $\mathbb{Z}_{2}$-graded unital $B V_{\infty}$-algebra $\left(\mathcal{A}[[\hbar]], \mathbf{D}_{S F T}\right)$ over the group $\operatorname{ring} R_{\mathcal{R}}:=\mathbb{R}\left[H_{2}(M ; \mathbb{R}) / \mathcal{R}\right]$, for some linear subspace $\mathcal{R} \subseteq H_{2}(M ; \mathbb{R})$. Here, $\mathcal{A}=\mathcal{A}(\lambda)$ has generators $q_{\gamma}$ for each good closed Reeb orbit $\gamma$ with respect to some nondegenerate contact form $\lambda$ for $\xi$, $\hbar$ is an even variable and the operator

$$
\mathbf{D}_{S F T}: \mathcal{A}[[\hbar]] \rightarrow \mathcal{A}[[\hbar]]
$$

is defined by counting rigid solutions to a suitable abstract perturbation of a $J$ holomorphic curve equation in the symplectisation of $(M, \xi)$. It satisfies

- $\mathbf{D}_{S F T}$ is odd and squares to zero,

- $\mathbf{D}_{S F T}(1)=0$ and

- $\mathbf{D}_{S F T}=\sum_{k \geq 1} D_{k} \hbar^{k-1}$, 
where $D_{k}: \mathcal{A} \rightarrow \mathcal{A}$ is a differential operator of order $\leq k$, given by

$$
D_{k}=\sum_{\substack{\Gamma^{+}, \Gamma^{-}, g, d \\\left|\Gamma^{+}\right|+g=k}} \frac{n_{g}\left(\Gamma^{+}, \Gamma^{-}, d\right)}{C\left(\Gamma^{-}, \Gamma^{+}\right)} q_{\gamma_{1}^{-}} \ldots q_{\gamma_{s^{-}}^{-}} z^{d} \frac{\partial}{\partial q_{\gamma_{1}^{+}}} \ldots \frac{\partial}{\partial q_{\gamma_{s^{+}}^{+}}} .
$$

The sum ranges over all nonnegative integers $g \geq 0$, homology classes $d \in H_{2}(M ; \mathbb{R}) / \mathcal{R}$ and ordered (possibly empty) collections of good closed Reeb orbits $\Gamma^{ \pm}=\left(\gamma_{1}^{ \pm}, \ldots, \gamma_{s^{ \pm}}^{ \pm}\right)$ such that $s^{+}+g=k$. After a choice of spanning surfaces as in [5, p. 566; see also p. 651], the projection to $M$ of each finite-energy holomorphic curve $u$ can be capped off to a 2-cycle in $M$, and so it gives rise to a homology class $[u] \in H_{2}(M)$, which I project to define $\overline{[u]} \in H_{2}(M ; \mathbb{R}) / \mathcal{R}$. The number $n_{g}\left(\Gamma^{+}, \Gamma^{-}, d\right) \in \mathbb{Q}$ denotes the count of (suitably perturbed) holomorphic curves of genus $g$ with positive asymptotics $\Gamma^{+}$and negative asymptotics $\Gamma^{-}$in the homology class $d$, including asymptotic markers as explained in $[5,21]$, and including rational weights arising from automorphisms. $C\left(\Gamma^{-}, \Gamma^{+}\right) \in \mathbb{N}$ is a combinatorial factor defined as $C\left(\Gamma^{-}, \Gamma^{+}\right)=s^{-} ! s^{+} ! \kappa_{\gamma_{1}^{-}} \ldots \kappa_{\gamma_{s^{-}}^{-}}$, where $\kappa_{\gamma}$ denotes the covering multiplicity of the Reeb orbit $\gamma$.

The most important special cases for my choice of linear subspace $\mathcal{R}$ are $\mathcal{R}=H_{2}(M ; \mathbb{R})$ and $\mathcal{R}=\{0\}$, called the untwisted and fully twisted cases, respectively, and $\mathcal{R}=\operatorname{ker} \Omega$ with $\Omega$ a closed 2 -form on $M$. I shall abbreviate the latter case as $H_{*}^{S F T}(M, \xi ; \Omega):=$ $H_{*}^{S F T}(M, \xi ; \operatorname{ker} \Omega)$, and the untwisted case as $H_{*}^{S F T}(M, \xi):=H_{*}^{S F T}\left(M, \xi ; H_{2}(M ; \mathbb{R})\right)$.

Definition 1.1. Let $(M, \xi)$ be a closed manifold of dimension $2 n+1$ with a positive, co-oriented contact structure. For any integer $k \geq 0$, we say that $(M, \xi)$ has $\Omega$-twisted algebraic torsion of order $k$ (or $\Omega$-twisted $k$-torsion) if $\left[\hbar^{k}\right]=0$ in $H_{*}^{S F T}(M, \xi ; \Omega)$. If this is true for all $\Omega$, or equivalently, if $\left[\hbar^{k}\right]=0$ in $H_{*}^{S F T}(M, \xi ;\{0\})$, then we say that $(M, \xi)$ has fully twisted algebraic $k$-torsion.

I will refer to untwisted $k$-torsion in the case where $\Omega=0$, in which case $\mathcal{R}=$ $H_{2}(M ; \mathbb{R}), R_{\mathcal{R}}=\mathbb{R}$, and we do not keep track of homology classes. Whenever I refer to torsion without mention of coefficients, I mean the untwisted version, and indeed in this article, as opposed to its predecessor [18], I will work only with the untwisted case (except when recalling Theorem 1.4). I will say that if a contact manifold has algebraic 0-torsion for every choice of coefficient ring, then it is algebraically overtwisted, which is equivalent to the vanishing of the SFT homology or its contact homology. By definition, $k$-torsion implies $(k+1)$-torsion, so we can define its algebraic torsion to be

$$
A T(M, \xi ; \mathcal{R}):=\min \left\{k \geq 0:\left[\hbar^{k}\right]=0\right\} \in \mathbb{Z}^{\geq 0} \cup\{\infty\},
$$

where we set $\min \emptyset=\infty$. Denote it by $A T(M, \xi)$ in the untwisted case.

Examples of 3-dimensional contact manifolds with any given order of torsion $k-1$, but not $k-2$, were constructed in [11]. I will consider a generalisation of their examples. Consider $\Sigma$ a surface of genus $g$ which is divided into two pieces $\Sigma_{+}$and $\Sigma_{-}$along some dividing set of simple closed curves $\Gamma$ of cardinality $k$, where $\Sigma_{-}$has genus 0 and $\Sigma_{+}$has genus $g-k+1$. Consider also a closed $(2 n-1)$-manifold $Y$ such that $Y \times[-1,1]$ admits the structure of a Liouville domain (which I call a cylindrical Liouville semifilling). Let $M_{g}:=Y \times \Sigma$. 


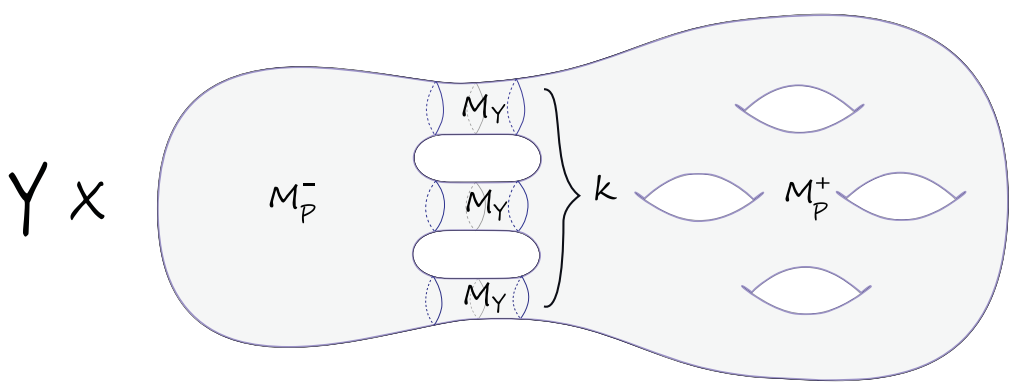

Figure 1. The SOBD structure in $M$.

Fix the following notation:

Notation 1.2. Throughout this document, the symbol $I$ will be reserved for the interval $[-1,1]$.

I can adapt the construction of the contact structures in [11] to my models. Decompose the manifold $M=M_{g}=Y \times \Sigma$ into three pieces

$$
M_{g}=M_{Y} \bigcup M_{P}^{ \pm}
$$

where $M_{Y}=\bigsqcup^{k} Y \times I \times S^{1}$ is the spine and $M_{P}^{ \pm}=Y \times \Sigma_{ \pm}$is the paper (see Figure 1). There is also a an extra distinguished region, denoted $M_{C}^{ \pm}$and called the interface, having two components which are smoothly of the form $\bigsqcup^{k} Y \times[-\delta, \delta] \times S^{1}$. It corresponds to the region where spine and paper glue together, and can be thought of as a smoothing of the corner $M_{Y} \cap M_{P}^{ \pm}$(see Figures 2 and 3; cf. Remark 3.3).

We have natural fibrations

$$
\begin{gathered}
\pi_{Y}: M_{Y} \rightarrow Y \times I \\
\pi_{P}^{ \pm}: M_{P}^{ \pm} \rightarrow Y_{ \pm},
\end{gathered}
$$

where $Y_{ \pm}:=Y \times\{ \pm 1\}$, with fibres $S^{1}$ and $\Sigma_{ \pm}$, respectively, and they are compatible in the sense that

$$
\partial\left(\left(\pi_{P}^{ \pm}\right)^{-1}(p t)\right)=\bigsqcup^{k} \pi_{Y}^{-1}(p t)
$$

where $p t \in Y_{ \pm} \subset Y \times I$. While $\pi_{Y}$ has a Liouville domain as base and a contact manifold as fibre, the situation is reversed for $\pi_{P}^{ \pm}$, which has a contact base and Liouville fibres. This is a prototypical example of an SOBD. While I will not include a formal definition of this notion, the reader is invited to consult [16] for a tentative one.

Using this decomposition, a contact structure $\xi_{k}$ can be constructed which is a small perturbation of the 'confoliation'-type stable Hamiltonian structure $\xi_{ \pm} \oplus T \Sigma_{ \pm}$along $M_{P}^{ \pm}$, and is a contactisation for the Liouville domain $(Y \times I, \epsilon d \alpha)$ along $M_{Y}$, for some small $\epsilon>0$. This means that it coincides with $\operatorname{ker}(\epsilon \alpha+d \theta)$, where $\theta$ is the $S^{1}$-coordinate. This was done in detail in [18], where the following was proved: 
Theorem 1.3 ([18]). For any $k \geq 1$ and $g \geq k$, the $(2 n+1)$-dimensional contact manifold $\left(M_{g}=Y \times \Sigma, \xi_{k}\right)$ satisfy $A T\left(M_{g}, \xi_{k}\right) \leq k-1$. In particular, it is not (strongly) symplectically fillable. Moreover, if $\left(Y, \alpha_{ \pm}\right)$are hypertight and $k \geq 2$, the corresponding contact manifold $\left(M_{g}, \xi_{k}\right)$ is also hypertight. In particular, $A T\left(M_{g}, \xi_{k}\right)>0$, and it is tight.

In this article, I consider the 'dual' SOBD, where the roles of the fibrations are reversed:

$$
\begin{gathered}
\pi_{Y}^{*}: M_{Y} \rightarrow S^{1} \\
\left(\pi_{P}^{ \pm}\right)^{*}: M_{P}^{ \pm} \rightarrow \Sigma_{ \pm},
\end{gathered}
$$

so that $\pi_{Y}^{*}$ is a Liouville fibration over a contact base and $\left(\pi_{P}^{ \pm}\right)^{*}$ a contact fibration over a Liouville base. Observe that we can do this due to the absence of monodromy (the fibrations are trivial).

The associated contact structure $\xi_{k}^{*}$ is now a perturbation of the (integrable) stable Hamiltonian structure $T(Y \times I)$ along $M_{Y}$, which is now the paper, and isotopic to a '( $\left.Y_{ \pm}, \alpha_{ \pm}\right)$-contactization' of the Liouville domain $\left(\Sigma_{ \pm}, \epsilon \lambda_{ \pm}\right)$along $M_{P}^{ \pm}$, which is now the spine (for some Liouville form $\lambda_{ \pm}$). More concretely, $\xi_{k}^{*}$ is isotopic, along $M_{P}^{ \pm}$, to $\operatorname{ker}\left(\epsilon \lambda_{ \pm}+\alpha_{ \pm}\right)$. One can check explicitly that $\xi_{k}$ and $\xi_{k}^{*}$ are isotopic contact structures [16]. I will drop the $*$ from the notation, since my statements depend only on the isotopy type of the contact structure. When needed, I will loosely refer to the original (undualised) SOBD and all the associated data (contact form, almost complex structure, foliation, etc.) as 'model A', and to the dualised SOBD and associated data as 'model B' (cf. Definition 3.1).

The authors of [16] define a generalised higher-dimensional version of the notion of Giroux torsion. This notion is defined as follows: consider $\left(\alpha_{+}, \alpha_{-}\right)$a Liouville pair on a closed manifold $Y^{2 n-1}$ - that is, the 1 -form $\beta=\frac{1}{2}\left(e^{s} \alpha_{+}+e^{-s} \alpha_{-}\right)$is Liouville in $\mathbb{R} \times Y$. Consider also the Giroux $2 \pi$-torsion domain modelled on $\left(Y, \alpha_{+}, \alpha_{-}\right)$given by the contact manifold $\left(G T, \xi_{G T}\right):=\left(Y \times[0,2 \pi] \times S^{1}, \operatorname{ker} \lambda_{G T}\right)$, where

$$
\lambda_{G T}=\frac{1+\cos (r)}{2} \alpha_{+}+\frac{1-\cos (r)}{2} \alpha_{-}+\sin (r) d \theta
$$

and the coordinates are $(r, \theta) \in[0,2 \pi] \times S^{1}$. Say that a contact manifold $\left(M^{2 n+1}, \xi\right)$ has Giroux torsion whenever it admits a contact embedding $i:\left(G T, \xi_{G T}\right) \hookrightarrow(M, \xi)$. In this situation, denote by $\mathcal{O}(G T) \subseteq H^{2}(M ; \mathbb{R})$ the annihilator of the subspace $i_{*}\left(H_{1}(Y ; \mathbb{R}) \otimes H_{1}\left(S^{1} ; \mathbb{R}\right)\right) \subset H_{2}(M ; \mathbb{R})$, where $i_{*}$ is the map induced by the embedding $i$ on $H_{2}$. The following was conjectured in [16] and proved in [18]:

Theorem 1.4 ([18]). If a contact manifold $\left(M^{2 n+1}, \xi\right)$ has Giroux torsion, then it has $\Omega$-twisted algebraic 1-torsion, for every $[\Omega] \in \mathcal{O}(G T)$, where GT is a Giroux $2 \pi$-torsion domain embedded in $M$.

Conversely, one could ask whether there exist examples of nonfillable contact manifolds without Giroux torsion. Examples of 3-dimensional weakly but not strongly fillable contact manifolds without Giroux torsion were given in [18]. In higher dimensions, the following theorem can be proved without appealing to the abstract perturbation scheme for SFT (see Disclaimer 1.11). I use the fact that the unit cotangent bundle of a closed hyperbolic surface fits into a cylindrical semifilling [11]. 
Theorem 1.5. Let $\left(M_{0}^{5}, \xi_{0}\right)$ be a 5-dimensional contact manifold with Giroux torsion, and let $Y$ be the unit cotangent bundle of a closed hyperbolic surface. If $\left(M=\Sigma \times Y, \xi_{k}\right)$ is the corresponding 5-dimensional contact manifold of Theorem 1.3 with $k \geq 3$, then there is no exact symplectic cobordism having $\left(M_{0}, \xi_{0}\right)$ as the convex end and $\left(M, \xi_{k}\right)$ as the concave end.

In particular, we obtain the following:

Corollary 1.6. If $Y$ is the unit cotangent bundle of a closed hyperbolic surface and $\left(M=\Sigma \times Y, \xi_{k}\right)$ is the corresponding 5-dimensional contact manifold of Theorem 1.3 with $k \geq 3$, then $\left(M, \xi_{k}\right)$ does not have Giroux torsion.

In the other direction of Theorem 1.4, examples of contact 3-manifolds which have 1torsion but not Giroux torsion were constructed in [11]. In higher dimensions, we have fairly strong geometric reasons for the following:

Conjecture 1.7. The examples of Corollary 1.6 have untwisted algebraic 1-torsion (for any $k \geq 1)$.

For $k \geq 3$, this would give an infinite family of contact manifolds with no Giroux torsion and algebraic 1-torsion in dimension 5 .

The interesting thing about this conjecture is that it builds on the relationship between SFT and string topology as discussed in [4]. There are certain nonzero counts of punctured tori in the symplectisation of ( $Y, \alpha)$, where $\alpha$ is the standard Liouville form, which survive in $M$. They are given in terms of the coefficients of the Goldman-Turaev cobracket operation on strings in the underlying hyperbolic surface. One can find elements in the SFT algebra of $(Y, \alpha)$ whose differential in $\mathbb{R} \times Y$ has nonzero contributions from these tori and other pair-of-pants configurations, but by index considerations only the former survive in $M$. This is how 1-torsion should arise. In fact, out of 35 possibilities, consisting of both cylinders and 1-punctured tori, this is the only possible configuration from which 1-torsion can arise (see Section 4.3). I will therefore refer to it as sporadic. However, I provide a heuristic argument as to why I expect it to be true.

Theorem 1.5 would then be a result which is beyond the scope of algebraic torsion, since the presence of such a cobordism would only yield the already-known fact that both ends have algebraic 1-torsion. In fact, in the proof of Theorem 1.5, only holomorphic cylinders play a role, whereas 1-punctured tori do not. In contrast, both are taken into consideration for 1-torsion. This suggests the existence of an invariant more subtle than algebraic torsion, which I suspect would be obtained from the rational SFT. Also, the computations in [11] (e.g., the proofs of Theorems 3 and 6 in that article) could be interpreted in this way.

Putting Theorem 1.3 together with Corollary 1.6, we obtain the following:

Corollary 1.8. There exist infinitely many nondiffeomorphic 5-dimensional contact manifolds $(M, \xi)$ which are tight and not strongly fillable and which do not have Giroux torsion.

To my knowledge, there are no other known examples of higher-dimensional contact manifolds as in Corollary 1.8 . 
The contact structure $\xi_{k}$ of Theorem 1.3 can be twisted close to the dividing set, by performing the l-fold Lutz-Mori twist along a hypersurface $H$ lying in $\partial\left(\bigsqcup^{k} Y \times I \times S^{1}\right)$. This notion was defined in [16], and builds on ideas by Mori in dimension 5 [21]. It consists of gluing copies, along $H$, of a $2 \pi l$-Giroux torsion domain $\left(G T_{l}, \xi_{G T}\right):=(Y \times$ $\left.[0,2 \pi l] \times S^{1}, \operatorname{ker} \lambda_{G T}\right)$, the contact manifold obtained by gluing $l$ copies of $G T=G T_{1}$ together. The resulting contact structures are all homotopic as almost contact structures. By construction, all of these have Giroux torsion, so by Theorem 1.4 they have $\Omega$-twisted 1-torsion, for $[\Omega] \in \mathcal{O}=\mathcal{O}(G T)$. See Appendix 4.6 for more details.

As a corollary of Theorem 1.5, we get the following:

Corollary 1.9. Let $Y$ be the unit cotangent bundle of a closed hyperbolic surface, and let $(M=\Sigma \times Y, \xi)$ be the corresponding 5-dimensional contact manifold of Theorem 1.3, with $k \geq 3$. If $\left(M, \xi_{l}\right)$ denotes the contact manifold obtained by an l-fold Lutz-Mori twist of $(M, \xi)$, then there is no exact symplectic cobordism having $\left(M, \xi_{l}\right)$ as the convex end and $(M, \xi)$ as the concave end (even though the underlying manifolds are diffeomorphic, and the contact structures are homotopic as almost contact structures).

In the proof of Theorem 1.5, I make use of obstruction bundles, in the sense of Hutchings and Taubes [11, 11]. I prove that they exist in my setup, under the condition that they exist inside the leaves of a codimension-2 holomorphic foliation. This suffices for all the applications of this article, since relevant curves are leafwise regular. As a by-product, I derive a result for superrigidity of holomorphic cylinders in 4-dimensional symplectic cobordisms, which might be of independent interest. This is a natural adaptation of the results of [11, Section 7] to the punctured setting. Recall that a somewhere injective holomorphic curve is superrigid if it is immersed and has index zero and the normal component of the linearised Cauchy-Riemann operator of every multiple cover is injective.

Theorem 1.10. For generic J, every somewhere injective holomorphic cylinder in a 4dimensional symplectic cobordism with index zero and vanishing Conley-Zehnder indices (in some trivialisation) is superrigid.

Disclaimer 1.11. Since the statements of my results make use of machinery from symplectic field theory, they come with the standard disclaimer that they assume that its analytic foundations are in place. However, I have taken special care in that the approach taken not only provides results that will be fully rigorous after the polyfold machinery of Hofer, Wysocki and Zehnder is complete but also gives several direct results that are already rigorous. Such is the case for Theorem 1.5, for example. Moreover, let me clarify that while it will not be necessary for my results here, polyfold counts and obstruction bundle counts (whenever these exist) are expected to agree.

\section{Guide to the article}

The main construction is carried out in Section 3, which corresponds to the building of my model contact manifolds and stable Hamiltonian structures in any odd dimension, from a 'dual' point of view as in [18]. I construct a foliation by holomorphic hypersurfaces in Section 3.4. In Section 3.6, I use the results from [11] to show that relevant holomorphic 
curves lie in the leaves of the foliation. I discuss obstruction bundles in Section 3.8 from a fairly general point of view, and I prove their existence for curves in the symplectisation of my models, assuming their existence leafwise.

I illustrate my techniques in Section 4.3, where I study the algebraic 1-torsion of a special family of contact manifolds in dimension 5. I discuss the sporadic configuration in Section 4.4, and its relationship to string topology. Theorem 1.5 is proved in Section 4.5.

In Appendix 4.6 I derive Theorem 1.10. In Appendix 4.6 I describe the Lutz-Mori twists and apply them to my model contact manifolds to obtain the contact structures of Corollary 1.9.

\subsection{Basic notions}

A contact form in a $(2 n+1)$-dimensional manifold $M$ is a 1 -form $\alpha$ such that $\alpha \wedge d \alpha^{n}$ is a volume form, and the associated contact structure is $\xi=\operatorname{ker} \alpha$ (I will assume that all the contact structures are co-oriented). The Reeb vector field associated to $\alpha$ is the unique vector field $R_{\alpha}$ on $M$ satisfying

$$
\alpha\left(R_{\alpha}\right)=1, i_{R_{\alpha}} d \alpha=0 .
$$

A $T$-periodic Reeb orbit is $(\gamma, T)$, where $\gamma: \mathbb{R} \rightarrow M$ is such that $\dot{\gamma}(t)=T R_{\alpha}(\gamma(t))$, $\gamma(1)=\gamma(0)$. I will often just talk about a Reeb orbit $\gamma$ without mention of $T$, called its period, or action. If $\tau>0$ is the minimal number for which $\gamma(\tau)=\gamma(0)$, and $k \in \mathbb{Z}^{+}$ is such that $T=k \tau$, we say that the covering multiplicity of $(\gamma, T)$ is $k$. If $k=1$, then $\gamma$ is said to be simply covered (otherwise it is multiply covered). A periodic orbit $\gamma$ is said to be nondegenerate if the restriction of the time $T$ linearised Reeb flow $d \varphi^{T}$ to $\xi_{\gamma(0)}$ does not have 1 as an eigenvalue. More generally, a Morse-Bott submanifold of $T$-periodic Reeb orbits is a closed submanifold $N \subseteq M$ invariant under $\varphi^{T}$ such that $\operatorname{ker}\left(d \varphi^{T}-\mathbb{1}\right)=T N$, and $\gamma$ is Morse-Bott whenever it lies in a Morse-Bott submanifold and its minimal period agrees with the nearby orbits in the submanifold. The vector field $R_{\alpha}$ is nondegenerate/Morse-Bott if all of its closed orbits are nondegenerate/Morse-Bott.

A stable Hamiltonian structure (SHS) on $M$ is a pair $\mathcal{H}=(\Lambda, \Omega)$ consisting of a closed 2 -form $\Omega$ and a 1 -form $\Lambda$ such that

$$
\operatorname{ker} \Omega \subseteq \operatorname{ker} d \Lambda \text {, and }\left.\Omega\right|_{\xi} \text { is nondegenerate, where } \xi=\operatorname{ker} \Lambda .
$$

In particular, $(\alpha, d \alpha)$ is an SHS whenever $\alpha$ is a contact form. The Reeb vector field associated to $\mathcal{H}$ is the unique vector field on $M$ defined by

$$
\Lambda(R)=1, i_{R} \Omega=0 .
$$

There are analogous notions of nondegeneracy/Morse-Bottness for SHSs.

A symplectic form in a $2 n$-dimensional manifold $W$ is a 2 -form $\omega$ which is closed and nondegenerate. A Liouville domain is a compact symplectic manifold with an exact symplectic form $\omega=d \lambda$, such that the associated Liouville vector field $V$ (defined by the equation $i_{V} d \lambda=\lambda$ ) is transverse to the boundary. Any Liouville domain necessarily has nonempty boundary. A boundary component $M$ of a Liouville domain (endowed with the boundary orientation) is convex if the Liouville vector field is positively transverse to $M$, and is concave if it is so negatively. An exact cobordism from a (co-oriented) 
contact manifold $\left(M_{+}, \xi_{+}\right)$to $\left(M_{-}, \xi_{-}\right)$is a Liouville domain $(W, \omega=d \alpha)$ with boundary $\partial W=M_{+} \bigsqcup M_{-}$, where $M_{+}$is convex, $M_{-}$is concave and $\left.\operatorname{ker} \alpha\right|_{M_{ \pm}}=\xi_{ \pm}$. Therefore, the boundary orientation induced by $\omega$ agrees with the contact orientation on $M_{+}$and differs on $M_{-}$. A Liouville filling of a (possibly disconnected) contact manifold $(M, \xi)$ is a compact Liouville cobordism from $(M, \xi)$ to the empty set. A strong symplectic cobordism and a strong filling are defined in the same way, with the difference that $\omega$ is exact only in a neighbourhood of the boundary of $W$ (so that the Liouville vector field is defined in this neighbourhood but not necessarily in its complement). A contact manifold $\left(M^{2 n-1}, \xi\right)$ is weakly fillable if it is the smooth boundary of a symplectic $2 n$-manifold $\left(W^{2 n}, \omega\right)$, such that $\left.(\omega+\tau d \lambda)\right|_{\xi}$ is symplectic for every $\tau \geq 0$, for one (and hence every) choice of contact form $\lambda$. Say then that $(W, \omega)$ is a weak filling for $(M, \xi)$.

The symplectisation of a contact manifold $(M, \xi=\operatorname{ker} \alpha)$ is the symplectic manifold $\left(\mathbb{R} \times M, \omega=d\left(e^{a} \alpha\right)\right.$ ), where $a$ is the $\mathbb{R}$-coordinate. A Liouville manifold is a noncompact symplectic manifold which is obtained from a Liouville domain by attaching cylindrical ends at each boundary component, modelled on (half-)symplectisations of the contact structures at the corresponding boundary component. In particular, a symplectisation is a Liouville manifold. Similarly, the symplectisation of a stable Hamiltonian manifold $(M, \Lambda, \Omega)$ is the symplectic manifold $\left(\mathbb{R} \times M, \omega^{\varphi}\right)$, where $\omega^{\varphi}=d(\varphi(a) \Lambda)+\Omega$ and $\varphi$ is an element of the set

$$
\mathcal{P}=\left\{\varphi \in C^{\infty}(\mathbb{R},(-\epsilon, \epsilon)): \varphi^{\prime}>0\right\} .
$$

Here, $\epsilon>0$ is chosen small enough so that $\omega^{\varphi}$ is indeed symplectic. An $\mathcal{H}$-compatible (or simply cylindrical) almost complex structure on a symplectisation $\left(W=\mathbb{R} \times M, \omega^{\varphi}\right)$ is $J \in \operatorname{End}(T W)$ such that

$$
J \text { is } \mathbb{R} \text {-invariant, } J^{2}=-\mathbb{1}, J\left(\partial_{a}\right)=R, J(\xi)=\xi,\left.J\right|_{\xi} \text { is } \Omega \text {-compatible. }
$$

The last condition means that $\Omega(\cdot, J \cdot)$ defines a $J$-invariant Riemannian metric on $\xi$. If $J$ is $\mathcal{H}$-compatible, then it is easy to check that it is $\omega^{\varphi}$-compatible, which means that $\omega^{\varphi}(\cdot, J \cdot)$ is a $J$-invariant Riemannian metric on $\mathbb{R} \times M$.

I will consider, for cylindrical $J$, punctured $J$-holomorphic curves $u:(\dot{\Sigma}, j) \rightarrow(\mathbb{R} \times M, J)$ in the symplectisation of a stable Hamiltonian manifold $M$, where $\dot{\Sigma}=\Sigma \backslash \Gamma$ with $\Gamma$ a finite set of punctures, $(\Sigma, j)$ is a compact connected Riemann surface and $u$ satisfies the nonlinear Cauchy-Riemann equation $d u \circ j=J \circ u$. I will also assume that $u$ is asymptotically cylindrical, which means the following. Partition the punctures into positive and negative subsets $\Gamma=\Gamma^{+} \cup \Gamma^{-}$, and at each $z \in \Gamma^{ \pm}$, choose a biholomorphic identification of a punctured neighbourhood of $z$ with the half-cylinder $Z_{ \pm}$, where $Z_{+}=[0, \infty) \times S^{1}$ and $Z_{-}=(-\infty, 0] \times S^{1}$. Then writing $u$ near the puncture in cylindrical coordinates $(s, t)$, for $|s|$ sufficiently large, it satisfies an asymptotic formula of the form

$$
u \circ \phi(s, t)=\exp _{(T s, \gamma(T t))} h(s, t) .
$$

Here $T>0$ is a constant, $\gamma: \mathbb{R} \rightarrow M$ is a $T$-periodic Reeb orbit, the exponential map is defined with respect to any $\mathbb{R}$-invariant metric on $\mathbb{R} \times M, h(s, t) \in \xi_{\gamma(T t)}$ goes to 0 uniformly in $t$ as $s \rightarrow \pm \infty$ and $\phi: Z_{ \pm} \rightarrow Z_{ \pm}$is a smooth embedding such that $\phi(s, t)-$ 
$\left(s+s_{0}, t+t_{0}\right) \rightarrow 0$ as $s \rightarrow \pm \infty$ for some constants $s_{0} \in \mathbb{R}, t_{0} \in S^{1}$. I will refer to punctured asymptotically cylindrical $J$-holomorphic curves simply as $J$-holomorphic curves.

Observe that for any closed Reeb orbit $\gamma$ and cylindrical $J$, the trivial cylinder over $\gamma$, defined as $\mathbb{R} \times \gamma$, is $J$-holomorphic.

The Fredholm index of a punctured holomorphic curve $u$ which is asymptotic to nondegenerate Reeb orbits in a $(2 n+2)$-dimensional symplectisation $W^{2 n+2}=\mathbb{R} \times M$ is given by the formula

$$
\operatorname{ind}(u)=(n-2) \chi(\dot{\Sigma})+2 c_{1}^{\tau}\left(u^{*} T W\right)+\mu_{C Z}^{\tau}(u) .
$$

Here, $\dot{\Sigma}$ is the domain of $u$ and $\tau$ denotes a choice of trivialisations for each of the bundles $\gamma_{z}^{*} \xi$, where $z \in \Gamma$, at which $u$ approximates the Reeb orbit $\gamma_{z}$. The term $c_{1}^{\tau}\left(u^{*} T W\right)$ is the relative first Chern number of the bundle $u^{*} T W$. In the case where $W$ is 2-dimensional, this is defined as the algebraic count of zeroes of a generic section of $u^{*} T W$ which is asymptotically constant with respect to $\tau$. For higher-rank bundles, $c_{1}^{\tau}$ is determined by imposing the condition that it is invariant under bundle isomorphisms and satisfies the Whitney sum formula (see, e.g., [21]). The term $\mu_{C Z}^{\tau}(u)$ is the total Conley-Zehnder index of $u$, given by

$$
\mu_{C Z}^{\tau}(u)=\sum_{z \in \Gamma^{+}} \mu_{C Z}^{\tau}\left(\gamma_{z}\right)-\sum_{z \in \Gamma^{-}} \mu_{C Z}^{\tau}\left(\gamma_{z}\right) .
$$

\section{Model contact manifolds: A dual point of view}

I will construct a contact model for the underlying manifold $M=Y \times \Sigma$. Here, as described in the introduction, $Y$ fits into a cylindrical semifilling and $\Sigma$ is an orientable genus $g$ surface, decomposed into a genus zero piece $\Sigma_{-}$and a positive genus piece $\Sigma_{+}$ along a dividing set of $k$ circles. The main features of this contact model are as follows: closed Reeb orbits of low action correspond to pairs $(p, \gamma)$ of critical points $p$ of suitable Morse functions on $\Sigma_{ \pm}$and closed $R_{ \pm}$-orbits $\gamma$ in $\left(Y, \alpha_{ \pm}\right)$; and we will have a foliation of its symplectisation by holomorphic hypersurfaces for a suitable almost complex structure compatible with an SHS deforming the contact data. These hypersurfaces project to flow lines on the surface $\Sigma$, and they can be identified with either cylindrical completions of the Liouville domain $Y \times I$ or symplectisations of its boundary components. The results in [11] can then be used to prove that any holomorphic curves necessarily lie in a hypersurface of this foliation, which is the key tool for restricting their behaviour. After constructing the model, I will restrict my attention to a specific family of 5-dimensional models for which I prove Theorem 1.5.

\subsection{Construction of the model contact manifolds}

Consider $Y^{2 n-1}$ such that $(Y \times I, d \alpha)$ is a cylindrical Liouville semifilling. Assume that $\alpha=\left\{\alpha_{r}\right\}_{r \in I}$ is a 1-parameter family of 1-forms such that $\alpha_{r}$ is contact for $r \neq 0$. All known examples of such semifillings admit an $\alpha$ satisfying this condition, and I will make it a standing assumption (which is by no means crucial). I will denote by $\xi_{ \pm}=\operatorname{ker} \alpha_{ \pm}$the contact structures on $Y_{ \pm}:=Y \times\{ \pm 1\}$ where $\alpha_{ \pm}=\alpha_{ \pm 1}=\left.\alpha\right|_{Y_{ \pm}}$, by $V$ the Liouville vector 
field associated to $d \alpha$ and by $R_{r}$ the Reeb vector field associated to $\alpha_{r}$ for $r \neq 0$, and $R_{ \pm}:=R_{ \pm 1}$. Consider also $\Sigma=\Sigma_{-} \cup_{\partial} \Sigma_{+}$, a genus $g$ surface obtained by gluing a genus zero surface $\Sigma_{-}$to a genus $g-k+1>0$ surface $\Sigma_{+}$, along $k$ boundary components in an orientation-reversing manner, and the manifold $M=Y \times \Sigma$. Throughout this article, I shall use the convention that whenever I deal with equations involving \pm or $\mp$, they are interpreted as having a different sign according to the region (the upper sign denotes the 'plus' region, and the lower, the 'minus' region).

Take collar neighbourhoods of each boundary component of $\Sigma_{ \pm}$of the form $\mathcal{N}\left(\partial \Sigma_{ \pm}\right):=$ $\bigsqcup^{k}[-1,0] \times S^{1}$, with coordinates $\left(t_{ \pm}, \theta\right) \in[-1,0] \times S^{1}$, such that $\partial \Sigma_{ \pm}=\left\{t_{ \pm}=0\right\}$. Glue these surfaces together to a cylindrical region $\mathcal{U}$ having $k$ components, each of which looks like $[-1,1] \times S^{1} \ni(r, \theta)$, so that $r=\mp t_{ \pm}$. I now use the fact that each surface $\Sigma_{ \pm}$ carries a Stein structure providing a Stein filling of its 1-dimensional convex contact boundary. That is, we can take Morse functions $h_{ \pm}$on $\Sigma_{ \pm}$which are plurisubharmonic with respect to a suitable complex structure $j_{ \pm}$, which in the collar neighbourhoods look like $h_{ \pm}\left(t_{ \pm}, \theta\right)=e^{t_{ \pm}}$and $j_{ \pm}\left(\partial_{t_{ \pm}}\right)=\partial_{\theta}$. Moreover, we have that $\lambda_{ \pm}=d^{\mathbb{C}} h_{ \pm}=-d h_{ \pm} \circ j_{ \pm}$is a Liouville form, with a Liouville vector field given by $\nabla h_{ \pm}$, where the gradient is computed with respect to the metric $g_{ \pm}=g_{d \lambda_{ \pm}, j_{ \pm}}=d \lambda_{ \pm}\left(\cdot, j_{ \pm} \cdot\right)$ and where we can assume that the gradient flow for $h_{ \pm}$is Morse-Smale (see [11, Lemma 4.1] for details). Thus, in the collar neighbourhoods we have $\lambda_{ \pm}=e^{t_{ \pm}} d \theta=e^{\mp r} d \theta$ and $\nabla h_{ \pm}=\partial_{t_{ \pm}}=\mp \partial_{r}$. The Hamiltonian vector field $X_{h_{ \pm}}=j_{ \pm} \nabla h_{ \pm}$, computed with respect to the symplectic form $d \lambda_{ \pm}$inducing the orientation in $\Sigma_{ \pm}$, is tangent to the contact-type level sets.

Take the orientation on $M=Y \times \Sigma$ to be the one induced by $\alpha_{-}$on the first factor, and on the second factor orient $\Sigma$ so that its piece $\Sigma_{-}$is oriented by $d \lambda_{-}$and its piece $\Sigma_{+}$is oriented by the opposite orientation, given by $d \lambda_{+}$. Take both $h_{ \pm}$to have a unique minimum in $\Sigma_{ \pm}$; I will use the convention of calling the minimum of $h_{+}$the maximum (cf. Remark 3.9).

Define for $K_{+}>K_{-} \gg 0$ large constants a smooth function $g_{0}:[-1,1] \rightarrow \mathbb{R}$, such that it is constant equal to $K_{-}$in $[-1,-1+\delta)$ for some small $\delta>0$, equal to $K_{+}$in $(1-\delta, 1], g_{0}>0$ everywhere and $g_{0}^{\prime} \geq 0$ with strict inequality in the interval $(-1+\delta, 1-\delta)$. The parameter $\delta>0$ is chosen so that the Liouville vector field in $Y \times[-1,1]$ is given by $V= \pm \partial_{r}$ in the corresponding components of $\{|r|>1-\delta\}$. Define also a function $\gamma:[-1,1] \rightarrow \mathbb{R}$ so that $\gamma(r)=-e^{\mp r}$ in $\{|r|>1-\delta\}$, and $\gamma<0$. Take

$$
g_{\epsilon}(r)=g_{0}(r)+\epsilon^{2} \gamma(r)
$$

where $\epsilon$ is chosen small enough so that $g_{\epsilon}>0$.

Now define

$$
h_{\epsilon}= \begin{cases}K_{ \pm}-\epsilon^{2} h_{ \pm}, & \text {in } \Sigma \backslash \mathcal{U} \\ g_{\epsilon}, & \text { in } \mathcal{U} .\end{cases}
$$

This is a (well-defined and smooth) function on $\Sigma$, and $\epsilon$ can be chosen small enough so that $h_{\epsilon}>0$.

Next, choose a smooth function $\psi:[-1,1] \rightarrow \mathbb{R}^{+}$satisfying $\psi(r)=e^{\mp r}$ in $\{|r|>1-\delta / 3\}$, $\psi \equiv 1$ on $[-1+\delta, 1-\delta], r \psi^{\prime}(r) \leq 0$. Define

$$
f_{\epsilon}(r)=\epsilon \psi(r)
$$


so that $f_{\epsilon}>0, f_{\epsilon}^{\prime} \equiv 0$ along $[-1+\delta, 1-\delta]$.

With these choices, let $\Lambda_{\epsilon}$ be the 1-parameter family of 1-forms in $M=Y \times \Sigma$ given by

$$
\Lambda_{\epsilon}= \begin{cases}\epsilon \lambda_{ \pm}+h_{\epsilon} \alpha_{ \pm}, & \text {in } Y \times(\Sigma \backslash \mathcal{U}) \\ f_{\epsilon} d \theta+h_{\epsilon} \alpha, & \text { in } Y \times \mathcal{U} .\end{cases}
$$

We can slightly modify $\alpha$ so that the different expressions in $\Lambda_{\epsilon}$ glue together smoothly: we have $\alpha=e^{ \pm r-1} \alpha_{ \pm}$in the corresponding components of the region $\{|r| \geq 1-\delta\}$ ), and we can replace $\alpha$ by a 1 -form (of the same name) which looks like $\phi(r) \alpha_{ \pm}$. Here, $\phi:\{|r| \geq 1-\delta\} \rightarrow \mathbb{R}$ is a smooth function which coincides with $e^{ \pm r-1}$ near $|r|=1-\delta$, equals 1 on $|r|=1$ and has a nonnegative/nonpositive derivative in the positive/negative components of $\{|r| \geq 1-\delta\}$. I shall denote $\xi_{\epsilon}:=\operatorname{ker} \Lambda_{\epsilon}$ (this was called $\xi_{k}$ in the introduction).

Definition 3.1. I shall refer to the family of contact forms $\Lambda_{\epsilon}$ (see Lemma 3.2) as model $B$, in contrast with model $A$, which is the family of contact forms for $M$ constructed in $[18]$.

\section{Lemma 3.2.}

1. For a fixed $K_{+} \gg 0, \Lambda_{\epsilon}$ is a contact form for $K_{-}$sufficiently close to $K_{+}$, and sufficiently small $\epsilon>0$.

2. Model $A$ is isotopic to model B.

Proof: I will prove this lemma by viewing model $\mathrm{B}$ as arising from the double completion construction (see $[11,16]$ ). This is something very useful which can be used to produce a suitable stable Hamiltonian structure deforming the contact data, as well as the required isotopy between both models. Morally, both models should be isotopic, since they are 'supported' by the same geometric decomposition underlying the dual SOBDs.

Denote by $\mathcal{N}^{\infty}(Y)=Y \times(1-\delta,+\infty) \times S^{1}$ and $\mathcal{N}^{\infty}(-Y)=Y \times(-\infty,-1+\delta) \times S^{1}$. Also, denote by $\Sigma_{ \pm}^{\infty}$ the Liouville completion of $\Sigma_{ \pm}$obtained by attaching cylindrical ends of the form $(-\delta,+\infty) \times S^{1}$ at each boundary component. The coordinates $t_{ \pm}$and $\theta$ extend to the cylindrical ends in an obvious way; denote the latter by $\mathcal{N}\left(\partial \Sigma_{ \pm}^{\infty}\right)$. We also have

$$
\begin{gathered}
M_{P}^{ \pm, \infty}=Y \times \Sigma_{ \pm}^{\infty} \\
M_{Y}^{\infty}=Y \times \mathbb{R} \times S^{1} .
\end{gathered}
$$

Take a variable $r$ parametrising the $\mathbb{R}$-direction in the completion $Y \times \mathbb{R}$. Now define the double completion as the open manifold

$$
E^{\infty, \infty}=(-\infty,-1+\delta) \times M_{P}^{-, \infty} \bigsqcup(1-\delta,+\infty) \times M_{P}^{+, \infty} \bigsqcup(-1,+\infty) \times M_{Y}^{\infty} / \sim,
$$

where we identify $\left(r, y, t_{-}, \theta\right) \in(-\infty,-1+\delta) \times Y \times \mathcal{N}\left(\partial \Sigma_{-}^{\infty}\right)$ with $(t, y, r, \theta) \in(-1,+$ $\infty) \times \mathcal{N}^{\infty}(-Y)$ if and only if $t=t_{-}$, and $\left(r, y, t_{+}, \theta\right) \in(1-\delta,+\infty) \times Y \times \mathcal{N}\left(\partial \Sigma_{+}^{\infty}\right)$ with $(t, y, r, \theta) \in(-1,+\infty) \times \mathcal{N}^{\infty}(Y)$ if and only if $t=t_{+}$. Denote by $E^{\infty, \infty}(t)$ the subset of $E^{\infty, \infty}$ where the variable $t$ is defined (see Figure 2). 


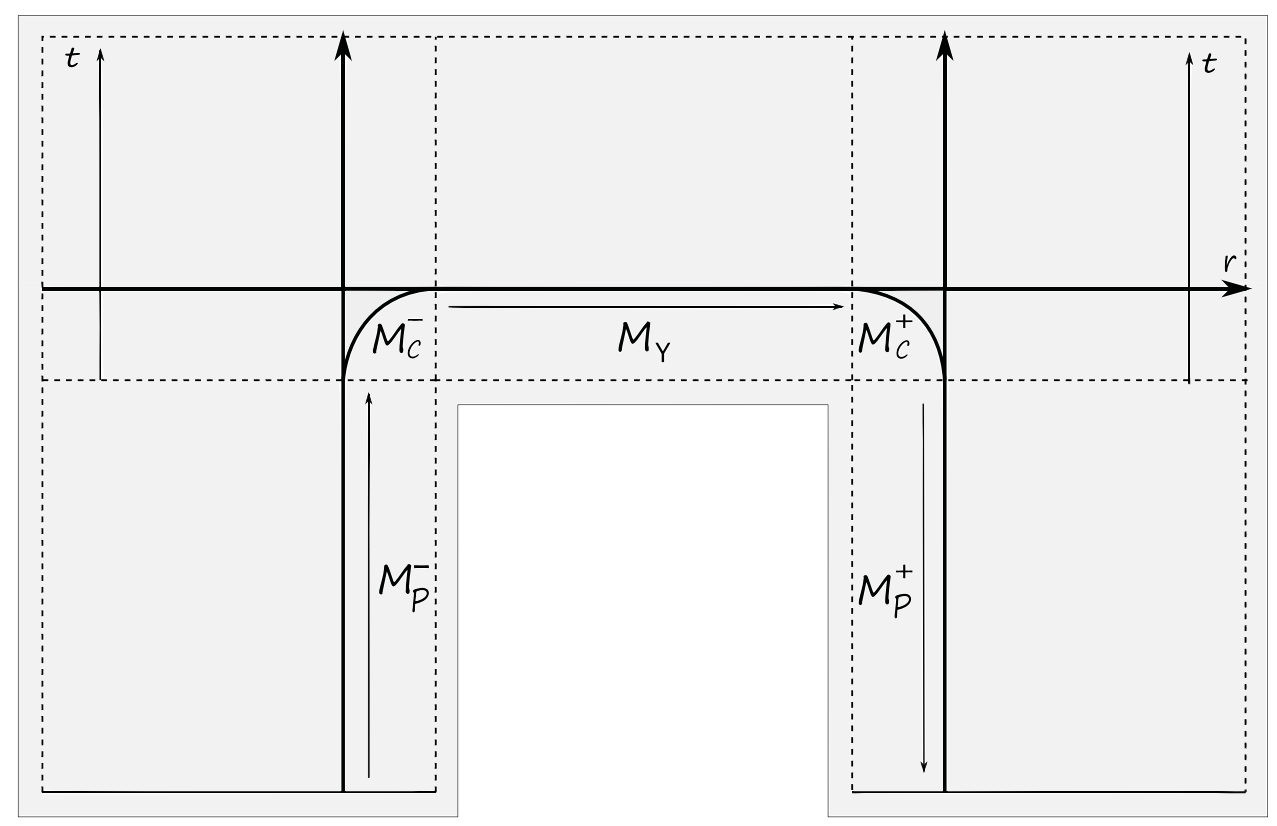

Figure 2. The double completion $E^{\infty, \infty}$.

Choose smoothing functions $F_{+}, G_{+}:(1-\delta, 1) \rightarrow \mathbb{R}$ and $F_{-}, G_{-}:(-1,-1+\delta) \rightarrow \mathbb{R}$ (see Figure 3) satisfying

$$
\begin{cases}\left(F_{+}(\rho), G_{+}(\rho)\right)=(\rho-1,0), & \text { for } 1-\delta<\rho \leq 1-2 \delta / 3 \\ \left(F_{-}(\rho), G_{-}(\rho)\right)=(0, \rho), & \text { for }-1<\rho \leq-1+\delta / 3 \\ G_{+}^{\prime}(\rho)<0, F_{+}^{\prime}(\rho)>0, & \text { for } 1-2 \delta / 3<\rho<1-\delta / 3 \\ F_{-}^{\prime}(\rho)>0, G_{-}^{\prime}(\rho)>0, & \text { for }-1+\delta / 3<\rho<-1+2 \delta / 3 \\ \left(F_{+}(\rho), G_{+}(\rho)\right)=(0,-\rho), & \text { for } 1-\delta / 3 \leq \rho<1 \\ \left(F_{-}(\rho), G_{-}(\rho)\right)=(\rho+1,0), & \text { for }-1+2 \delta / 3 \leq \rho<-1+\delta .\end{cases}
$$

For $L \geq 1$ and $Q \geq 0$, define $M^{L, Q}$ as the codimension- 1 submanifold of $E^{\infty, \infty}$ given by

$$
M^{L, Q}:=\left\{\partial E^{L, Q} \cap(\{r \in[-L+\delta, L-\delta]\} \cup\{t \leq Q-1\})\right\} \bigcup M_{C}^{ \pm, L, Q},
$$

where $E^{L, Q}=E^{\infty, \infty} \backslash(\{|r|>L\} \cup\{t>Q\})$, and

$$
\begin{aligned}
M_{C}^{ \pm, L, Q}= & \left\{\left(r=F_{ \pm}(\rho) \pm L, t=G_{ \pm}(\rho)+Q, y, \theta\right):\right. \\
& \left.(\rho, y, \theta) \in\left(\mathbb{R}^{ \pm} \cap\{1>|\rho|>1-\delta\}\right) \times Y \times S^{1}\right\}
\end{aligned}
$$

are the smoothings of the corners of $E^{L, Q}$.

We then have a decomposition

$$
M^{L, Q}=M_{P}^{ \pm, L, Q} \bigcup M_{C}^{ \pm, L, Q} \bigcup M_{Y}^{L, Q}
$$

which we define as

$$
M_{P}^{ \pm, L, Q}:=\left(Y \times\left(\Sigma_{ \pm} \backslash \mathcal{U}\right)\right) \cap M^{L, Q}
$$



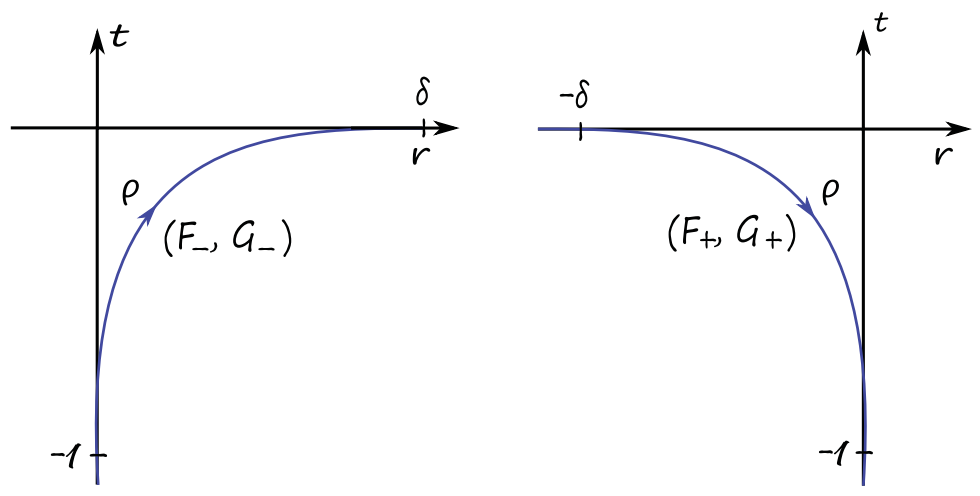

Figure 3. The choice of smoothing.

$$
\left.M_{Y}^{L, Q}:=\left(Y \times[-1+\delta, 1-\delta] \times S^{1}\right)\right) \cap M^{L, Q} .
$$

Remark 3.3. I call $M_{P}^{ \pm, L, Q}$ the spine, the region $M_{Y}^{L, Q}$ the paper (these regions should have swapped names and be called paper and spine, respectively, for the model A version) and $M_{C}^{ \pm, L, Q}$ the interface or smoothed corner. With this notation, $\mathcal{U}=M_{Y}^{L, Q} \cup M_{C}^{ \pm, L, Q}$. This is a spinal open book decomposition for $M^{L, Q}$. I shall drop the $L, Q$ from the notation when talking about $L=1, Q=0$. Note that $M^{L, Q}$ is diffeomorphic to $M$ for every $L, Q$.

If we define

$$
\widehat{E}^{\infty, \infty}=\bigcup_{L \geq 1} M^{L, L-1} \subset E^{\infty, \infty},
$$

which is foliated by the disjoint submanifolds $M^{L, L-1}$, the function $h_{\epsilon}: M \rightarrow \mathbb{R}$ can be extended to $\widehat{E}^{\infty, \infty}$ by fixing suitable identifications of $M^{L, L-1}$ with the diffeomorphic manifold $M=M^{1,0}$ via the following:

- $h_{\epsilon}(y, z, r):=h_{\epsilon}(z)$, for $(y, z, r) \in \widehat{E}^{\infty, \infty} \cap E^{\infty, \infty}(t)^{c}=Y \times\left(\Sigma_{ \pm} \backslash \mathcal{N}\left(\partial \Sigma_{ \pm}\right)\right) \times\{|r| \geq 1\}$.

- $h_{\epsilon}\left(F_{ \pm}(\rho) \pm L, G_{ \pm}(\rho)+L-1, y, \theta\right):=h_{\epsilon}(r)$, for $\left(F_{ \pm}(\rho) \pm L, G_{ \pm}(\rho)+L-1, y, \theta\right) \in M_{C}^{ \pm, L, L-1}$.

- $h_{\epsilon}(y, r, t, \theta):=h_{\epsilon}\left(\frac{1-\delta}{L-\delta} r\right)$, for $(y, r, t, \theta) \in Y \times[-L+\delta, L-\delta] \times S^{1} \subseteq M_{Y}^{L, L-1}$.

- $h_{\epsilon}(y, \pm L, t, \theta) \equiv h_{\epsilon}( \pm 1)$, for $(y, \pm L, t, \theta) \in M_{P}^{ \pm, L, L-1} \cap E^{\infty, \infty}(t)$.

Also extend $\lambda_{ \pm}$and $\alpha$ to $E^{\infty, \infty}$, so that they look like $\lambda=e^{t} d \theta$ and $\alpha=e^{\mp r-1} \alpha_{ \pm}$over the cylindrical ends. I claim that the 1 -form

$$
\Lambda_{\epsilon}:=\epsilon \lambda+h_{\epsilon} \alpha
$$

can be made Liouville in $E^{\infty, \infty}$ after sufficiently shrinking the first derivative of $h_{\epsilon}$ (in the uniform norm) but keeping $h_{\epsilon}$ uniformly large. By a version of the Thurston trick [16], we need only check that $h_{\epsilon} \alpha$ can be made Liouville in $Y \times \mathbb{R} \subset\{t=0\}$, over which $E^{\infty, \infty}$ is a 'fibration' with symplectic fibres with cylindrical ends (which change topology). 
Let $d v o l$ be the volume form in $Y$ with respect to the $\alpha_{-}$-orientation, defined by the equation

$$
d \alpha^{n}=d v o l \wedge d r
$$

We can then write

$$
\alpha_{r} \wedge d \alpha_{r}^{n-1}=\operatorname{link}\left(\alpha_{r}\right) d v o l,
$$

where $r \mapsto \operatorname{link}\left(\alpha_{r}\right) \in C^{\infty}(Y)$ is the self-linking function, whose sign is opposite to that of $r \in \mathbb{R}$. We then have

$$
d\left(h_{\epsilon} \alpha\right)^{n}=h_{\epsilon}^{n-1}\left(h_{\epsilon}-n h_{\epsilon}^{\prime} \operatorname{link}\left(\alpha_{r}\right)\right) d v o l \wedge d r .
$$

Observe that even though

$$
\operatorname{link}\left(\alpha_{r}\right)=e^{n( \pm r-1)} \operatorname{link}\left(\alpha_{ \pm}\right)
$$

over the cylindrical ends, and hence is not bounded as a function of $r$ on the whole real line, our choice of extension of $h_{\epsilon}$ implies that $h_{\epsilon}^{\prime} \equiv 0$ for $|r| \geq 2$. We can choose $h_{\epsilon}^{\prime}$ uniformly small enough on $[-2,2]$, without making $h_{\epsilon}$ small, by observing that $h_{\epsilon}^{\prime}=g_{\epsilon}^{\prime}=g_{0}^{\prime}+\epsilon^{2} \gamma^{\prime}$ can be made small by taking $\epsilon$ small and $K_{-}$sufficiently close to $K_{+}$(so that $g_{0}^{\prime}$ is small), and so (6) is positive.

We can then compute the Liouville vector field associated to $h_{\epsilon} \alpha$, which is given by

$$
V_{\epsilon}:=\frac{h_{\epsilon}}{h_{\epsilon}+h_{\epsilon}^{\prime} d r(V)} V .
$$

(Recall that $V$ is the Liouville vector field associated to $\alpha$.) Therefore it is a positive multiple of $V$, for suitably small $h_{\epsilon}^{\prime}$, for a multiple which is as arbitrarily close to 1 as desired, and coincides with $\pm \partial_{r}$ over $\{|r| \geq 2\}$. It follows that the Liouville vector field associated to $\Lambda_{\epsilon}$ is $Z_{\epsilon}:=X+V_{\epsilon}$. Here, $X$ is the Liouville vector field of $\lambda$, defined by

$$
X= \begin{cases}X_{+}, & \text {on }\{r>1-\delta\} \\ X_{-}, & \text {on }\{r<-1+\delta\} \\ \partial_{t}, & \text { on }\{|r|<1\},\end{cases}
$$

where $X_{ \pm}$is the Liouville vector field associated to $d \lambda_{ \pm}$. Since $V_{\epsilon}$ can be made as arbitrarily close to $V$ as we wish, $Z_{\epsilon}$ is transverse to $M^{L, Q}$ for every $L, Q$, and therefore, for the fixed choice $L=1, Q=0$, the manifold $\left(M=M^{1,0},\left.\Lambda_{\epsilon}\right|_{M}\right)$ is contact. This proves the first part of Lemma 3.2, through the observation that $\left.\Lambda_{\epsilon}\right|_{M}$ (for $\Lambda_{\epsilon}$ as defined in (5)) coincides with (3) after setting $f_{\epsilon}(r)=\epsilon e^{G_{ \pm}(r)}$.

For the second part, I point out that model A in [18] was also constructed via the double completion, but with the Liouville form $\Lambda_{\epsilon}$ replaced by the Liouville form $\lambda_{\sigma}=\sigma \alpha+\lambda$, where $\sigma=\mathbb{R} \rightarrow \mathbb{R}^{+}$is a suitable smooth function (see [18, Section 3.1, pp. 37-38]). Then linearly homotope between the Liouville forms $\lambda_{\sigma}$ and $\Lambda_{\epsilon}$ via

$$
\Lambda_{t}=g_{t}\left(\sigma, h_{\epsilon}\right) \alpha+g_{t}(1, \epsilon) \lambda,
$$

where $g_{t}(A, B)=(1-t) A+t B$ is the linear interpolation between the quantities $A$ and $B$. Again by Thurston's trick, to show that $\Lambda_{t}$ is Liouville for all $t$ we only need to check that $g_{t}\left(\sigma, h_{\epsilon}\right) \alpha$ and $g_{t}(1, \epsilon) \lambda$ are Liouville forms in the horizontal and vertical directions, respectively, and that the Liouville vector field $Z_{t}$ associated to $\Lambda_{t}$ is transverse to $M$ 
for every $t$. The 1 -form $g_{t}(1, \epsilon) \lambda$ is obviously Liouville, and its Liouville vector field is clearly a multiple of that of $\lambda$. For the 1 -form $g_{t}\left(\sigma, h_{\epsilon}\right) \alpha$, the computation is the same as for $h_{\epsilon} \alpha$, with the expression of the corresponding Liouville vector field being the same as for $V_{\epsilon}$ but with $h_{\epsilon}$ replaced by $g_{t}\left(\sigma, h_{\epsilon}\right)$. The path $\left.\Lambda_{t}\right|_{M}$ is then an isotopy of contact forms interpolating between model A and model B. This proves Lemma 3.2.

\subsection{Deformation to an SHS along the cylindrical region}

To obtain an SHS deforming the contact data, which I will need to construct holomorphic hypersurfaces, I homotope the Liouville vector field over $M_{Y}$ (the model B paper) as follows. Choose a bump function $\kappa: \mathbb{R} \rightarrow \mathbb{R}$ which equals 1 outside of the unit interval and 0 in $[-1+\delta, 1-\delta]$, with $\operatorname{sign}\left(\kappa^{\prime}(r)\right)=\operatorname{sign}(r) \neq 0$ for $r \in\{1-\delta<|r|<1\}$.

Define

$$
W_{\epsilon}^{s}:= \begin{cases}Z_{\epsilon}, & \text { in } E^{\infty, \infty} \backslash\left(\{|r| \leq 1\} \cap E^{\infty, \infty}(t)\right) \\ X+(s \kappa(r)+1-s) V_{\epsilon}, & \text { in }\{|r| \leq 1\} \cap E^{\infty, \infty}(t) .\end{cases}
$$

We can check that

$$
\mathcal{H}_{\epsilon}^{s}:=\left(\Lambda_{\epsilon}^{s}, \Omega_{\epsilon}\right)=\left(\left.i_{W_{s}^{\epsilon}} d \Lambda_{\epsilon}\right|_{T M},\left.d \Lambda_{\epsilon}\right|_{T M}\right)_{s \in[0,1]}
$$

is a family of SHSs on $M$ which deform model B (obtained for $s=0$ ) and such that $\Lambda_{\epsilon}^{s}$ is contact for $s<1$. This follows from the fact that the Reeb dynamics are invariant when $M$ is flowed via $W_{\epsilon}^{s}$ inside $E^{\infty, \infty}$ (see (8)) - that is, $W_{\epsilon}^{s}$ is stabilising - or via a straightforward check which I leave to the reader.

Focusing now on the case where $L=1, Q=0$, we have $\Lambda_{\epsilon}^{s}=\epsilon \lambda+(s \kappa+1-s) h_{\epsilon} \alpha$, which we can explicitly write as

$$
\Lambda_{\epsilon}^{s}= \begin{cases}\epsilon \lambda_{ \pm}+h_{\epsilon} \alpha_{ \pm}, & \text {in } M_{P}^{ \pm}=Y \times(\Sigma \backslash \mathcal{U}) \\ f_{\epsilon}(r) d \theta+\left(s \kappa\left(F_{ \pm}(r) \pm 1\right)+1-s\right) h_{\epsilon} \alpha, & \text { in } M_{C}^{ \pm}=Y \times\left(\mathbb{R}^{ \pm} \cap\{|r|>1-\delta\}\right) \times S^{1} \\ \epsilon d \theta+(1-s) h_{\epsilon} \alpha, & \text { in } M_{Y}=Y \times[-1+\delta, 1-\delta] \times S^{1},\end{cases}
$$

where again we denote $f_{\epsilon}(r)=\epsilon e^{G_{ \pm}(r)}($ cf. (3)) .

One can check that the Reeb vector field $R_{\epsilon}^{s}$ associated to $\mathcal{H}_{\epsilon}^{s}$ is given by

$$
R_{\epsilon}^{s}= \begin{cases}R_{\epsilon}, & \text { in } M_{P}^{ \pm} \\ \frac{1}{D_{\epsilon}^{s}+f_{\epsilon} h_{\epsilon} \frac{\partial \alpha_{r}}{\partial r}\left(R_{r}\right)}\left(\left(h_{\epsilon}^{\prime}+h_{\epsilon} \frac{\partial \alpha_{r}}{\partial r}\left(R_{r}\right)\right) \partial_{\theta}-f_{\epsilon}^{\prime} R_{r}\right), & \text { in } M_{C}^{ \pm} \\ \partial_{\theta} / \epsilon, & \text { in } M_{Y},\end{cases}
$$

where $D_{\epsilon}^{s}:=f_{\epsilon} h_{\epsilon}^{\prime}-\left(s \kappa\left(F_{ \pm} \pm 1\right)+1-s\right) h_{\epsilon} f_{\epsilon}^{\prime}$.

Every pair $(p, \gamma)$ consisting of a critical point $p$ of $h_{ \pm}$and a closed $R_{ \pm}$-orbit $\gamma$ gives rise to a closed Reeb orbit of $R_{\epsilon}^{s}$ of the form $\gamma_{p}:=(\gamma, p) \in Y \times \Sigma$. The closed Reeb orbits which do not correspond to critical points of $h_{ \pm}$can be made to have arbitrarily large period by taking $\epsilon$ sufficiently close to zero. Therefore we can find an action threshold $T_{\epsilon}>0$ ( $s$-independent) such that $\lim _{\epsilon \rightarrow 0} T_{\epsilon}=+\infty$ and such that every closed Reeb orbit of $R_{\epsilon}^{s}$ of action less than $T_{\epsilon}$ either lies in $Y \times \mathcal{U}$ or is a cover $\gamma_{p}^{l}$ of a simple closed Reeb orbit $\gamma_{p}$, for $l \leq N_{\epsilon}$, where $N_{\epsilon}:=\max \left\{n \in \mathbb{N}: \gamma^{n}\right.$ has action less than $\left.T_{\epsilon}\right\}$. 
The following lemma will be used in the proof of Theorem 1.5:

Lemma 3.4. The 2-form $\omega=d\left(e^{\epsilon} \Lambda_{\epsilon}^{s}\right)$ is symplectic in $\left(0, \epsilon_{0}\right] \times M$ for sufficiently small $\epsilon_{0}>0$ and for $0 \leq s<1$, and induces the orientation given by the natural product orientation (where the orientation on $M$ is as described before Lemma 3.2).

Proof: It follows by direct computations [16].

\subsection{Compatible almost complex structure}

I proceed now to define an $\mathcal{H}_{\epsilon}^{s}$-compatible (and nongeneric) almost complex structure $J_{\epsilon}^{s}$ in $\mathbb{R} \times M$. I define it on $\xi_{\epsilon}^{s}=\operatorname{ker} \Lambda_{\epsilon}^{s}$ and extend it in a cylindrical way.

Write

$$
\xi_{\epsilon}^{s}= \begin{cases}\xi_{ \pm} \oplus L_{\epsilon}^{s}, & \text { in } M_{P}^{ \pm} \cup M_{C}^{ \pm} \\ T(Y \times I), & \text { in } M_{Y}\end{cases}
$$

where $T \widetilde{(Y \times I)}=\left\{v-(1-s) h_{\epsilon} \frac{\alpha(v)}{\epsilon} \partial_{\theta}: v \in T(Y \times I)\right\}$ and $L_{\epsilon}^{s}$ is a bundle of (real) rank 2. The latter may be written as

$$
L_{\epsilon}^{s}= \begin{cases}T \Sigma_{ \pm}, & \text {in } \operatorname{crit}\left(h_{ \pm}\right) \subseteq M_{P}^{ \pm} \\ \left\langle\nabla h_{ \pm}, h_{\epsilon} X_{h_{ \pm}}-\epsilon\left|\nabla h_{ \pm}\right|^{2} R_{ \pm}\right\rangle, & \text {in } M_{P}^{ \pm} \backslash \operatorname{crit}\left(h_{ \pm}\right) \\ \left\langle V= \pm \partial_{r}, W\right\rangle, & \text { in } M_{C}^{ \pm},\end{cases}
$$

where

$$
W:=\mp f_{\epsilon} R_{r} \pm\left(s \kappa\left(F_{ \pm} \pm 1\right)+1-s\right) h_{\epsilon} \partial_{\theta} .
$$

Over the region $M_{P}^{ \pm}=Y \times\left(\Sigma_{ \pm} \backslash \mathcal{U}\right)$, the differential of the projection $\pi: Y \times\left(\Sigma_{ \pm} \backslash \mathcal{U}\right) \rightarrow$ $\Sigma_{ \pm} \backslash \mathcal{U}$ is an isomorphism for every $\epsilon$ when restricted to $L_{\epsilon}^{s}$. We can then define

$$
\left.J_{\epsilon}^{s}\right|_{\xi_{\epsilon}^{s}}=J_{ \pm} \oplus\left(\left.\pi\right|_{L_{\epsilon}^{s}}\right)^{*} j_{ \pm}
$$

with respect to the splitting above in equation 9 , where $J_{ \pm}$is a $d \alpha_{ \pm}$-compatible almost complex structure in $\xi_{ \pm}$and $j_{ \pm}$is a $d \lambda_{ \pm}$-compatible almost complex structure on $\Sigma_{ \pm}$ which satisfies $j_{ \pm}\left(\partial_{t_{ \pm}}\right)=\partial_{\theta}$ on the collar neighbourhoods.

Choose now a $d \alpha$-compatible almost complex structure $J_{0}$ in the Liouville domain $Y \times I$ which is cylindrical in the cylindrical ends $\{|r|>1-\delta\}$. Along these, its restriction to $\xi_{r}$ coincides with $J_{ \pm}$.

Over the region $Y \times \mathcal{U}=Y \times I \times S^{1}$, the projection $\pi_{Y}: Y \times \mathcal{U} \rightarrow Y \times I$ gives an isomorphism

$$
\left.d \pi_{Y}\right|_{\xi_{\epsilon}^{s}}: \xi_{\epsilon}^{s}=T \widetilde{(Y \times I)} \stackrel{\simeq}{\longrightarrow} T(Y \times I),
$$

and thus $J_{\epsilon}^{S}=\pi_{Y}^{*} J_{0}$ is an almost complex structure on $\xi_{\epsilon}^{s}$.

In order to glue the two definitions along the region $\mathbb{R} \times M_{C}^{ \pm}$, compute that $J_{\epsilon}^{s}(W)=$ $\left.s \kappa\left(F_{ \pm} \pm 1\right)+1-s\right) h_{\epsilon} \partial_{r}$ close to $r= \pm 1$ and $J_{\epsilon}^{s}(W)=f_{\epsilon} \partial_{r}$ close to $r= \pm 1 \mp \delta$. We can then define $J_{\epsilon}^{s}(W)=\beta_{\epsilon, \pm}^{s}(r) \partial_{r}$ for suitable interpolating functions $\beta_{\epsilon, \pm}^{s}(r)$.

We thus get a well-defined almost complex structure $J_{\epsilon}^{s}$ over the whole model. 


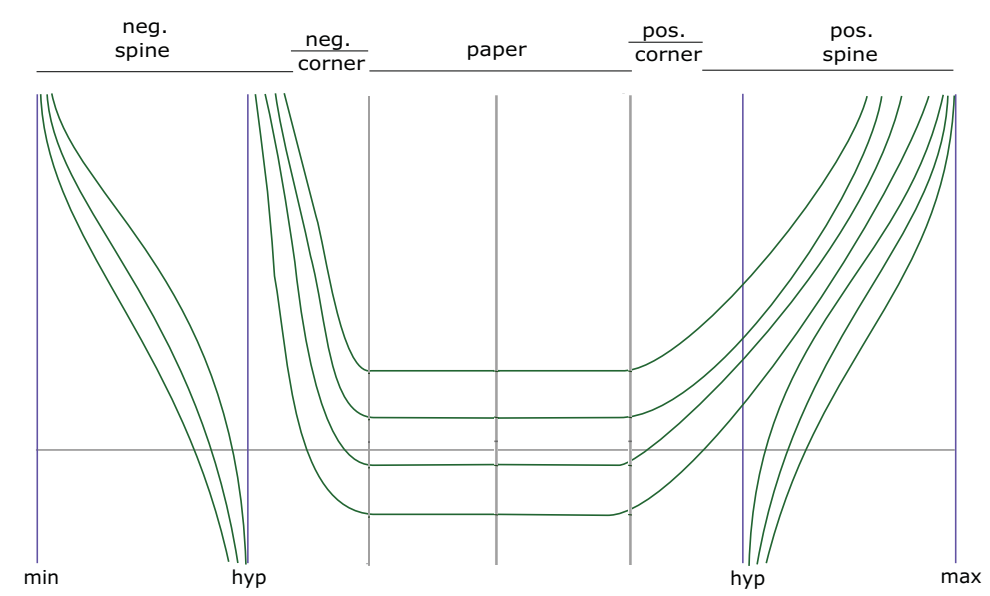

Figure 4. Foliation by holomorphic hypersurfaces. The vertical lines are the cylindrical hypersurfaces corresponding to critical points $\min$ (minimum), hyp (hyperbolic) an $\max$ (maximum), and the nonvertical ones are the noncylindrical $H_{a}^{v}$.

Compatibility. One can check that $J_{\epsilon}^{s}$ is $\mathcal{H}_{\epsilon}^{s}$-compatible by explicit computation on these splittings (cf. [16, pp. 47-48] for the model A case).

Remark 3.5. Observe that (9) is holomorphic. One can directly check that $c_{1}\left(\xi_{\epsilon}^{1}\right)=0$ whenever $c_{1}\left(\xi_{ \pm}\right)=0$.

\subsection{Foliation by holomorphic hypersurfaces}

The goal of this section is to construct a foliation of $\mathbb{R} \times M$ by holomorphic hypersurfaces (where the almost complex structure is the one compatible with the noncontact SHS $\mathcal{H}_{\epsilon}^{1}$ ). Recall the Morse functions $h_{ \pm}$, whose gradient flow we assume to be Morse-Smale.

Proposition 3.6. For $\epsilon \in\left(0, \epsilon_{0}\right]$, there exists a foliation $\mathcal{F}=\bigcup_{a, v} H_{a}^{\nu}$ of the symplectisation of $\mathcal{H}_{\epsilon}^{1}$ by $J_{\epsilon}^{1}$-holomorphic hypersurfaces, which come in three types (see Figure 4 ):

1. (Cylindrical hypersurfaces over critical points) For $p \in \operatorname{crit}\left(h_{ \pm}\right)$, there exists a cylindrical hypersurface of the form $\mathbb{R} \times H_{p}$, where $H_{p}:=Y \times\{p\}$, a copy of the symplectisation of $\left(Y, \alpha_{ \pm}\right)$.

2. (Positive/negative flow-line hypersurfaces contained in one side of the dividing set) These are parametrised by

$$
H_{a}^{v}=\{(a(s), v(s)): s \in \mathbb{R}\} \times Y,
$$

where $a: \mathbb{R} \rightarrow \mathbb{R}$ is a proper function and $v: \mathbb{R} \rightarrow \operatorname{int}\left(\Sigma_{ \pm}\right)$is a nonconstant negative/positive reparametrisation of a flow line for $h_{ \pm}$.

If $\lim _{s \rightarrow \pm \infty} v(s)=p_{ \pm}$, then $H_{a}^{v}$ is asymptotically cylindrical over $H_{p_{ \pm}}$(see $[16,11]$ for a definition) and has exactly one positive and one negative end. If $v$ is contained in $\Sigma_{+}$(and thus $v$ connects the maximum to a hyperbolic point), the positive end 
corresponds to the maximum. If $v$ is contained in $\Sigma_{-}$(and thus $v$ connects the minimum to a hyperbolic point), it corresponds to the minimum.

3. (Page-like hypersurfaces crossing sides of the dividing set) These may be written as

$$
H_{a}^{v}=H_{a}^{\nu,-} \bigcup H_{a}^{v, c s t} \bigcup H_{a}^{v,+}
$$

where

$$
\begin{gathered}
H_{a}^{v, c s t}=\{a\} \times Y \times[-1,1] \subseteq \mathbb{R} \times M_{Y} \\
H_{a}^{\nu,+}:=\left\{\left(a_{+}(s), v_{+}(s)\right): s \in[0,+\infty)\right\} \times Y \subseteq \mathbb{R} \times M_{P}^{+} \\
H_{a}^{\nu,-}:=\left\{\left(a_{-}(s), \nu_{-}(s)\right): s \in(-\infty, 0]\right\} \times Y \subseteq \mathbb{R} \times M_{P}^{-} .
\end{gathered}
$$

Here, $a_{+}:[0,+\infty) \rightarrow \mathbb{R}$ and $a_{-}:(-\infty, 0] \rightarrow \mathbb{R}$ are proper functions, and $v=v_{+} \sqcup v_{-}$ for $\nu_{+}:[0,+\infty) \rightarrow \Sigma_{+}$a nonconstant reparametrisation of a negative Morse flow line of $h_{+}$and $\nu_{-}:(-\infty, 0] \rightarrow \Sigma_{-}$a nonconstant reparametrisation of a positive Morse flow line of $h_{-}$, both satisfying $v_{ \pm}(0) \in \partial \Sigma_{ \pm}$. If $\lim _{s \rightarrow \pm \infty} v_{ \pm}(s)=p_{ \pm}$, the hypersurface $H_{a}^{v}$ is asymptotically cylindrical over $H_{p_{ \pm}}$, having exactly two positive ends.

Proof: Along the paper, compute that

$$
J_{\epsilon}^{1} R_{ \pm}=F \nabla^{g_{ \pm}} h_{ \pm}+G \partial_{a}
$$

where

$$
F=\frac{\epsilon h_{\epsilon}}{h_{\epsilon}+\epsilon^{2}\left|\nabla h_{ \pm}\right|^{2}}, G=-h_{\epsilon} .
$$

Observe that $F>0, G<0$. Then

$$
\left\langle R_{ \pm}, J_{\epsilon}^{1} R_{ \pm}\right\rangle \oplus \xi_{ \pm}=\left\langle F \nabla^{g_{ \pm}} h_{ \pm}+G \partial_{a}\right\rangle \oplus T Y
$$

a $J_{\epsilon}^{1}$-invariant and integrable distribution over $M_{P}^{ \pm}$. Integrating this distribution, we get the hypersurfaces $H_{a}^{v}$ as in the proposition. For instance, for the hypersurfaces of the second type, take $a$ and $v$ to satisfy

$$
\begin{gathered}
\dot{v}(s)=\mp F(v(s)) \nabla^{g_{ \pm}} h_{ \pm}(v(s)) \\
\dot{a}(s)=\mp G(v(s)) .
\end{gathered}
$$

For hypersurfaces of the third type, one can check that for $a_{ \pm}$and $v_{ \pm}$satisfying the same ODEs, we can glue each piece as in the statement to get a hypersurface $H_{a}^{v}$ whose tangent space is

$$
T H_{a}^{v}= \begin{cases}\left\langle R_{ \pm}, J_{\epsilon}^{1} R_{ \pm}\right\rangle \oplus \xi_{ \pm}, & \text {over } M_{P}^{ \pm} \cup M_{C}^{ \pm} \\ T(Y \times I), & \text { over } M_{Y},\end{cases}
$$

which is clearly $J_{\epsilon}^{1}$-invariant. The assertion about the asymptotic behaviour of the ends of 
$H_{a}^{v}$ is deduced by the fact that orientations change as we move from one side to the other. The condition that the holomorphic hypersurfaces $H_{a}^{v}$ are asymptotically cylindrical follows from the fact that they project to $\Sigma$ as the Morse flow line $v$ [16].

Remark 3.7. For a flow-line hypersurface $H_{a}^{v}$ which does not cross the dividing set, and $p_{ \pm}=\lim _{s \rightarrow \pm \infty} v(s)$ a critical point, the projection map

$$
\begin{aligned}
P_{a}^{v}: H_{a}^{v}= & \{(a(s), v(s)): s \in \mathbb{R}\} \times Y \rightarrow \mathbb{R} \times H_{p_{ \pm}}, \\
& (a(s), v(s), y) \mapsto\left(s, y, p_{ \pm}\right),
\end{aligned}
$$

gives an identification of $H_{a}^{v}$ with $\mathbb{R} \times H_{p_{ \pm}}$, which, up to replacing $s$ by $-s$ over $M_{P}^{-}$, is a biholomorphism. In particular, it maps holomorphic curves in $H_{a}^{v}$ to holomorphic curves in $\mathbb{R} \times H_{p_{ \pm}}$. The hypersurfaces which do cross sides are cylindrical completions of the Liouville domain $Y \times I$, and their cylindrical ends are identified with the symplectisation of $\left(Y, \alpha_{ \pm}\right)$via the a map as above.

Remark 3.8. Assume that ( $Y, \alpha_{ \pm}$) both satisfy the Weinstein conjecture, so that there are closed $\alpha_{ \pm}$-Reeb orbits. Then the hypersurfaces $H_{a}^{v}$ which stay on the same side of the dividing set contain finite-energy $J_{\epsilon}^{s}$-holomorphic curves (for every $s$ ). These are of the form

$$
\begin{gathered}
u_{\gamma}^{a}: \mathbb{R} \times S^{1} \rightarrow \mathbb{R} \times M \\
u_{\gamma}^{a}(s, t)=(a(s), v(s), \eta(t)),
\end{gathered}
$$

where $v$ and $a$ are as before and $\eta$ is a closed $\alpha_{ \pm}$-Reeb orbit satisfying $\lim _{s \rightarrow \pm \infty} v(s)=p_{ \pm}$ for $p_{ \pm}$critical point. The asymptotic behaviour of $u_{\gamma}^{a}$ is thus the same as the hypersurface $H_{a}^{v}$ containing it (namely, the positive/negative cylindrical ends of the curve lie in the positive/negative cylindrical ends of the hypersurface, so that the Reeb asymptotics of the curve are contained in the cylindrical asymptotics of the hypersurface). These cylinders correspond to trivial cylinders under the identification of Remark 3.7.

For a general choice of closed manifold $Y$ (satisfying the standing assumption that $Y \times I$ is Liouville), there is no reason why holomorphic cylinders crossing sides should exist, and in fact there are examples for which they do not (e.g., for $Y$ the unit cotangent bundle of a closed hyperbolic surface; see Section 4.2, fact C).

Remark 3.9. In the language and notation of $[16,11]$, the holomorphic foliation $\mathcal{F}$ is compatible with the $H_{\epsilon}$-admissible fibration $\left(\Sigma, \pi_{\Sigma}, Y, H_{\epsilon}\right)$. Here, $\pi_{\Sigma}: M=Y \times \Sigma \rightarrow \Sigma$ is the natural projection, and $H_{\epsilon}$ is a suitably defined Morse function on $\Sigma$ having the same critical points as $h_{ \pm}$, but up to orientation reversal on $\Sigma_{+}$, so that its unique maximum is the minimum of $h_{+}$[16]. The binding of $\mathcal{F}$ is $\bigcup_{p \in \operatorname{crit}\left(H_{\epsilon}\right)} H_{p}$, where each $H_{p}$ is a strong stable hypersurface. The sign function $\operatorname{sign}: \operatorname{crit}\left(H_{\epsilon}\right) \rightarrow\{-1,1\}$ is defined by $\operatorname{sign}(p)= \pm 1$ if $p \in \operatorname{crit}\left(h_{ \pm}\right) \subseteq \Sigma_{ \pm}$.

I will use this fact in Section 3.6, together with the results from $[16,11]$, to show that every holomorphic curve in $\mathbb{R} \times M$ with positive asymptotics corresponding to critical points has to lie in a leaf of $\mathcal{F}$. 


\subsection{Index computations}

For closed Reeb orbits $\gamma_{p}: S^{1} \rightarrow M$ of the form $\gamma_{p}(t)=(\gamma(t), p)$, for $p \in \operatorname{crit}\left(h_{ \pm}\right)$and $\gamma$ a closed $\alpha_{ \pm}$-Reeb orbit, we have a natural identification

$$
\Gamma\left(\gamma_{p}^{*} \xi_{\epsilon}^{s}\right)=\Gamma\left(\gamma^{*} \xi_{ \pm}\right) \oplus \Gamma\left(\left.L_{\epsilon}^{s}\right|_{p}\right)=\Gamma\left(\gamma^{*} \xi_{ \pm}\right) \oplus \Gamma\left(T_{p} \Sigma_{ \pm}\right)
$$

where the second summand denotes the space of sections of the trivial line bundle over $S^{1}$ with fibre $\left.L_{\epsilon}^{s}\right|_{p}=T_{p} \Sigma_{ \pm}$. This splitting corresponds to splitting into tangent and normal components with respect to the hypersurface $H_{p}$ containing $\gamma_{p}$. Any trivialisation $\tau$ of $\gamma^{*} \xi_{ \pm}$extends naturally to a trivialisation of $\gamma_{p}^{*} \xi_{\epsilon}^{s}$, which I shall also denote by $\tau$.

Proposition 3.10. Consider a trivialisation $\tau$ of $\gamma^{*} \xi_{ \pm}$over the simply covered $\alpha_{ \pm}$Reeb orbit $\gamma$, inducing a natural trivialisation $\tau^{l}$ of $\left(\gamma^{l}\right)^{*} \xi_{ \pm}$for every $l$. Then for each sufficiently small $\epsilon>0$ there exists a covering threshold $N_{\epsilon}$, satisfying $\lim _{\epsilon \rightarrow 0} N_{\epsilon}=+\infty$, such that the Conley-Zehnder index of $\gamma_{p}^{l}$ for $l \leq N_{\epsilon}$ with respect to the induced trivialisation of $\left(\gamma_{p}^{l}\right)^{*} \xi_{\epsilon}^{s}$ given by (12) is

$$
\mu_{C Z}^{\tau^{l}}\left(\gamma_{p}^{l}\right)= \begin{cases}\mu_{C Z}^{\tau^{l}}\left(\gamma^{l}\right)+1, & \text { if } p \text { is the maximum or minimum } \\ \mu_{C Z}^{\tau^{l}}\left(\gamma^{l}\right), & \text { if } p \text { is hyperbolic. }\end{cases}
$$

Here, $\mu_{C Z}^{\tau}(\gamma)$ denotes the Conley-Zehnder index of $\gamma$ in the case where this Reeb orbit is nondegenerate or Morse-Bott.

Remark 3.11. In the Morse-Bott case, we need to add suitable weights adapted to the spectral gap of the corresponding operator to obtain $\mu_{C Z}^{\tau}(\gamma)$, as explained in, for example, [11].

Proof: I sketch the proof (see [16, Prop. 4.9] for full details). Consider the asymptotic operator associated to the Reeb orbit $\gamma_{p}^{l}$. This operator looks like

$$
\begin{gathered}
\mathbf{A}_{\gamma_{p}^{l}}: W^{1,2}\left(\left(\gamma_{p}^{l}\right)^{*} \xi_{\epsilon}^{s}\right) \rightarrow L^{2}\left(\left(\gamma_{p}^{l}\right)^{*} \xi_{\epsilon}^{s}\right) \\
\mathbf{A}_{\gamma_{p}^{l}} \eta=-J_{\epsilon}^{s}\left(\nabla_{t} \eta-T \nabla_{\eta} R_{\epsilon}\right),
\end{gathered}
$$

where $\nabla$ is any symmetric connection in $M$ and

$$
T=\Lambda_{\epsilon}\left(\dot{\gamma}_{p}^{l}\right)=l h_{\epsilon}(p) \alpha_{ \pm}(\dot{\gamma})=l h_{\epsilon}(p) T_{\gamma}
$$

is the action of $\gamma_{p}^{l}\left(T_{\gamma}=\alpha_{ \pm}(\dot{\gamma})\right.$ is the action of $\left.\gamma\right)$. The splitting of this operator into tangent and normal components with respect to the hypersurface $H_{p}$ is a direct sum of the form

$$
\mathbf{A}_{\gamma_{p}^{l}}=\mathbf{A}_{\gamma^{l}} \oplus \mathbf{A}_{\epsilon, \gamma^{l}}^{ \pm}
$$

where

$$
\mathbf{A}_{\epsilon, \gamma^{l}}^{ \pm}=-i \nabla_{t}-\epsilon l T_{\gamma} \nabla_{p}^{2} h_{ \pm} .
$$

The operator $\mathbf{A}_{\epsilon, \gamma^{l}}^{ \pm}$is the normal asymptotic operator associated to $\gamma^{l}$ acting on $\Gamma\left(T_{p} \Sigma\right)$. 
The additivity of $\mu_{C Z}$ implies that

$$
\mu_{C Z}^{\tau^{l}}\left(\gamma_{p}^{l}\right)=\mu_{C Z}^{\tau^{l}}\left(\gamma^{l}\right)+\mu_{C Z}\left(\mathbf{A}_{\epsilon, \gamma^{l}}^{ \pm}\right)
$$

For $\epsilon>0$ small enough, the operator $\mathbf{A}_{\epsilon, \gamma^{l}}^{ \pm}$is nondegenerate, and the smaller $\epsilon$ is, the larger we are allowed to take $T_{\gamma^{l}}=l T_{\gamma}$. Since every orbit of action less than $T_{\epsilon}$ satisfies the condition that this operator is nondegenerate, we have

$$
\mu_{C Z}\left(\mathbf{A}_{\epsilon, \gamma^{l}}^{ \pm}\right)=1-i n d_{p}\left(h_{ \pm}\right) \in\{0,1\}
$$

and this computation is valid for $l \leq N_{\epsilon}=\max \left\{n \in \mathbb{N}: \gamma^{l}\right.$ has action $\left.<T_{\epsilon}\right\}$.

\subsection{Holomorphic curves lie in hypersurfaces}

In this section, I shall make use of intersection theory for punctured holomorphic curves and holomorphic hypersurfaces, as outlined in [16, 11], to which I refer for the relevant definitions and notation.

If we assume by perturbing that $\left(Y, \alpha_{ \pm}\right)$are nondegenerate contact manifolds, then we are in the situation of $[16,11]$, as can be easily checked. For instance, I have already observed that the splitting of the asymptotic operator $\mathbf{A}_{\gamma_{p}^{l}}$ into tangent and normal components is given by $\mathbf{A}_{\gamma_{p}^{l}}=\mathbf{A}_{\gamma^{l}} \oplus \mathbf{A}_{\epsilon, \gamma_{p}^{l}}^{ \pm}$, where the normal operator has Conley-Zehnder index $\mu_{C Z}\left(\mathbf{A}_{\epsilon, \gamma_{p}^{l}}^{ \pm}\right)=1-\operatorname{ind}\left(h_{ \pm}\right)=\operatorname{sign}(p)\left(\operatorname{ind}_{p}\left(H_{\epsilon}\right)-1\right)$, where $H_{\epsilon}$ and the sign function are the ones of Remark 3.9.

Proposition 3.12. Suppose $u: \dot{S} \rightarrow \mathbb{R} \times M$ is a finite-energy $J_{\epsilon}^{1}$-holomorphic curve which has all of its positive ends asymptotic to Reeb orbits of the form $\gamma_{p}^{l}$, with $l \leq N_{\epsilon}$. Then the image of $u$ is contained in a leaf of the foliation $\mathcal{F}$.

Proof: Given such a curve $u$, by Stokes' theorem we have that its negative ends have action bounded by $T_{\epsilon}$, and so also correspond to critical points. Since all of its asymptotics project to $\Sigma$ as points, we can define a map $v:=\pi_{\Sigma} \circ u: S \rightarrow \Sigma$ between closed surfaces. We are therefore in the situation of [11], which, since the map $\operatorname{sign}: \operatorname{crit}\left(H_{\epsilon}\right) \rightarrow\{-1,1\}$ is surjective, implies the result.

Remark 3.13. Proposition 3.12 reduces the study of $J_{\epsilon}^{1}$-holomorphic curves/buildings inside $\mathbb{R} \times M$ to the study of $J_{0}$-curves/buildings inside the completion $W_{0}$ of the cylindrical Liouville semifilling $Y \times I$. Recall that $J_{0}$ is any cylindrical $d \alpha$-compatible almost complex structure in $Y \times I$, and $W_{0}$ is obtained by attaching cylindrical ends to $\partial(Y \times I)=\left(Y_{-}, \alpha_{-}\right) \bigsqcup\left(Y_{+}, \alpha_{+}\right)$. Holomorphic buildings inside $W_{0}$ are distributed along a main level, which can be identified with $W_{0}$ itself, and perhaps several upper levels, which come in two types depending on whether they correspond to the symplectisation of $\left(Y, \alpha_{+}\right)$or $\left(Y, \alpha_{-}\right)$(see Figure 5$)$. In further sections, I will refer to these upper levels as right or left, respectively. 


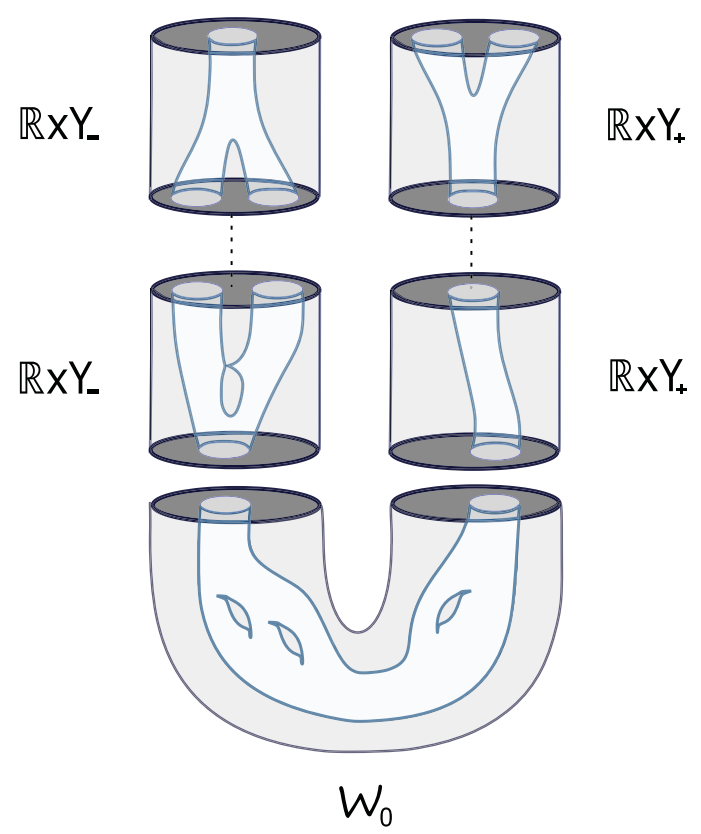

Figure 5. A building in $W_{0}=\widehat{Y \times I}$, the completion of the cylindrical Liouville semifilling $Y \times I$.

\subsection{Regularity inside a hypersurface versus regularity in the symplectisation for curves of genus zero}

Consider $u: \dot{S} \rightarrow \mathbb{R} \times M$, a finite-energy $J_{\epsilon}^{1}$-holomorphic curve with asymptotics of the form $\gamma_{p}^{l}, l \leq N_{\epsilon}$, and genus $g$. By Proposition 3.12, $u(\dot{S}) \subseteq H$ for some $H \in \mathcal{F}$. If $H$ is positively/negatively asymptotically cylindrical over $H_{p_{ \pm}}$, then the positive/negative asymptotics of $u$ are of the form $\gamma_{p_{ \pm}}^{l}$ for some simply covered orbit $\gamma \subseteq H_{ \pm}$. Denote by $\Gamma=\Gamma^{+} \cup \Gamma^{-}$the set of punctures of $u$ (where $\Gamma^{ \pm}$is the set of positive/negative punctures). Define a partition $\Gamma^{ \pm}=\Gamma_{0}^{ \pm} \cup \Gamma_{1}^{ \pm} \cup \Gamma_{2}^{ \pm}$, where $\Gamma_{i}^{ \pm}$denotes the set of positive/negative punctures for which the corresponding asymptotic is of the form $\gamma_{p}^{l}$, with $\operatorname{ind}_{p}\left(H_{\epsilon}\right)=i \in$ $\{0,1,2\}$.

Lemma 3.14. Assume that $u \subseteq H$ has genus $g=0$ and is Fredholm regular inside $H$, and that

$$
\# \Gamma_{0}^{-}+\# \Gamma_{1}^{-}+\# \Gamma_{1}^{+}+\# \Gamma_{2}^{-}<2 .
$$

Then $u$ is Fredholm regular in $\mathbb{R} \times M$.

Remark 3.15. Observe that since all the positive asymptotics of $u$ correspond to the same critical point of $H_{\epsilon}$ (and similarly for all the negative ones), $\# \Gamma_{i}^{ \pm} \neq 0$ implies $\# \Gamma_{j}^{ \pm}=0$ for $j \neq i$.

Proof: Consider the complex splitting $N_{u}=N_{u}^{H} \oplus u^{*} N_{H}$, where $N_{u}$ is the normal bundle to $u$ (in the generalised sense, in which $u$ is allowed to have critical points; see 
[11, Sec. 3.3]), $N_{u}^{H}$ is the normal bundle to $u$ inside $H$ and $N_{H}$ is the normal bundle to $H$ inside $\mathbb{R} \times M$ (a trivial complex line bundle). Then we get a matrix representation of the normal linearised Cauchy-Riemann operator $\mathbf{D}_{u}^{N}: W^{1,2, \delta_{0}}\left(N_{u}\right) \oplus$ $X_{\Gamma} \rightarrow L^{2, \delta_{0}}\left(\overline{H o m}_{\mathbb{C}}\left(\dot{S}, N_{u}\right)\right)$, given by

$$
\mathbf{D}_{u}^{N}=\left(\begin{array}{ll}
\mathbf{D}_{u ; T_{H}}^{N} & \mathbf{D}_{u ; N T}^{N} \\
\mathbf{D}_{u ; T N}^{N} & \mathbf{D}_{u ; N_{H}}^{N}
\end{array}\right),
$$

where

$$
\mathbf{D}_{u ; T_{H}}^{N}: W^{1,2, \delta_{0}}\left(N_{u}^{H}\right) \oplus X_{\Gamma} \rightarrow L^{2, \delta_{0}}\left(\overline{H o m}_{\mathbb{C}}\left(\dot{S}, N_{u}^{H}\right)\right)
$$

and

$$
\mathbf{D}_{u ; N_{H}}^{N}: W^{1,2, \delta_{0}}\left(u^{*} N_{H}\right) \rightarrow L^{2, \delta_{0}}\left(\overline{H o m}_{\mathbb{C}}\left(\dot{S}, u^{*} N_{H}\right)\right)
$$

are Cauchy-Riemann type operators and the off-diagonal operators are tensorial. Here, $X_{\Gamma}$ is a finite-dimensional vector space of smooth sections which are supported near infinity and parallel to the Morse-Bott submanifolds containing the asymptotics of $u$. Without loss of generality, we can assume that they are tangent to $H$.

The assumption that $u$ is regular inside $H$ is equivalent to the surjectivity of $\mathbf{D}_{u ; T H}^{N}$. Moreover, I claim that $\mathbf{D}_{u}^{N}$ can be assumed upper-triangular in this matrix representation, that is, that

$$
\mathbf{D}_{u ; T N}^{N}=0
$$

This, which is basically a generalisation of [11, Lemma 3.8], can be seen as follows: take any metric making the splitting $\left.T(\mathbb{R} \times M)\right|_{H}=T_{H} \oplus N_{H}$ orthogonal, and take $\nabla$ to be the associated Levi-Civita connection. This connection is symmetric and preserves this orthogonal splitting. If $\xi \in W^{1,2, \delta_{0}}\left(N_{u}^{H}\right)$, take a smooth path $t \rightarrow u_{t}$ of maps of class $W^{1, p, \delta}(\dot{S}, H)$, with image inside $H$, such that $\left.\partial_{t}\right|_{t=0} u_{t}=\xi, u_{0}=u$. Since $H$ is holomorphic, $\bar{\partial} u_{t} \in L^{2}\left(\overline{H o m}_{\mathbb{C}}\left(\dot{S}, u_{t}^{*} T H\right)\right)$ takes values in $u_{t}^{*} T H$. Then $\mathbf{D}_{u}^{N} \xi=\left.\pi_{N} \nabla_{t}\left(\bar{\partial} u_{t}\right)\right|_{t=0}$ takes values in $N_{u}^{H} \subseteq u^{*} T H$, since $\nabla$ preserves the splitting, and the claim follows.

Therefore, to show that $\mathbf{D}_{u}^{N}$ is surjective, it suffices to show that $\mathbf{D}_{u ; N_{H}}^{N}$ is. For this, we can use the automatic transversality criterion in [11], for the case of a line bundle. We need to check that

$$
\operatorname{ind}\left(\mathbf{D}_{u ; N_{H}}^{N}\right)>-2+2 g+\# \Gamma_{e v e n},
$$

where we denote by $\Gamma_{\text {even }}$ the set of punctures $z$ at which the Conley-Zehnder index of the asymptotic operator of $\mathbf{D}_{u ; N_{H}}^{N}$ at $z$ is even. Observe that $\Gamma_{\text {even }}=\Gamma_{1}^{+} \cup \Gamma_{1}^{-}$. Moreover, the operator $\mathbf{D}_{u ; N_{H}}^{N}$ is asymptotic at each puncture $z$ of $u$ to the normal asymptotic operator associated to the corresponding Reeb orbit $\gamma_{p_{z}}^{l_{z}}$ at $z$, whose Conley-Zehnder index is $\mu_{N}\left(\gamma_{p_{z}}^{l_{z}}\right)=\left|\operatorname{ind}_{p_{z}}\left(H_{\epsilon}\right)-1\right|$. By the Riemann-Roch formula, the Fredholm index 
of $\mathbf{D}_{u ; N_{H}}^{N}$ is

$$
\begin{aligned}
\operatorname{ind}\left(\mathbf{D}_{u ; N_{H}}^{N}\right) & =\chi(\dot{S})+\sum_{z \in \Gamma^{+}} \mu_{N}\left(\gamma_{p_{z}}^{l_{z}}\right)-\sum_{z \in \Gamma^{-}} \mu_{N}\left(\gamma_{p_{z}}^{l_{z}}\right) \\
& =2-2 g-\# \Gamma+\# \Gamma_{0}^{+}+\# \Gamma_{2}^{+}-\# \Gamma_{0}^{-}-\# \Gamma_{2}^{-} \\
& =2-2 g-2 \# \Gamma_{0}^{-}-2 \# \Gamma_{2}^{-}-\# \Gamma_{1}^{+}-\# \Gamma_{1}^{-},
\end{aligned}
$$

using $\# \Gamma=\sum_{i \in\{0,1,2\}} \# \Gamma_{i}^{ \pm}$and $c_{1}\left(N_{H}\right)=0$ in the natural trivialisation. Using $\# \Gamma_{\text {even }}=$ $\# \Gamma_{1}^{+}+\# \Gamma_{1}^{-},(17)$ can only be satisfied if $g=0$, in which case it is equivalent to (16).

Remark 3.16. Lemma 3.14 implies that the holomorphic cylinders of Remark 3.8 are regular index 1 cylinders in $\mathbb{R} \times M$. This generalises the situation in [11].

\subsection{Obstruction bundles}

In this section I will deal with the existence of obstruction bundles over buildings of holomorphic curves in $\mathbb{R} \times M$, to deal with the cases where Fredholm regularity fails. Given such a building $\mathbf{u}$, I want to compute the number of gluings one obtains from $\mathbf{u}$ for a generic perturbation of $J$ in the case where its components fail to be transversely cut out, but not too badly. For this, each component of $\mathbf{u}$ must consist of either a regular curve or a curve for which the dimension of the cokernel of the (normal) linearised CauchyRiemann operator is constant as the curve varies in its corresponding moduli space. I will refer to these curves as not too bad. If such is the case, one can construct an obstruction bundle $\mathcal{O}_{\mathbf{u}}$, an orientable bundle whose fibre is the direct sum of the cokernels of these operators, where the sum varies over the components of $\mathbf{u}$ which are not regular. The base of this bundle is the domain of a pregluing map, together with the parameter space keeping track of a fixed perturbation of $J$. Given such a generic (small) perturbation $t \mapsto J_{t}$ of $J=J_{0}$ through cyilindrical almost complex structures, the idea is to preglue the components of the $J_{0}$-holomorphic building $\mathbf{u}$ and impose that the resulting preglued curve be $J_{t}$-holomorphic. The resulting equation can be viewed as an obstruction to gluing in the form of a section of the obstruction bundle, whose algebraic count of zeroes is precisely the number of holomorphic gluings of $\mathbf{u}$ one obtains for sufficiently large gluing parameters. This technique, coming from algebraic geometry and Gromov-Witten theory, has been used, for example, in [10] and [11] in the context of ECH (see also [8] and [9] for gentler introductions).

I sketch the construction in a fairly general case, where curves are immersed and nonnodal. The nonimmersed case is slightly more technical, and for simplicity I will omit it. The normal bundle needs to be replaced with the restriction of the tangent bundle of $W$ to the curves in question, including a suitable Teichmüller slice parametrising complex structures on the domain, and divided by the action of the automorphism group. The nodal case just adds an extra $S^{1}$ factor as a gluing parameter for each node to the base of the obstruction bundle.

Setup. Let $t \mapsto J_{t}$ be a generic perturbation of $J=J_{0}, t \in[0,1]$, and consider a $J_{0}$-holomorphic building $\mathbf{u}=\left(u_{1}^{1}, \ldots, u_{r_{1}}^{1}, \ldots, u_{1}^{N}, \ldots, u_{r_{N}}^{N}\right)$ with $N \geq 1$ floors, $C \geq N$ 
components (where $C=\sum_{i=i}^{N} r_{i}$ ) denoted $u_{j}^{i}$, consisting of $\mathbb{R}$-translation classes of either regular curves or curves which are not too bad. I denote the not-too-bad curves by $u_{k}$, for $k \in\{1, \ldots, r\}$. Assume all of the $u_{j}^{i}$ are immersed. Assume also that $\mathbf{u}$ contains no trivial cylinder components, since they are regular and glue uniquely, so they do not affect the counts which I will consider. Then there is an orientable obstruction bundle

$$
\mathcal{O}_{\mathbf{u}} \rightarrow[0,1] \times \prod_{i=1}^{N-1}\left[R_{i},+\infty\right) \times \prod_{i=1}^{N}\left(\left(\prod_{j=1}^{r_{i}} \mathcal{M}_{j}^{i}\right) / \mathbb{R}\right):=\mathcal{B}_{\mathbf{u}}
$$

where $R_{i} \gg 0$ is a gluing parameter for gluing the $i$ th floor to the $(i+1)$ th floor of $\mathbf{u}$, and $\mathcal{M}_{j}^{i}$ is the moduli space of $J_{0}$-holomorphic curves containing $u_{j}^{i}$ (without modding out the $\mathbb{R}$-action on the target). The fibre of $\mathcal{O}_{\mathbf{u}}$ over an element in the base is $\bigoplus_{k=1}^{r}$ coker $\mathbf{D}_{k}^{N}$, where $\mathbf{D}_{k}^{N}$ is the normal linearised Cauchy-Riemann operator of the $k$ th not-too-bad curve $u_{k}$.

The base of the obstruction bundle is that of the pregluing map, and the interval $[0,1]$ is where the $t$ parameter varies. Observe that the case $r=0$ is when every component is regular, and we have a unique gluing. The case $N=1$ is also considered, in which there is no gluing to do but the components could be multiply covered, in which case I still wish to count their contributions in the form of a count of zeroes of a section of this bundle.

\section{Remark 3.17.}

i) (Orbibundle) In practice, one should check that the moduli spaces $\mathcal{M}_{j}^{i}$ corresponding to not-too-bad curves are orbifolds at worst. The obstruction bundle is then an orientable orbibundle, and the count of zeroes of a generic section is a weighted, rational count. The way to count is independent of the abstract perturbation scheme for polyfolds (see, e.g., [6] or [21] for a basic exposition on how to count zeroes of sections of orbibundles). In this article, the configurations that I care about will satisfy this orbifold condition, and the existence of obstruction bundles and a way of counting will be enough for my purposes.

ii) (Tangent space) Under the assumption of i), in practice one should also ask that

$$
T_{u_{k}} \mathcal{M}_{k}=\operatorname{ker} \mathbf{D}_{k}^{N}
$$

where $u_{k}$ is the $k$ th not-too-bad curve and $\mathcal{M}_{k}$ its corresponding moduli space. This condition is automatic for regular curves, but for not-too-bad curves a priori we only get the inclusion $T_{u_{k}} \mathcal{M}_{k} \subset \operatorname{ker} \mathbf{D}_{k}^{N}$. In the case of orbifolds, this is to be understood in the orbifold sense, where the tangent space is the quotient of an euclidean space by the action of the isotropy group. The condition is necessary to make sure that the counts of zeroes actually correspond to holomorphic gluings (cf. Remark 3.18).

iii) The fact that $\mathcal{O}_{\mathbf{u}}$ admits local trivialisations can be proven via standard results from nonlinear analysis (e.g., via the constant rank theorem for Fredholm operators [3, Corollary 3.1]). 
A section $\mathfrak{s}$ of this bundle, the obstruction section, is defined roughly as follows. Take pregluing data $(t, \mathbf{r}, \mathbf{v}) \in \mathcal{B}_{\mathbf{u}}$, where

$$
\begin{gathered}
\mathbf{r}=\left(r_{1}, \ldots, r_{N-1}\right), \\
\mathbf{v}=\left(v_{1}^{1}, \ldots, v_{r_{1}}^{1}, \ldots, v_{1}^{N}, \ldots, v_{r_{N}}^{N}\right) .
\end{gathered}
$$

Identify the $\mathbb{R}$-translation classes $v_{j}^{i}$ with representatives. Consider a tuple

$$
\Psi=\left(\psi_{1}^{1}, \ldots, \psi_{r_{1}}^{1}, \ldots, \psi_{1}^{N}, \ldots, \psi_{r_{N}}^{N}\right),
$$

where $\psi_{j}^{i}$ is a section of the normal bundle of $v_{j}^{i}$ belonging to a suitable Hilbert completion of the space of smooth sections.

We can construct a (only approximately $J_{t}$-holomorphic) preglued curve $\oplus_{(t, \mathbf{r}, \Psi)} \mathbf{v}$ out of the $J_{0}$-building $\mathbf{v}$ with the property that it converges to $\mathbf{v}$ as every component of $\mathbf{r}$ approaches $+\infty$. This is a standard construction, and is done by exponentiating $\psi_{j}^{i}$ along $v_{j}^{i}$ and using suitable smooth cutoff functions $\beta_{j}^{i}$, which equal 1 in the interior of $v_{j}^{i}$ and decay towards its cylindrical ends, translated in a way determined by the gluing parameters $\mathbf{r}$ (see, e.g., [11, Sec. 5.2]).

If we impose that $\oplus_{(t, \mathbf{r}, \Psi)} \mathbf{v}$ is $J_{t}$-holomorphic, we get an equation (cf. [11, Lemma 5.2]) of the form

$$
\sum_{i=1}^{N} \sum_{j=1}^{r_{i}} \beta_{j}^{i} \Theta_{j}^{i}(t, \mathbf{r}, \Psi)=0
$$

where

$$
\Theta_{j}^{i}(t, \mathbf{r}, \Psi)=\mathbf{D}_{j}^{i} \psi_{j}^{i}+\mathcal{F}_{j}^{i}(t, \mathbf{r}, \Psi) .
$$

Here, $\mathbf{D}_{j}^{i}$ is the linearised Cauchy-Riemann operator of $v_{j}^{i}$ for $t=0$, and the second summand involves extra terms arising from the patching construction, mostly nonlinear and depending only on the values of $\psi_{n}^{m}$ for which $v_{j}^{i}$ is adjacent to $v_{n}^{m}$.

For sufficiently small $t$ and fixed sufficiently large $R_{i}$, there exists a unique $\Psi=\Psi_{t, \mathbf{v}}$ such that

- $\psi_{j}^{i} \perp \operatorname{ker} \mathbf{D}_{j}^{i}$, for every $i, j$;

- $\Theta_{j}^{i}(t, \mathbf{r}, \Psi)=0$, if $u_{j}^{i}$ (or equivalently $v_{j}^{i}$ ) is regular;

- $\Theta_{j}^{i}(t, \mathbf{r}, \Psi) \perp \operatorname{im} \mathbf{D}_{j}^{i}$, if $u_{j}^{i}$ (or equivalently $v_{j}^{i}$ ) is not too bad

(cf. [8, Lemma 2.4, p. 8] and [11, Sec. 5.6, Prop. 5.6, Prop. 5.7]). Then we can define

$$
\mathfrak{s}(t, \mathbf{r}, \mathbf{v})=\bigoplus_{k=1}^{r} \pi_{k} \Theta_{k}\left(t, \mathbf{r}, \Psi_{t, \mathbf{v}}\right)=\bigoplus_{k=1}^{r} \pi_{k} \mathcal{F}_{k}\left(t, \mathbf{r}, \Psi_{\mathbf{t}, \mathbf{v}}\right),
$$

where $\pi_{k}$ is the orthogonal projection to coker $\mathbf{D}_{k}^{N}$. Therefore, there is a one-to-one correspondence between the zeroes of $\mathfrak{s}(t, \cdot, \mathbf{v})$ and the $J_{t}$-holomorphic gluings of $\mathbf{v}$. Moreover, $\mathfrak{s}(t, \cdot, \mathbf{v})$ is transverse to the zero section of $\mathcal{O}_{\mathbf{u}}^{t}$ (the restriction of $\mathcal{O}_{\mathbf{u}}$ to $\left.\{t\} \times\{*\} \subset \mathcal{B}_{\mathbf{u}}\right)$ for generic $J_{t}$. 
Remark 3.18. Under the assumptions of Remark 3.17, it can be easily computed that

$$
\operatorname{dim} \mathcal{B}_{\mathbf{u}}=\operatorname{virt}-\operatorname{dim}(\mathbf{u})+\operatorname{rank} \mathcal{O}_{\mathbf{u}}
$$

We conclude that if virt-dim(u) $=1$, then generically, for fixed small $t$ and fixed large gluing parameters, there will be finitely many $J_{t}$-gluings, given by zeroes of the obstruction section as a section of $\mathcal{O}_{\mathbf{u}}^{t}$.

Existence of obstruction bundles. In the case where the hypotheses of Lemma 3.14 are not satisfied for a possibly multiply covered curve $u:(\dot{S}, j) \rightarrow H \subseteq W=\mathbb{R} \times M$, I wish to have a criterion for the existence of an obstruction bundle for building configurations containing $u$. I will assume that $u$ is is not too bad in $H$, which includes the case where $u$ is regular (all curves discussed in this article will be leafwise regular). I prove that $u$ is not-too-bad in $\mathbb{R} \times M$, which provides obstruction bundles in $\mathbb{R} \times M$.

As in the proof of Lemma 3.14, consider the splitting

$$
\mathbf{D}_{u}^{N}=\left(\begin{array}{cc}
\mathbf{D}_{u ; T_{H}}^{N} & \mathbf{D}_{u ; N T}^{N} \\
0 & \mathbf{D}_{u ; N_{H}}^{N}
\end{array}\right) .
$$

If the hypotheses of Lemma 3.14 fail, then we have

$$
\text { ind } \mathbf{D}_{u ; N_{H}}^{N} \leq 0 \text {. }
$$

Indeed, this follows from (18), if we assume that $g \geq 1$ or $\# \Gamma_{0}^{-}+\# \Gamma_{1}^{-}+\# \Gamma_{1}^{+}+\# \Gamma_{2}^{-} \geq 2$. By [11], we get a bound

$$
\operatorname{dim} \operatorname{ker} \mathbf{D}_{u ; N_{H}}^{N} \leq K\left(c_{1}\left(N_{H}, u\right), \# \Gamma_{e v e n}\right),
$$

where

$$
2 c_{1}\left(N_{H}, u\right)=\operatorname{ind} \mathbf{D}_{u ; N_{H}}^{N}-2+2 g+\# \Gamma_{\text {even }}=-2\left(\# \Gamma_{0}^{-}+\# \Gamma_{2}^{-}\right) \leq 0
$$

and

$$
K(c, G)=\min \left\{k+l \mid k, l \in \mathbb{Z}^{\geq 0}, k \leq G, 2 k+l>2 c, l \text { even }\right\} .
$$

If $c_{1}\left(N_{H}, u\right)<0$, which is equivalent to either $\# \Gamma_{0}^{-}$or $\# \Gamma_{2}^{-}$being nonzero (which is the case only when $u$ lies in the cylindrical hypersurface over min or max), we have that $\mathbf{D}_{u ; N_{H}}^{N}$ is injective. In the case where $c_{1}\left(N_{H}, u\right)=0$, we obtain

$$
\operatorname{dim} \operatorname{ker} \mathbf{D}_{u ; N_{H}}^{N} \leq 2 .
$$

Case 1: $H$ is noncylindrical. In this case, $\operatorname{dim} \operatorname{ker} \mathbf{D}_{u ; N_{H}}^{N} \geq 1$, since $\partial_{a}$ is then normal to $H$, and the almost complex structure is $\mathbb{R}$-invariant.

Case 1.A: Generic case. If $H$ projects to a flow line joining the maximum to the minimum, all nearby index 2 flow lines are obtained by a pushoff in the $\Sigma$-direction of a normal section $\eta$ which decays asymptotically, and this corresponds to holomorphic pushoffs of $u$ in nearby hypersurfaces. I conclude that

$$
\operatorname{dim} \operatorname{ker} \mathbf{D}_{u ; N_{H}}^{N}=2,
$$


and so

$$
\operatorname{ker} \mathbf{D}_{u}^{N}=\operatorname{ker} \mathbf{D}_{u ; T_{H}}^{N} \oplus\left\langle\partial_{a}, \eta\right\rangle .
$$

Case 1.B: Nongeneric case. In the case of an index 1 flow line, the section $\eta$ is not there, and we have $\operatorname{dim} \operatorname{ker} \mathbf{D}_{u ; N_{H}}^{N}=1$, spanned by the $\mathbb{R}$-direction. We obtain that

$$
\operatorname{ker} \mathbf{D}_{u}^{N}=\operatorname{ker} \mathbf{D}_{u ; T_{H}}^{N} \oplus\left\langle\partial_{a}\right\rangle
$$

For both cases 1.A and 1.B, since the index is only dependent on the moduli space, and I have shown that the dimension of the kernel satisfies the same, I conclude that the dimension of the cokernel depends only on the moduli space. This finishes the noncylindrical case.

Case 2: $H$ is cylindrical. I have already observed that $\mathbf{D}_{u ; N_{H}}^{N}$ is injective for the case where $H$ is cylindrical over the maximum or minimum, but the argument was not enough to cover the case where $H$ is cylindrical over a hyperbolic critical point. I show that the operator $\mathbf{D}_{u ; N_{H}}^{N}$ is injective by a perturbation argument, when $H$ is cylindrical (over any critical point), as follows. For $\epsilon=0, \mathbb{R} \times M_{P}^{ \pm}$is foliated by cylindrical holomorphic hypersurfaces $\mathbb{R} \times Y \times\{p\}$ for any $p \in M_{P}^{ \pm}$. This implies that

$$
\mathbf{D}_{u}^{N}=\mathbf{D}_{u, \epsilon}^{N}=\left(\begin{array}{cc}
\mathbf{D}_{u ; T_{H}}^{N} & \mathbf{D}_{u ; N T}^{N} \\
0 & \mathbf{D}_{u ; N_{H}}^{N}
\end{array}\right) \stackrel{\epsilon \rightarrow 0}{\longrightarrow}\left(\begin{array}{cc}
\mathbf{D}_{u ; T_{H}}^{N} & 0 \\
0 & \bar{\partial}
\end{array}\right) .
$$

The operator $\bar{\partial}$ is injective, since the elements in its kernel are holomorphic sections which decay at the punctures. Since injectivity is an open condition in the usual Fredholm operator topology, it follows that $\mathbf{D}_{u ; N_{H}}^{N}$ is injective for sufficiently small $\epsilon>0$. Therefore,

$$
\operatorname{ker} \mathbf{D}_{u}^{N}=\operatorname{ker} \mathbf{D}_{u ; T_{H}}^{N}
$$

which depends only on the moduli containing $u$, by assumption. This finishes all cases.

Remark 3.19. Note that we have obtained

$$
\operatorname{dim} \operatorname{ker} \mathbf{D}_{u ; T_{H}}^{N}=\operatorname{ind}(\gamma)=\operatorname{ind}\left(p_{+}\right)-\operatorname{ind}\left(p_{-}\right),
$$

if $u$ is contained in a hypersurface $H$ projecting to the flow line $\gamma$, joining the critical points $p_{-}$and $p_{+}$.

Remark 3.20. In Case 2 before, the small value that $\epsilon$ needs to be depends a priori on the curve $u$. While this is perhaps just a technicality, as long as we consider curves $u$ with Morse-Bott asymptotics, of which the positive have bounded total action (for a fixed bound), we can assume that this operator is injective for such a family of curves, since there will be finitely many such moduli spaces.

Observe that in the case of a regular cylinder inside a cylindrical hypersurface, this implies that it is regular in $\mathbb{R} \times M$. This includes the case of a cylinder lying in a cylindrical hypersurface corresponding to a hyperbolic critical point, which is not covered by Lemma 3.14 . 
I have proved the following:

Proposition 3.21 (Cylinders). Every cylinder with two positive ends over a hyperbolic critical point (necessarily the same for both ends) and regular in a noncylindrical hypersurface has an obstruction bundle of rank 1 . We can take $\epsilon>0$ sufficiently small so that cylinders which are regular inside their corresponding hypersurface are also regular in $\mathbb{R} \times M$ for every other case (including all cases where $u$ has a negative end). For cylinders lying in cylindrical hypersurfaces corresponding to a hyperbolic critical point, we can ensure their regularity as long as we consider fixed action bounds on the positive asymptotics, or finite families of moduli spaces.

More generally, we have the following:

Proposition 3.22 (Curves with arbitrary topology). Assume that $u \subseteq H$ is not too bad inside $H$, and the rest of the hypotheses of Lemma 3.14 fail (i.e., either $g>0$ or (16) fails). If $u$ does not lie in a cylindrical hypersurface corresponding to a hyperbolic critical point, then $u$ is not too bad in $\mathbb{R} \times M$. Moreover, for every $T>0$ action threshold, we can choose an $\epsilon>0$ sufficiently small such that every curve $u$ which lies in such a hypersurface and is not too bad inside of it, and with the total action of its positive asymptotics bounded by $T$, is not too bad in $\mathbb{R} \times M$.

In all of the preceding cases, this implies that there exists an obstruction bundle $\mathcal{O}_{\mathbf{u}}$ for gluing $u$ to any building configuration which contains it.

Remark 3.23. It is not hard to show that for small $\epsilon>0$, the rank of $\mathcal{O}_{\mathbf{u}}$ is

$$
\operatorname{rank} \mathcal{O}_{\mathbf{u}}=\operatorname{rank} \mathcal{O}_{u}^{H}-\operatorname{ind} \mathbf{D}_{u ; N_{H}}^{N}+\operatorname{dim} \operatorname{ker} \mathbf{D}_{u ; N_{H}}^{N},
$$

where $\mathcal{O}_{u}^{H}$ is the obstruction bundle of $u$ inside $H$ and the second term is given by (18). Recall from Remark 3.19 that in the case for noncylindrical hypersurfaces $H$, we have $\operatorname{dim} \operatorname{ker} \mathbf{D}_{u ; N_{H}}^{N} \in\{1,2\}$, depending on whether the corresponding flow line is index 1 or 2 ; and for cylindrical hypersurfaces, $\operatorname{dim} \operatorname{ker} \mathbf{D}_{u ; N_{H}}^{N}=0$. It can also be shown that

$$
\text { coker } \mathbf{D}_{u}^{N}=\text { coker } \mathbf{D}_{u ; T_{H}}^{N} \oplus \operatorname{coker} \mathbf{D}_{u ; N_{H}}^{N} \text {. }
$$

Remark 3.24. Given a leafwise not-too-bad curve $u$, if we assume that (19) holds for the leafwise moduli space and the operator $\mathbf{D}_{u ; T_{H}}^{N}$ (e.g., if $u$ is leafwise regular), then the computation of the kernel of the total operator $\mathbf{D}_{u}^{N}$ implies that it also holds in the ambient manifold $\mathbb{R} \times M$.

This finishes the general construction. From now on, I discuss a particular subclass of examples, for which I obtain the results from the introduction.

\section{Nonfillable 5-dimensional model with no Giroux torsion}

For this section, I fix $Y=S T^{*} X$ to be the unit cotangent bundle of a closed hyperbolic surface $X$ with respect to a choice of hyperbolic metric, and $\pi: Y \rightarrow X$ the natural projection. The 1 -form $\alpha_{-}$is the standard Liouville form, and $\alpha_{+}$is a prequantisation contact form (recall the notation introduced in the first paragraph of Section 3.1). This 
means that $\alpha_{+}$is a connection form with curvature $d \alpha_{+}=\pi^{*} \omega$, where $\omega$ is a symplectic form on $X$ representing $c_{1}(Y)=-2+2 g(X)>0$ in $H^{2}(X ; \mathbb{R}) \simeq \mathbb{R}$ when $T^{*} X$ is viewed as a complex line bundle - that is, area $(\omega)=\int_{X} \omega=-\chi(X)$. This example of $Y \times I$ was originally constructed in [11]. I consider the family of 5-dimensional contact manifolds $M=Y \times \Sigma$ constructed in previous sections.

I will dig into the SFT of this class of examples and derive my results. I shall investigate whether these examples have 1-torsion (for any number $k$ of circles in the dividing set, not just $k=1,2$, in which case we already know they do by Theorem 1.3; note that in the case $k=1$, we know the precise algebraic torsion, which is zero). In Section 4.3, I will classify all possible building configurations that can contribute to 1-torsion in the whole SFT algebra, and in Section 4.5 I will prove Theorem 1.5.

\subsection{Curves on symplectisation of prequantisation spaces: Existence and uniqueness}

Consider $\left(Y, \xi=\operatorname{ker} e^{H} \lambda\right)$, a prequantisation space over an integral symplectic base $(X, \omega)$, where $H$ is a Hamiltonian on $X$ and $d \lambda=\pi^{*} \omega$. It is a reasonably standard construction that any choice of $\omega$-compatible complex structure on $X$ naturally lifts to a compatible $J$ on the symplectisation of $Y$, for which there exists a finite-energy foliation $\mathcal{F}_{H}$ by $J$ holomorphic cylinders. These project to $X$ as flow lines of $H$ (with respect to the metric induced by $\omega$ and the almost complex structure on $X$ ), and their asymptotics correspond to circle fibres over the critical points of $H$ (see, e.g., [16, 21]).

Lemma 4.1 (Uniqueness). Let $X$ be a closed surface of genus $g \geq 1$, and let $\omega$ be an integral area form on $X$. Let $\pi: Y \rightarrow X$ denote the prequantisation space over $X$, with a connection (contact) form $\lambda$ whose curvature form satisfies $d \lambda=\pi^{*} \omega$. Then for any choice of $\omega$-compatible complex structure on $X$ lifting to a compatible almost complex structure $J$ on $\mathbb{R} \times Y$, any action threshold $T>0$ and any Morse Hamiltonian $H: X \rightarrow \mathbb{R}$, we can find sufficiently small $\epsilon>0$ such that any genus $g^{\prime}<g$ holomorphic curve on $\left(\mathbb{R} \times Y, e^{\epsilon H} \lambda\right)$ whose positive asymptotics all correspond to critical points of $H$ and have total action bounded by $T$ is a multiple cover of a flow-line cylinder in the finite-energy foliation $\mathcal{F}_{H}$ induced by $H$.

Proof: In the degenerate case $H=0$, the projection $\mathbb{R} \times Y \rightarrow X$ is holomorphic, and every Reeb orbit in $Y$ is a multiple of the $S^{1}$-fibre. So a curve as in the statement induces a holomorphic map into $X$, defined on a closed curve of genus $g^{\prime}$. By holomorphicity, it has nonnegative degree, and it has zero degree if and only if it is constant. If it has positive degree, by Poincaré duality it induces an injection in cohomology - that is, we get an injective map $\mathbb{R}^{2 g} \hookrightarrow \mathbb{R}^{2 g^{\prime}}$. But this cannot happen if $g^{\prime}<g$. I conclude that it has vanishing degree, and so the curve is a cover of a the trivial cylinder.

In the nondegenerate case where $H$ is a small but nonidentically zero Morse perturbation, one can use holomorphic cascades (in the sense of [1]) to reduce to the degenerate case, or alternatively the results from [11] (see the proof of [16, Lemma 6.3]). 


\subsection{Some remarks and useful facts}

Recall that ( $Y=S T^{*} X, \alpha_{-}$) is the unit cotangent bundle of a closed hyperbolic surface, and $\alpha_{-}$is the standard Liouville form. I shall need the following facts about closed $\alpha_{-}-$ orbits (e.g., $[4,18,16])$ :

- They project to $X$ as closed hyperbolic geodesics.

- They are nondegenerate.

- Their Conley-Zehnder indices vanish in a natural trivialisation.

Consider the symplectic form $d \alpha$ on $Y \times I$, where the Liouville form $\alpha$ can be taken as described in the first paragraph of Section 3.1 (cf. [11]). Take $J_{0}$, a cylindrical $d \alpha-$ compatible almost complex structure in the completion $W_{0}=\widehat{Y \times I}$. Recall that in Section 3.3 we defined an almost complex structure $J_{\epsilon}^{1}$ in $\mathbb{R} \times M$, which is compatible with the SHS $\mathcal{H}_{\epsilon}^{1}$ and for which we have a foliation by holomorphic hypersurfaces $\mathcal{F}$. By a generic perturbation of $J_{0}$ (and hence of $J_{\epsilon}^{1}$ along the hypersurfaces in $\mathcal{F}$ ), using [16, Thm. 4.4.3] we can assume the following:

(A) Every somewhere injective holomorphic curve $u$ in the completion $W_{0}=\widehat{Y \times I}$ which intersects the main level (recall Remark 3.13) is regular, and therefore satisfies ind $W_{0}(u) \geq 0$ (where this denotes the index computed in $W_{0}$ ).

Using $c_{1}\left(\xi_{ \pm}\right)=0$, from Remark 3.5 we obtain the following:

(B) $c_{1}\left(\xi_{\epsilon}^{1}\right)=0$.

Observe that since closed Reeb orbits for $\alpha_{-}$project down to closed hyperbolic geodesics, they are noncontractible, and Reeb orbits for $\alpha_{+}$project to points. This implies the following fact:

(C) There are no holomorphic cylinders in my model crossing sides of the dividing set.

This does not happen for the 3-dimensional cases in [11], where the hypersurfaces are cylinders.

Observe that we are allowed to take a generic almost complex structure $J_{-}$on $(\mathbb{R} \times$ $\left.Y_{-}, d\left(e^{a} \alpha_{-}\right)\right)$and use it in the construction of the model. Since the index of every cylinder is necessarily zero, every multiply covered cylinder is unbranched and multiple covers of trivial cylinders are again trivial, we obtain the following:

(D) Every holomorphic cylinder in $\left(\mathbb{R} \times Y, d\left(e^{a} \alpha_{-}\right)\right)$is necessarily trivial. And every holomorphic curve of index zero is necessarily a trivial cylinder.

Recall that for a given free homotopy class of simple closed curves in a hyperbolic surface $X$, there is a unique geodesic representative. This implies that if two Reeb orbits in $\left(Y, \alpha_{-}\right)$are joined by a holomorphic cylinder in $W_{0}$ with two positive ends (which by $\mathrm{D}$ is necessarily contained in the main level $W_{0}$ ), they correspond to the same geodesic, but with different orientation. In particular, they are disjoint Reeb orbits. We conclude the following:

(E) In $W_{0}$, every holomorphic cylinder with two positive ends on a left upper level joins disjoint Reeb orbits. 


\subsection{Investigating 1-torsion}

In this section, I will study the portion of the SFT differential of the contact manifold ( $\left.M=S T^{*} X \times \Sigma, \xi\right)$ which has the potential to yield 1-torsion, where I denote by $\xi$ the isotopy class of contact structures on $M$ that I have defined in previous sections. Recall from the introduction that we have a power series expansion $\mathbf{D}_{S F T}=\sum_{k \geq 1} D_{k} \hbar^{k-1}$ of said differential, where $D_{k}$ is a differential operator of order $\leq k$. This operator is defined by counting holomorphic curves in $\mathbb{R} \times M$ with $\left|\Gamma^{+}\right|+g=k$, where $\Gamma^{+}$is the set of positive punctures and $g$ is the genus. Here I consider the untwisted version of the SFT, where I do not keep track of homology classes. I will compute the projection of the operators $D_{1}$ and $D_{2}$ to the base field $\mathbb{R}$, but consider their actions on Reeb orbits only up to a large action threshold. In other words, forget the $q$-variables from these operators, so that we only consider curves with no negative ends. While the projection of $D_{1}$ to $\mathbb{R}$ vanishes identically (there are no holomorphic disks), the computation for $D_{2}$ is rather involved. According to [16, Lemma 4.23] (a generalisation of [11, Lemma 4.15]), for $N=1$ - where $N$ is as in the statement of that lemma - if all holomorphic building configurations with arithmetic genus $g$, no negative ends and $r$ positive ends satisfying $g+r \leq 2$ come in cancelling pairs (and so the $\mathbb{R}$-components of $D_{1}$ and $D_{2}$ are zero), then there is no 1-torsion. However, the result is rather unexpected: among precisely 35 possibilities of such building configurations, there is only one, the sporadic configuration, which does not cancel. This mean that the only way to obtain 1-torsion (below the action threshold) is to differentiate the asymptotics of this configuration, since the differential of all other low-action orbits has (at most) $q$ variables in the $D_{2}$ coefficient appearing in front of $\hbar$. While I cannot prove rigorously that 1-torsion indeed arises, in the next section I provide a heuristic argument as to why I expect this to be true. While I originally expected that the classification of these buildings would show that 1-torsion does not arise, I will use knowledge of cylinder configurations (the fact that they cancel in pairs) to prove Theorem 1.5.

Setup. Denote by $H_{X}$ a choice of a Morse function satisfying the Morse-Smale condition on $X$. Choose a (nongeneric) cylindrical almost complex structure $J_{+}$on the symplectisation of $\left(Y, \alpha_{+}=e^{\epsilon^{\prime} H_{X}} \lambda\right)$, coming from a lift of a complex structure on $X$, inducing a foliation $\mathcal{F}_{X}$ of $\mathbb{R} \times Y$ by $H_{X}$-flow-line cylinders (as described in Section 4.1). Here, choose $\epsilon^{\prime}>0$ small enough so that Lemma 4.1 holds for $T=T_{\epsilon^{\prime}}$. Denote by $\gamma_{p ; q}$ the simply covered Reeb orbit in $M$ corresponding to $(p, q) \in \operatorname{crit}\left(h_{+}\right) \times \operatorname{crit}\left(H_{X}\right)$ (recall the Morse functions $h_{ \pm}$introduced in Section 3.1). Given a holomorphic curve (or building) $u$ in $\mathbb{R} \times M$ with asymptotics corresponding to critical points of $h_{ \pm}$, for either one or both of the Morse functions, and then lying in a hypersurface of the foliation $\mathcal{F}$, I will view it as a punctured curve in $W_{0}$ when convenient (recall Remarks 3.7 and 3.8). I will denote by $\operatorname{ind}_{M}(u)$ (resp. ind $W_{0}(u)$ ) its Fredholm index when viewed as a curve in $\mathbb{R} \times M$ (resp. in $\left.W_{0}\right)$. By $(\mathrm{B})$, we have

$$
\begin{gathered}
\operatorname{ind}_{M}(u)=\left(\mu_{C Z}^{\tau}\right)_{M}(u) \\
\operatorname{ind}_{W_{0}}(u)=-\chi(u)+\left(\mu_{C Z}^{\tau}\right)_{W_{0}}(u) .
\end{gathered}
$$


Note that the absence of the Euler characteristic term in the first formula is because $\mathbb{R} \times M$ is 6-dimensional (whereas $W_{0}$ is 4 -dimensional). By Proposition 3.10, given $l \leq N_{\epsilon}$, then for orbits $\gamma_{p ; q}^{l}$ for hyperbolic $p$, we have $\left(\mu_{C Z}^{\tau}\right)_{M}\left(\gamma_{p ; q}^{l}\right)=\left(\mu_{C Z}^{\tau}\right)_{W_{0}}\left(\gamma_{p ; q}^{l}\right)=\operatorname{ind}(q)-1$; and for orbits $\gamma_{p ; q}^{l}$, for $p$ the maximum or minimum, we have $\left(\mu_{C Z}^{\tau}\right)_{M}\left(\gamma_{p ; q}^{l}\right)=\left(\mu_{C Z}^{\tau}\right)_{W_{0}}\left(\gamma_{p ; q}^{l}\right)+$ $1=\operatorname{ind}(q)$.

Recall that $k$ is the number of circles in the dividing set. For $k \leq 2$, we already know that the model has 1 -torsion (by Theorem 1.3), so set $k \geq 3$. Denote by $\overline{\mathcal{M}}_{g, r}^{1}\left(J_{\epsilon}^{1} ; T_{\epsilon}\right.$ ) the moduli space of all translation classes of index 1 connected $J_{\epsilon}^{1}$-holomorphic buildings in $\mathbb{R} \times M$ with arithmetic genus $g$, no negative ends and $r$ positive ends approaching orbits whose periods add up to less than $T_{\epsilon}$. Elements in this moduli space are the buildings that potentially glue (in general, after an abstract polyfold perturbation; or a generic perturbation of $J$, in the case where the element has an obstruction bundle) to curves which might contribute to the projection to the ground field $\mathbb{R}$ of the SFT differential. I will prove the existence of a choice of coherent orientations such that elements in $\overline{\mathcal{M}}_{g, r}^{1}\left(J_{\epsilon}^{1} ; T_{\epsilon}\right)$, whenever $g+r \leq 2$, cancel in pairs (after introducing, in sequence, a contact perturbation of the stable Hamiltonian structure; a nondegenerate perturbation of the contact form; and a generic perturbation of $J$ ), except for a single sporadic building configuration. This unusual configuration consists of a single-level punctured torus, and I will discuss it in the next section.

Classification. If $\mathbf{u}$ is an element of $\overline{\mathcal{M}}_{g, r}^{1}\left(J_{\epsilon}^{1} ; T_{\epsilon}\right)$ with $g+r \leq 2$, since $r \geq 1$ by exactness, then it can only glue to one of the following:

- Case 1. A plane with one positive end.

- Case 2. A cylinder with two positive ends.

- Case 3. A 1-punctured torus with one positive end.

Case 1 is ruled out, since Reeb orbits in $Y$ are noncontractible (for both the prequantisation form and the standard Liouville form), as are the Reeb orbits in $Y \times \mathcal{U}$. The other orbits are ruled out by the definition of $T_{\epsilon}$ and $\overline{\mathcal{M}}_{g, r}^{1}\left(J_{\epsilon}^{1} ; T_{\epsilon}\right)$. This means that there is no 0-torsion, so this already shows that the model is tight (we already knew this from [16], since both $\left(Y, \alpha_{ \pm}\right)$are hypertight).

So I take up the other two cases. Case 2 is the only one which is relevant for Theorem 1.5 and Corollary 1.6, so I will not include details for case 3, which are rather involved. I first observe that the Reeb orbits in the asymptotics of every floor of $\mathbf{u}$ are of the form $\gamma_{p ; q}^{l}$, for some $l \leq N_{\epsilon}$ (for $N_{\epsilon}$ as in Proposition 3.10). This is certainly the case for asymptotics outside of the cylindrical region, by the action restriction given by $T_{\epsilon}$. Since Reeb orbits like these project to $\Sigma$ as points, the projection of $\mathbf{u}$ to $\Sigma$ is a cobordism between the sum of the positive asymptotics of the top floor of $\mathbf{u}$ lying in $Y \times \mathcal{U}$ (which are positive multiples of the dividing circles) and zero. On the other hand, since $\mathbf{u}$ has at most two positive asymptotics, there is at least one component of $Y \times \mathcal{U}$ which does not contain asymptotics of $\mathbf{u}$ (here use $k \geq 3$ ). But then, one can find a simple closed curve which passes through this component and has a nonzero intersection number with the sum of these asymptotics, which is absurd (see Figure 6). By induction, removing the top floor at each step, we obtain the claim. 


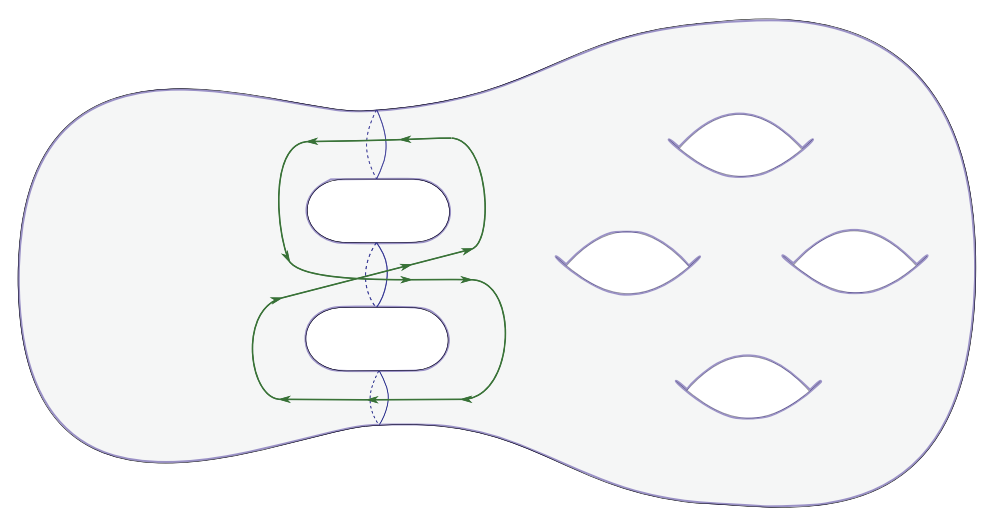

Figure 6. A simple closed curve in $\Sigma$ with nonzero intersection number with the projection of the positive asymptotics which lie in $Y \times \mathcal{U}$ of a hypothetical building $\mathbf{u} \in \overline{\mathcal{M}}_{g, r}^{1}\left(J_{\epsilon}^{1} ; T_{\epsilon}\right)$ with $g+r \leq 2$, containing such. It follows that no such $\mathbf{u}$ exists.

I can then appeal to Proposition 3.12, which yields the fact that each component of $\mathbf{u}$ lies in one of the holomorphic hypersurfaces of the foliation, so that $\mathbf{u}$ can be viewed as a building inside $W_{0}=\widehat{Y \times I}$.

\section{Case 2. A cylinder with two positive ends.}

Since $\mathbf{u}$ glues to a cylinder with no negative ends, and since the Reeb orbits $\gamma_{p ; q}$ are noncontractible, then it can be shown that $\mathbf{u}$ can only consist of a cylinder with two positive ends in the bottom floor, together with two chains of cylinders on top of its ends (see Figure 7).

By $(\mathrm{C})$, no component of $\mathbf{u}$, all of which are cylinders, can have asymptotics in different sides. Also, recall that we can assume that $\mathbf{u}$ is a stable building, so that it does not have levels consisting only of trivial cylinders.

We see that $\mathbf{u}$ cannot consist solely of right upper-level components (corresponding to the prequantisation space), since its bottom level would be a cylinder with two positive ends and then cannot be a cover of a flow-line cylinder, which contradicts Lemma 4.1. We also see that it cannot consist solely of left upper-level components, since its bottom level cannot be a trivial cylinder (it has no negative ends), which contradicts observation(D).

Then $\mathbf{u}$ has a nontrivial component in the main level, which I call $u_{0}$.

Case 2.A. Both asymptotics of $u_{0}$ lie on a left level. Label by hyp or min the Reeb orbits appearing in $\mathbf{u}$, according to whether they lie over a hyperbolic point or the minimum. Since $u_{0}$ lies in a hypersurface, its two positive asymptotics necessarily have the same label. Thus, for each string, the associated ordered sequence of labels can only look like (hyp, .., hyp, min, .., min). The number of hyps or mins may be zero, but not both (see Figure 8). Observation (D) implies that all the upper components correspond to trivial cylinders (under Remark 3.8). We then see that the only possibility for $\mathbf{u}$ is the one depicted in Figure 8, since all others will have index different from 1. 


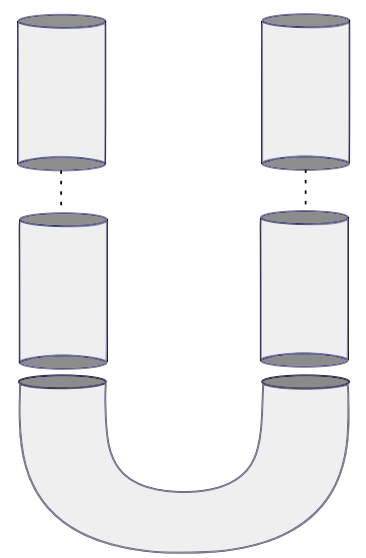

Figure 7. The only possible a priori combinatorics for $u$ in the case $g=0, r=2$.

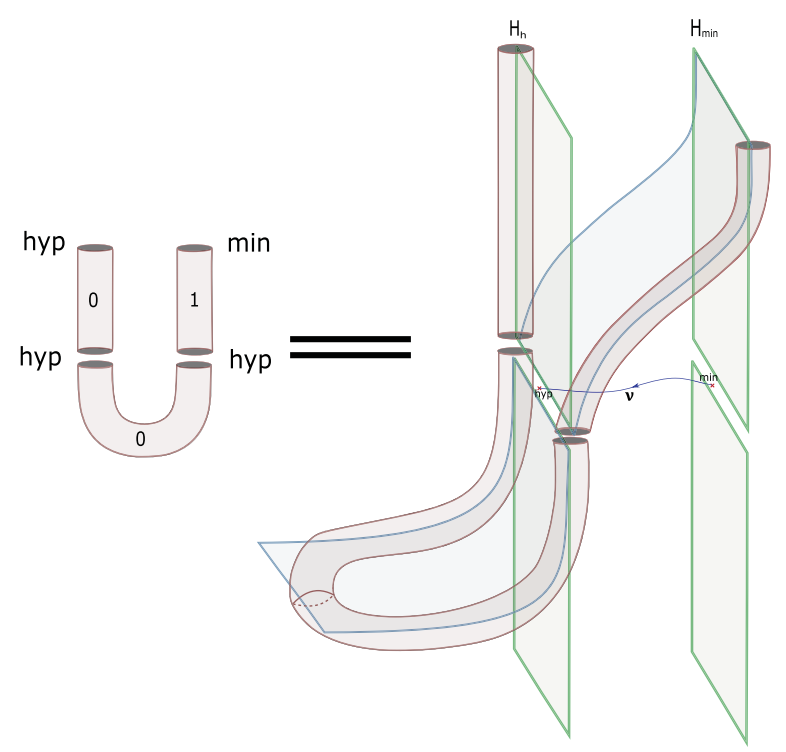

Figure 8. A possible configuration for the index 1 building $u$ for the case $g=0, r=2$.

Observe that $u_{0}$ has only hyperbolic asymptotics, and if it is multiply covered, it is necessarily unbranched. Since $J_{0}$ is generic, Theorem A.3 implies that $u_{0}$ is regular in $W_{0}$, and the same is true for its upper components, which are trivial. By Proposition 3.21, we obtain an obstruction bundle of rank 1 for this configuration.

This configuration has a cancelling 'evil twin', obtained by replacing the index 1 upper component, which lies over an index 1 Morse flow line $\gamma$ connecting hyp to min, with the index 1 cylinder lying over the flow line $\bar{\gamma}$ (the unique other flow line connecting hyp to min). Taking the coherent orientation to be compatible with the Morse orientation (as 
in [11, Rem. 4.14]), the algebraic counts of zeroes of the obstruction sections associated to the twin configurations will cancel out after introducing a generic perturbation (i.e., a geometric perturbation of $J$ ).

One can check that the associated obstruction bundles satisfy the properties of Remark 3.17. Indeed, observe that the moduli space of $u_{0}$ is just a copy of $\mathbb{R}$ corresponding to its $\mathbb{R}$-translations, since it is otherwise geometrically isolated (in both the hypersurface and the $\Sigma$ directions). This includes the case where $u_{0}$ is multiply covered, since it is a unbranched cover. The other components are a trivial cylinder, which is not included in the obstruction bundle, and a regular index 1 cylinder, which is rigid after dividing by $\mathbb{R}$. If $u_{0}$ is multiply covered with degree $d$, then so is the index 1 component. We then see that its obstruction bundle is the trivial bundle

$$
\mathcal{O}_{\mathbf{u}}=\mathbb{R} \times[0,1] \times[R,+\infty) \rightarrow[0,1] \times[R,+\infty)
$$

where $R$ is a large gluing parameter. This implies that conditions i and ii in Remark 3.17 are satisfied.

\section{Case 2.B. Both asymptotics of $u_{0}$ lie on a right level.}

Every component in the upper levels of $\mathbf{u}$ is either contained in a holomorphic hypersurface $H_{a}^{v}$ corresponding to an index 1 Morse flow line $v$ connecting a hyperbolic point hyp $\in \Sigma_{+}$to the maximum $\max \in \Sigma_{+}$or contained in a hypersurface $\mathbb{R} \times H_{\text {hyp }}$ or $\mathbb{R} \times H_{\max }$ lying over either hyp or max. Moreover, Lemma 4.1 implies that when $\mathbf{u}$ is viewed as lying inside $W_{0}$, they are (necessarily unbranched) covers of flow-line cylinders. Denote by $q_{1}, q_{2} \in \operatorname{crit}\left(H_{X}\right) \subseteq X$ the critical points corresponding to the two positive asymptotics of $u_{0}$. Label by (hyp; $\left.\operatorname{ind}_{q}\left(H_{X}\right)\right)$ or $\left(\max ; \operatorname{ind}_{q}\left(H_{X}\right)\right)$ the Reeb orbits appearing in $u$, according to whether they lie over a hyperbolic point or the maximum, and where $q \in \operatorname{crit}\left(H_{X}\right)$ is the corresponding critical point. Again, since $u_{0}$ lies in a hypersurface, the first component of the labels of its two positive asymptotics necessarily agree.

Observe that if $v$ denotes a right upper-level component of $\mathbf{u}$, we have

$$
1=\operatorname{ind}_{M}(\mathbf{u})=\operatorname{ind}_{M}\left(u_{0}\right)+\sum_{v} \operatorname{ind}_{M}(v) \geq \operatorname{ind}_{M}(v) \geq \operatorname{ind}_{W_{0}}(v) \geq 0 .
$$

Denote by $u_{0}^{\prime}$ the somewhere injective curve underlying $u_{0}$, which, since Reeb orbits are noncontractible, is a cylinder over which $u_{0}$ is unbranched and satisfies $\operatorname{ind}_{W_{0}}\left(u_{0}^{\prime}\right)=$ ind $_{W_{0}}\left(u_{0}\right)$. By $(\mathrm{A})$, we have that

$$
0 \leq \operatorname{ind}_{W_{0}}\left(u_{0}^{\prime}\right)=\operatorname{ind}_{W_{0}}\left(u_{0}\right)=\operatorname{ind}_{p}\left(H_{X}\right)-1+\operatorname{ind}_{q}\left(H_{X}\right)-1 \leq \operatorname{ind}_{M}(u)=1,
$$

so that

$$
3 \geq \operatorname{ind}_{p}\left(H_{X}\right)+\operatorname{ind}_{q}\left(H_{X}\right) \geq 2 .
$$

If the two labels of the positive ends of $u_{0}$ are max, then its index in $M$ is

$$
\operatorname{ind}_{M}\left(u_{0}\right)=\operatorname{ind}_{p}\left(H_{X}\right)+\operatorname{ind}_{q}\left(H_{X}\right) \geq 2 .
$$




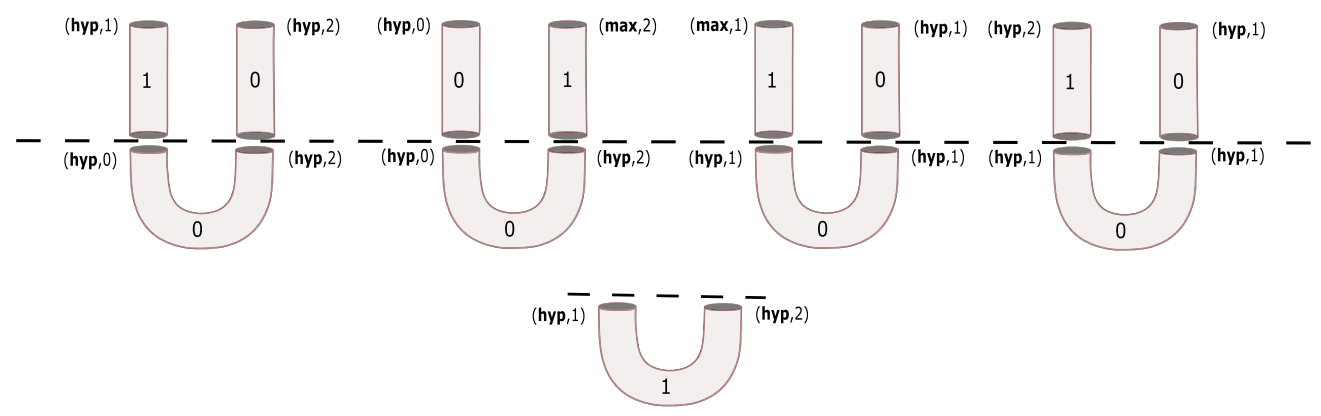

Figure 9. All five possible configurations with positive ends on a right upper level (up to obvious symmetries). The dotted lines separate the main levels from the upper levels. The upper-level components correspond to unbranched covers of flow-line cylinders over $X$, which are regular inside their corresponding hypersurface.

Since $1=\operatorname{ind}_{M}(u) \geq \operatorname{ind}_{M}\left(u_{0}\right) \geq 2$, we get a contradiction. Then both asymptotics of $u_{0}$ have hyp as the first component of their label, which in particular implies ind $W_{0}\left(u_{0}\right)=$ $\operatorname{ind}_{M}\left(u_{0}\right)$.

Assume $\operatorname{ind}_{p}\left(H_{X}\right)+\operatorname{ind}_{q}\left(H_{X}\right)=2$, so that $u_{0}$ has index zero in $W_{0}$. In the case that $\operatorname{~ind~}_{p}\left(H_{X}\right)$, ind $\left.q_{q}\left(H_{X}\right)\right)=(0,2)$, the automatic transversality criterion in [11] implies that it is regular in $W_{0}$. If $\operatorname{ind}_{p}\left(H_{X}\right)$, ind $\left.\operatorname{in}_{q}\left(H_{X}\right)\right)=(1,1)$, then we use Theorem A.3 to conclude again that $u_{0}$ is regular in $W_{0}$. The only possibilities for $\mathbf{u}$ are shown in Figure 9; all of them have a cancelling 'evil twin' in the Morse theory sense, and an associated obstruction bundle of rank 1 .

We have only one case left: $\left.\operatorname{ind}_{p}\left(H_{X}\right), \operatorname{ind}_{q}\left(H_{X}\right)\right)=(1,2)$ (and both labels hyp). Then ind $_{W_{0}}(u)=\operatorname{ind}_{M}\left(u_{0}\right)=1$, and $u=u_{0}$ is nonbroken, that is, has only one level, with a nontrivial main level. The bottom component $u_{0}$ is regular inside its hypersurface, as can be checked via automatic transversality. The resulting configuration, depicted in Figure 9, also has a rank 1 obstruction bundle and a cancelling evil twin, since it lies over an index 1 flow line connecting a hyperbolic point in $\Sigma_{+}$to the minimum in $\Sigma_{-}$.

We can also study the obstruction bundles for all the configurations in Figure 9, and see that we are in the situation explained in Remark 3.17. For the ones with two floors, the obstruction bundle is exactly the same as that of the configuration in Figure 8 - that is, the trivial $\mathbb{R}$ bundle over $[0,1] \times[R,+\infty)$, for large gluing parameter $R \gg 0$. For the unique configuration with a single floor, let us assume the worst case scenario: that $u_{0}$ is an unbranched cover of degree $d$. Then we see that its moduli space is 2-dimensional, of the form $\mathcal{M}_{0} \cong \mathbb{R} \times \mathcal{M}_{H}$, where the $\mathbb{R}$-component corresponds to its $\mathbb{R}$-shifts and $\mathcal{M}_{H}$ denotes its moduli space inside the hypersurface $H$ containing $u_{0}$. Therefore, $\mathcal{M}_{0} \backslash \mathbb{R} \cong \mathcal{M}_{H}$, and this is at worst a 1-dimensional orbifold. These come only in two types: a circle or an interval. I conclude that $\mathcal{O}_{\mathbf{u}}$ is (at worst) an $\mathbb{R}$-bundle over a cylinder $[0,1] \times S^{1}$ or a square $[0,1] \times A$ for some interval $A$. This implies that the conditions of Remark 3.17 are satisfied.

I conclude that after perturbing to generic $J$, the count of gluings of the configurations considered is zero, and finishes case 2 . 


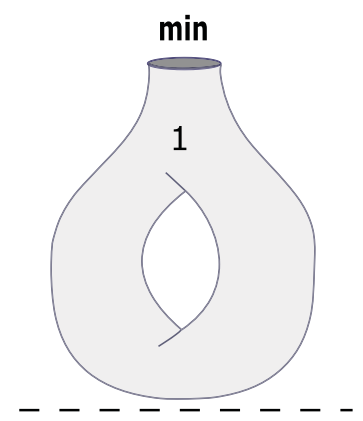

Figure 10. The sporadic configuration.

\section{Case 3. 1-punctured torus with one positive end.}

I will not give details of the classification, since I shall not need it for my purposes. There are 29 such configurations, all of which have obstruction bundles. Moreover, all have mirror cancelling pairs, except one of them, depicted in Figure 10. See [16].

\subsection{The sporadic configuration}

In this section, I address Conjecture 1.7. Let $u$ be the sporadic configuration depicted in Figure 10. Observe first that $u$ is in fact regular in $\mathbb{R} \times M$. Indeed, since it cannot be a cover of a plane, it is somewhere injective, and so regular in the hypersurface $H:=\mathbb{R} \times H_{\min }$. Equation (18) implies that ind $\mathbf{D}_{u ; N_{H}}^{N}=0$, and since $\mathbf{D}_{u ; N_{H}}^{N}$ is injective ( $H$ is cylindrical), we have that it is surjective. By (26), I deduce the claim.

Some string topology. Recall the definition of Goldman-Turaev string bracket and cobracket operations in the case of a hyperbolic surface. This is the starting point of Chas and Sullivan's string topology [2]. I follow the exposition of [16], which builds on [4].

Let $X$ be a closed surface of genus at least 2 . The set of nontrivial free homotopy classes of loops (i.e., a conjugacy class of the fundamental group) is countable. Choose some ordering on this set, label by $[i]$ the $i$ th one and make the convention that $[-i]$ denotes a change in orientation of the corresponding geodesic. Let $V$ denote the vector space generated by the free homotopy classes of noncontractible loops on $X$. We define the string cobracket$$
\Delta: V \rightarrow V \otimes V
$$

as follows. Represent $[i]$ by a string $s$ in general position, so that it has finite selfintersections $x_{1}, \ldots, x_{k}$. At any $x_{j}$ there are two directions in the string, which we orient by the orientation on $X$. We can resolve the string $s$ at each self-intersection $x_{j}$, obtaining two new strings $s_{1}^{j}$ and $s_{2}^{j}$. For instance, $s_{1}^{j}$ is obtained by following the first direction out of $x_{j}$ and taking the piece of $s$ connecting $x_{j}$ back to itself, and similarly for $s_{2}^{j}$, following 
the second direction. Define

$$
\Delta([i])=\sum_{j=1}^{k}\left[s_{1}^{j}\right] \otimes\left[s_{2}^{j}\right]-\left[s_{2}^{j}\right] \otimes\left[s_{1}^{j}\right] .
$$

This cobracket is a Lie cobracket - that is, it is bilinear and co-antisymmetric and satisfies the co-Jacobi identity. Similarly, define the string bracket

$$
\nabla: V \times V \rightarrow V
$$

which is a Lie bracket (see [16]).

In this notation, we have the coefficients $A_{j, k}^{i}$ for $\Delta$, defined by

$$
\Delta([i])=\sum_{j, k} A_{j, k}^{i}[j] \otimes[k] .
$$

On the SFT side, denote by $d^{i}$ the SFT-count of sporadic configurations inside $\mathbb{R} \times S T^{*} X$ whose positive asymptotic corresponds to $[i]$. Cieliebak and Latschev show, by looking at the boundary of index 1 moduli spaces of holomorphic curves with Lagrangian boundary components in $T^{*} X$, the existence of linear relationships between the count of index 1 curves in $\mathbb{R} \times S T^{*} X$ and the coefficients of these string operations. This allows them to compute the full SFT Hamiltonians for $T^{*} X$ and $\mathbb{R} \times S T^{*} X$. In the case of 1-punctured tori, we have the following [16, Thm. 2.2.1]:

$$
d^{i}=\sum_{j} A_{j,-j}^{i} \in \mathbb{Z} .
$$

Relationship to 1-torsion [4, Figure 17] gives an example of a closed geodesic [i] for which $d^{i}$ is nonzero. Denote by $q_{i}$ the corresponding SFT generator in $\mathbb{R} \times M$. Since I have shown that every sporadic configuration is leafwise regular, we have

$$
\mathbf{D}_{S F T}\left(\frac{q_{i}}{d^{i}}\right)=\hbar+\mathcal{O}\left(\hbar^{2}\right) .
$$

Observe that I have used the fact that there are no holomorphic disks.

Now all the other contributions to the previous differential come from potential index 1 building nodal configurations $\mathbf{u}$ with only one positive end approaching $[i]$, which glue to curves after introducing an abstract perturbation. If such a building is entirely contained in $\mathbb{R} \times H_{\text {min }}$, then

$$
1=\operatorname{ind}_{M}(\mathbf{u})=1-\# \Gamma^{-}(\mathbf{u}),
$$

from which we obtain that $\# \Gamma^{-}(\mathbf{u})=0$. And if $\mathbf{u}$ is not contained in this cylindrical hypersurface, we have two cases: either its (unique) top-level curve $u$ projects to an index 2 flow line connecting the minimum to the maximum or it lies over an index 1 flow line. In the first case, the top-level curve $u$ cannot have any other ends, since the hypersurface containing it has only positive ends, but this cannot happen, since it only has one positive end (over the minimum) and Reeb orbits are noncontractible. In the second case, $u$ has a twin over the opposite index 1 flow line. However, we do not know whether the latter building configurations have associated obstruction bundles, and indeed there are a priori 
many of them to classify (they can have any number of negative punctures, as well as any positive genus, always having Fredholm index 1 in $M$ ).

In any case, this is strong geometric evidence for the following:

Conjecture 4.2. Any building configuration that can contribute to the differential of $q_{i}$ and does not lie in $\mathbb{R} \times H_{\text {min }}$ cancels its twin configuration.

If this conjecture were true, then we would have that the term $\mathcal{O}\left(\hbar^{2}\right)$ does not have any $q$ variables. Since each term of the form $1+\mathcal{O}(\hbar)$ not involving $q$ variables can be inverted as a power series in $\hbar$, we would obtain

$$
\mathbf{D}_{S F T}\left(\frac{q_{i}}{d^{i}}(1+\mathcal{O}(\hbar))^{-1}\right)=\hbar,
$$

and so our 5-dimensional model would have 1-torsion (which would prove Conjecture 1.7).

Observe that this absence of $q$ variables is a purely 5-dimensional phenomenon, in the following sense: since $\operatorname{ind}_{W_{0}}(u)=-\chi(u)$ for a curve $u$ inside $H=\mathbb{R} \times H_{\text {min }}$, the only possible contributions for the differential of $q_{i}$ inside $H$ come from our sporadic configuration, as well as 3-punctured spheres with one positive end, at $[i]$, and two negative ones, at $[j]$ and $[k]$, say. However, the latter configurations have index -1 in $\mathbb{R} \times M$, so do not contribute to $\mathbf{D}_{S F T} q_{i}$ in the ambient manifold. Moreover, from [4] and [16] we have that the SFT count of such curves inside $H$, which we denote $a_{j, k}^{i}$, coincides with the string coefficients $a_{j, k}^{i}=A_{j, k}^{i}$. Observe that we know from (27) that there is at least one $j$ for which $A_{j,-j}^{i}$ is nonzero. So indeed, we can find 3-punctured spheres in $H$ which contribute nontrivially to the SFT count in $H$ but do not in $\mathbb{R} \times M$.

\subsection{Non-SFT proof of Theorem 1.5}

In this section, I prove Theorem 1.5. Recall the definition of a Giroux $2 \pi$-torsion domain: given a Liouville pair $\left(\alpha_{+}, \alpha_{-}\right)$on a closed manifold $Y^{2 n-1}$, this is the contact manifold $\left(G T, \xi_{G T}\right):=\left(Y \times[0,2 \pi] \times S^{1}, \operatorname{ker} \lambda_{G T}\right)$, where

$$
\lambda_{G T}=\frac{1+\cos (r)}{2} \alpha_{+}+\frac{1-\cos (r)}{2} \alpha_{-}+\sin (r) d \theta
$$

and the coordinates are $(r, \theta) \in[0,2 \pi] \times S^{1}$.

This contact manifold carries a suitable notion of an SOBD. These SOBDs, which I call Giroux $S O B D$, were described in detail in [18, 16], for more general contact manifolds obtained by gluing together collections of 'Giroux domains'. In the case of Giroux $2 \pi$-torsion domains, the SOBD structure is obtained by declaring small $\delta$-collar neighbourhoods of the slices $\{r \in\{0, \pi, 2 \pi\}\}$ to be the 'paper' components, and their complement the 'spine'. The paper components are then trivial fibrations over $Y$ with fibres (the pages) which are cylinders $[-\delta, \delta] \times S^{1}$, and the spine components are trivial $S^{1}$-fibrations over a Liouville domain of the form $Y \times I$ for some interval $I$. In particular, the model A construction of contact structures of $[18,16]$ can be used. We obtain a contact form $\Lambda_{G T}$ which lies in the isotopy class of $\lambda_{G T}$, together with a finite-energy foliation by holomorphic curves of $\mathbb{R} \times G T$, such that the cylindrical pages lift as holomorphic cylinders with two positive ends asymptotic to Reeb orbits corresponding to critical points 
of a Morse function in $Y \times I$. Moreover, we have a uniqueness theorem for punctured holomorphic curves in $\mathbb{R} \times G T$, which states that any other holomorphic curve with asymptotics which are simply covered and correspond to critical points has to be a reparametrisation of a holomorphic cylinder lifting a page (see [16, Theorem 3.9] and its adaptation in the proof of Theorem 5.2 also in that thesis; it involves, among other things, using energy estimates to reduce the problem to dimension 4, where intersection theory may be used). If we choose a critical point of index 1 and a critical point of index $2 n$, there exists a unique ( $\mathbb{R}$-translation class of a) regular index 1 holomorphic cylinder in the finite-energy foliation with asymptotics corresponding to these critical points, which I call $u_{0}$. (See $[16]$ for the full details.)

We are now ready to obtain an application from knowledge of the SFT algebra of $S T^{*} X \times \Sigma$. Recall that I distinguish between model A (constructed in [16]) and model B (constructed in this article), which give isotopic contact structures.

\subsection{Proof of Theorem 1.5}

Let $\left(M_{0}, \xi_{0}\right)$ be a 5-dimensional contact manifold with Giroux torsion, and let $i$ : $\left(G T, \xi_{G T}\right) \hookrightarrow\left(M_{0}, \xi_{0}\right)$ be a contact embedding. Consider the 5 -dimensional model $(M=$ $\left.S T^{*} X \times \Sigma, \xi\right)$, with $k \geq 3$, where $\xi=\operatorname{ker} \Lambda_{\epsilon}$ is viewed as the contact structure induced by a model $\mathrm{B}$ contact form $\Lambda_{\epsilon}$ (called $\xi_{k}$ in the introduction). The $\epsilon$-parameter does not change the isotopy class, and I will choose it suitably later. We can take a contact form $\Lambda_{0}$ for $\xi_{0}$, such that it coincides with a model A contact form $\Lambda_{G T}$ on $i(G T)$ as already described. Assume that $(W, d \lambda)$ is an exact cobordism with convex end $\left(M_{0}, \xi_{0}\right)$ and concave end $(M, \xi)$. Let $J_{1}$ be a model B almost complex structure on the negative half-symplectisation of $(M, \xi)$ (which is compatible with $\Lambda_{\epsilon}$ ). Also let $J_{0}$ be a $\Lambda_{0^{-}}$ compatible almost complex structure on the positive half-symplectisation of $\left(M_{0}, \xi_{0}\right)$, which coincides with a model A almost complex structure on $i(G T)$ for which we have the finite-energy foliation by holomorphic curves of $\mathbb{R} \times i(G T)$ already described.

By attaching small trivial symplectic cobordisms to $W$, we can assume that $\left.\lambda\right|_{M_{0}}$ and $\left.\lambda\right|_{M}$ are positive and large constant multiples of $\Lambda_{0}$ and $\Lambda_{\epsilon}$, respectively, where the constant can be chosen $\epsilon$-independent. Consider the Liouville completion $\widehat{W}$, obtained from $W$ by attaching one positive and one negative cylindrical end, and the natural extension of $\lambda$ to $\widehat{W}$. Choose a $\lambda$-compatible almost complex structure $J$, such that $J$ coincides with $J_{0}$ and $J_{1}$ on their respective ends and $J$ is generic along the main level. Consider also the compactification $\bar{W}^{\infty}$, obtained by adding $+\infty$ and $-\infty$ to $\widehat{W}$, on which we have extensions of both the symplectic form $d \lambda$ and $J$ (see [16] for more details on this construction).

As I have already described, there exists a distinguished ( $\mathbb{R}$-translation class of a) regular index $1 J_{0}$-holomorphic cylinder $u_{0}$, lying in the upper levels of $\widehat{W}$. Let $T$ be the total action of its two positive ends. Recall that by the model $\mathrm{B}$ construction, there is an action threshold $T_{\epsilon}>0$ such that $\lim _{\epsilon \rightarrow 0} T_{\epsilon}=+\infty$ and every $\Lambda_{\epsilon}$-closed orbit with action less than $T_{\epsilon}$ corresponds to a critical point in $M$ or lies along the spine $Y \times \mathcal{U}$. Observe that this construction also holds if we multiply the contact form by a constant positive number which is $\epsilon$-independent. Take $\epsilon>0$ small enough so that $T_{\epsilon}>T$. By Lemma 3.4, 
having attached a suitable exact symplectic cobordism to the concave end of $W$ prior to taking the completion, we can assume that $\left.\lambda\right|_{M}$ is a constant ( $\epsilon$-independent) multiple of the model B contact form $\Lambda_{\epsilon}$ corresponding to this particular $\epsilon>0$. By Stokes' theorem, the action of the Reeb orbits appearing in any building configuration arising as a limit of a curve in the moduli space of $u_{0}$ is bounded by $T_{\epsilon}$. Therefore, in what follows, I can make use of the discussion in Section 4.3, from which I gather that whenever this cylinder breaks in lower levels, the Reeb orbits obtained all correspond to critical points, and none lies over the spine.

Denote by $\overline{\mathcal{M}}$ the connected component containing $u_{0}$ of the nodal compactification of the space of finite-energy $J$-holomorphic curves in $\bar{W}^{\infty}$. It is a 1-dimensional compact manifold with boundary, having one boundary component corresponding to the projection of $u_{0}$ to $M_{0}$.

Observe first that no element of $\overline{\mathcal{M}}$ can break in an upper level. Indeed, by the aforementioned uniqueness theorem [16, Theorem 3.9] (more precisely, its adaptation as in the proof of [16, Theorem 5.2]), all of the upper components of a stable building $u \in \partial \overline{\mathcal{M}}$ lying in $\mathbb{R} \times M_{0}$ have to correspond to flow-line cylinders of a Morse function (which have nonnegative index). But then $u$ can only consist of an index 1 cylinder in the bottom end and a chain of index 0 cylinders on top of it, which are necessarily trivial. Since $u$ is stable, this configuration is not allowed unless $u$ coincides with its bottom level, but then $u$ is nonbroken.

Similarly, if an element in $\overline{\mathcal{M}}$ breaks with a nontrivial main-level component and no lower level ones, then uniqueness gives that there is only one possible breaking configuration. It consists of a single upper level, having an index 1 somewhere injective flow-line cylinder, together with a trivial (index zero) cylinder, and a nontrivial somewhere injective index 0 main-level component consisting of a cylinder with two positive ends, glued along simply covered Reeb orbits corresponding to critical points of Morse index 1 and 3. This configuration has a cancelling evil twin, since the index 1 component does (this is analogous to what we have already observed for model B), and it also consists of somewhere injective components. Therefore, we can glue this configuration to a unique cylinder. Then we obtain two 1-dimensional moduli spaces, each having an open end on each twin configuration. We can then canonically identify them along these open ends and obtain a new moduli space, which I still call $\overline{\mathcal{M}}$, for which we repeat this process of gluing further open ends corresponding to twin configurations.

Assume that an element in $\overline{\mathcal{M}}$ breaks in a lower level of $\bar{W}^{\infty}$, corresponding to the negative symplectisation of $M$. Since the cobordism is exact, there are no holomorphic caps, so that all components of all possible breaking configurations are still cylinders. Then I can argue similarly as before, but using an obstruction bundle. From the discussion in Section 4.3, every configuration has an obstruction bundle and comes with a twin configuration. Recall also that we are in the situation of Remark 3.17. We can choose finite and sufficiently large gluing parameters such that any configuration glues to holomorphic cylinders whose number is the algebraic count of zeroes of the section of the corresponding obstruction bundle. This number is independent of the gluing parameters, and is the opposite of what we would get if we were to glue starting from the twin configuration. Since we know that the original configuration glues at least once, its twin will also. 
The rest of the argument goes as before. Observe that we have not needed an abstract perturbation.

After these identifications, I will have constructed a compact 1-dimensional moduli space which has only one boundary component, which is a contradiction. This finishes the proof.

Acknowledgment I thank my $\mathrm{PhD}$ supervisor, Chris Wendl, for introducing me to this project and for his support and patience throughout its duration. To Richard Siefring, for very helpful conversations and for coauthoring [23, Appendix C]. To Janko Latschev and Kai Cieliebak, for going through the long process of reading [23]. To Patrick Massot, Sam Lisi, Michael Hutchings, Momchil Konstantinov and Zhengyi Zhou, for helpful conversations/correspondence on different topics. To the anonymous referee, for careful reading of previous versions and very detailed suggestions.

This research, forming part of my $\mathrm{PhD}$ thesis, was partly carried out in (and funded by) University College London in the UK and the Berlin Mathematical School in Germany.

\section{Appendix A. Superrigidity for punctured holomorphic curves}

In this appendix, I will derive Theorem 1.10, a general result bearing some independent interest. While I do not use it in this article, it will come as a by-product of Proposition A.1 and Theorem A.3.

For a holomorphic degree $d$ branched cover $\varphi:(\dot{S}, j) \rightarrow(\dot{\Sigma}, i)$ between punctured Riemann surfaces $\dot{S}=S \backslash \Gamma(S)$ and $\dot{\Sigma}=\Sigma \backslash \Gamma(\Sigma)$, define by

$$
\kappa(\varphi)=\sum_{w \in \Gamma(S)} \kappa_{w} \geq \# \Gamma(S)
$$

the total multiplicity of $\varphi$, where $\kappa_{w}=Z\left(d_{w} \varphi\right)+1$ is the multiplicity of $\varphi$ at the puncture $w$ (where $Z\left(d_{w} \varphi\right)$ is the vanishing order of $d \varphi$ at $w$ of the unique extension of $\varphi$ as a map $\varphi: S \rightarrow \Sigma$, preserving punctures). Observe that

$$
\kappa(\varphi)=\sum_{z \in \Gamma(\Sigma)}\left(\sum_{w \in \varphi^{-1}(z)} \kappa_{w}\right)=d \# \Gamma(\Sigma) .
$$

Therefore, $d \# \Gamma(\Sigma) \geq \# \Gamma(S)$, with equality if and only if there is no branching at the punctures. I will refer to the sum of the branching of $\varphi$ at interior points of $\dot{S}$ with the branching at the punctures as the total branching of $\varphi$.

Proposition A.1. Let $v:(\dot{\Sigma}, i) \rightarrow(W, J)$ be a somewhere injective possibly punctured holomorphic curve in a 4-dimensional symplectic cobordism, with index zero and with $\chi(v)=0$ (where $\chi(v):=\chi(\dot{\Sigma}))$. Assume that there exists a trivialisation $\tau$ for which the asympotics of $v$ have vanishing Conley-Zehnder index. Assume also that $v$ satisfies $\operatorname{ker} \mathbf{D}_{v}^{N}=0$. Then $\mathbf{D}_{u}^{N}$ is also injective for any multiple cover $u=v \circ \varphi$ of $v$, such that $\varphi$ has strictly positive total branching.

Remark A.2. Proposition A.1 does not apply (a priori) only to totally unbranched curves, and the unpunctured case recovers Wendl's results for the torus. It follows from the proof that we can also assume $\chi(v) \geq 0$. However, there are no holomorphic planes 
as in the hypothesis, since then $0=$ ind $\mathbf{D}_{v}^{N}=\chi(v)+2 c_{1}^{\tau}\left(N_{v}\right)=1+2 c_{1}^{\tau}\left(N_{v}\right)$, but $c_{1}^{\tau}\left(N_{v}\right)$ is an integer. We would then only obtain a result for spheres, which is already shown in [11] by a much shorter proof, where the injectivity of $\mathbf{D}_{v}^{N}$ is automatic and the conclusion holds for every multiple cover. Observe also that I have not assumed that $J$ is generic. For generic $J$, we can use Theorem A.3 to obtain the conclusion for every multiple cover. By a small modification of the proof, we can drop the index zero condition as well as the condition on $\chi(v)$, and impose instead that $c_{1}^{\tau}\left(N_{v}\right)=0$ for the given trivialisation $\tau$. However, then ind $\mathbf{D}_{v}^{N}=\chi(v)$, and if this is negative, then generically $v$ does not exist. We then only obtain a potentially interesting result for index 1 planes or index 2 spheres, with $c_{1}^{\tau}\left(N_{v}\right)=\mu_{C Z}^{\tau}=0$ for some $\tau$.

The proof follows by an adaptation of Hutchings' magic trick [8], as used in [11, Proposition 7.2] in the case of closed curves. I redo Wendl's computation, and the unpunctured case recovers Wendl's results for the torus.

In the proof of Proposition A.1, I will use Siefring's intersection pairing and the adjunction formula for punctured curves. I refer to [21] (especially Theorem 4.4) for the statement and details on the necessary definitions. In particular, the symbol $\bar{\sigma}(u)$ denotes the spectral covering number of $u$. This is defined as the sum over all punctures of the covering multiplicity of any extremal eigenfunction of the associated asymptotic operators. Here, the positive (resp. negative) extremal eigenfunctions are defined as those whose eigenvalue is the smallest (resp. largest) positive (resp. negative) eigenvalue. The covering multiplicity is independent of the choice of eigenfunction (see [21, p. 59]). The winding number of any extremal eigenfunction is referred to as an extremal winding number.

Since the multiplicity of the eigenfunction divides that of the asymptotic orbit, we have $\bar{\sigma}(u)=\# \Gamma(u)$ whenever the asymptotics of $u$ are simply covered. Also, the symbol $\delta(u)$ denotes the algebraic count of double points and critical points of $u$. It vanishes if and only if $u$ is embedded. The symbol $\delta_{\infty}(u)$ is the algebraic count of 'hidden double points at infinity'. If different punctures of $u$ asymptote different orbits, and all of them are simply covered, then this number vanishes (see [21, Theorem 4.17] for the general case).

Proof of Proposition A.1. As explained in, for example, [11, Appendix], an almost complex structure $J_{N}$ can be constructed on the total space of the normal bundle $\pi$ : $N_{v} \rightarrow \dot{\Sigma}$, such that there is a one-to-one correspondence between holomorphic curves $u_{\eta}:(\dot{S}, j) \rightarrow\left(N_{v}, J_{N}\right)$ and sections $\eta \in \operatorname{ker} \mathbf{D}_{u}^{N}$ along the holomorphic branched cover $\varphi=$ $\pi \circ u_{\eta}:(\dot{S}, j) \rightarrow(\dot{\Sigma}, i)$, where $u=v \circ \varphi$ is a branched cover of $v$ (and $\eta$ is identified with $u_{\eta}$ as maps).

Fix $u=v \circ \varphi$. I will assume nothing on $\chi(v)$ until the very end. Let $d:=\operatorname{deg}(\varphi) \geq 1$. I proceed by induction on $d$. The base case $d=1$ holds by assumption, so take $d>1$; I assume that every branched cover $u^{\prime}$ of $v$ with degree strictly less than $d$ has $\operatorname{ker} \mathbf{D}_{u^{\prime}}^{N}=0$. Assume by contradiction that there exists a nonzero $\eta \in \operatorname{ker} \mathbf{D}_{u}^{N}$, and take the corresponding $u_{\eta}$. We can view $v$ as a $J_{N}$-holomorphic embedding inside $N_{v}$ (as the zero section), and we have that $u_{\eta}$ is homologous to $d[v]$, where $[v]$ is a relative homology class. Observe that $v$, as a holomorphic map to $N_{v}$, is embedded, and each 
of its asymptotics is distinct and simply covered, so that we get $\delta(v)=\delta_{\infty}(v)=0$ and $\bar{\sigma}(v)=\# \Gamma(v)$. Moreover, we can write $u_{\eta}=\widehat{u} \circ \psi$, where $\widehat{u}$ is somewhere injective and $\psi:(\dot{S}, j) \rightarrow\left(\dot{S}^{\prime}, j^{\prime}\right)$ is a branched cover. But then $\widehat{u}$ is of the form $\widehat{u}=u_{\eta^{\prime}}^{\prime}$, where $u^{\prime}=v \circ \varphi^{\prime}$, with $\varphi^{\prime}=\pi \circ \widehat{u}$ and $\eta^{\prime} \in \operatorname{ker} \mathbf{D}_{u^{\prime}}$, satisfying $\varphi^{\prime} \circ \psi=\varphi, \eta=\eta^{\prime} \circ \psi$. Then $d=$ $\operatorname{deg}\left(\varphi^{\prime}\right) \operatorname{deg}(\psi)$. If $\operatorname{deg}(\psi)>1$, then $\operatorname{deg}\left(\varphi^{\prime}\right)<d$, and by the induction hypothesis applied to $u^{\prime}$, we have that $\eta^{\prime}=0$ and so $\eta=0$. Therefore, we can assume that $u_{\eta}$ is somewhere injective.

Since the asymptotics of $v$ have vanishing Conley-Zehnder index, their extremal winding numbers also vanish. Therefore the extremal eigenfunctions are constant, so that their covering multiplicity coincides with that of the corresponding asymptotic orbit. If we denote by $\kappa\left(u_{\eta}\right)$ the sum of all the multiplicities of the asymptotics of $u_{\eta}$ (which coincides with $\kappa(u):=\kappa(\varphi)$ ), we obtain that the spectral covering number $\bar{\sigma}\left(u_{\eta}\right)=\kappa\left(u_{\eta}\right)=\kappa(u)=d \# \Gamma(v)$.

We can then compute its Siefring self-intersection number:

$$
\begin{aligned}
u_{\eta} * u_{\eta} & =2\left(\delta\left(u_{\eta}\right)+\delta_{\infty}\left(u_{\eta}\right)\right)+c_{1}^{\tau}\left(u_{\eta}^{*} T N_{v}\right)-\chi\left(u_{\eta}\right)+\bar{\sigma}\left(u_{\eta}\right)-\# \Gamma\left(u_{\eta}\right) \\
& =2\left(\delta\left(u_{\eta}\right)+\delta_{\infty}\left(u_{\eta}\right)\right)+d c_{1}^{\tau}\left(v^{*} T N_{v}\right)-\chi\left(u_{\eta}\right)+d \# \Gamma(v)-\# \Gamma(u) .
\end{aligned}
$$

Moreover, Riemann and Hurwitz give

$$
\chi\left(u_{\eta}\right)=\chi(u)=d \chi(v)-Z(d \varphi) .
$$

Identifying the normal bundle to $v$ inside $N_{v}$ with $N_{v}$ itself, the adjunction formula then produces

$$
c_{1}^{\tau}\left(v^{*} T N_{v}\right)=\chi(v)+c_{1}^{\tau}\left(N_{v}\right)
$$

Moreover, we have that

$$
0=\operatorname{ind} \mathbf{D}_{v}^{N}=\chi(v)+2 c_{1}^{\tau}\left(N_{v}\right)
$$

Using (32) and the vanishing of the Conley-Zehnder indices, we obtain

$$
\begin{aligned}
u_{\eta} * u_{\eta} & =d^{2} v * v \\
& =d^{2}\left(c_{1}^{\tau}\left(v^{*} T N_{v}\right)-\chi(v)\right) \\
& =d^{2} c_{1}^{\tau}\left(N_{v}\right) \\
& =-\frac{d^{2}}{2} \chi(v) .
\end{aligned}
$$

Combining (29), (30), (31), (32) and (33), we get

$$
\begin{aligned}
0 & \leq 2\left(\delta\left(u_{\eta}\right)+\delta_{\infty}\left(u_{\delta}\right)\right) \\
& =-\frac{d^{2}}{2} \chi(v)-d\left(\chi(v)+c_{1}^{\tau}\left(N_{v}\right)\right)+d \chi(v)-Z(d \varphi)+\# \Gamma(u)-d \# \Gamma(v) \\
& =\frac{d(1-d)}{2} \chi(v)-Z(d \varphi)+\# \Gamma(u)-d \# \Gamma(v) .
\end{aligned}
$$

If we assume that $\chi(v)=0$ (or $\chi(v) \geq 0$ ), this is negative, and we obtain a contradiction. 
In order to obtain Theorem 1.10, we need another result. Recall that a closed hyperbolic Reeb orbit is one whose linearised return map does not have eigenvalues in the unit circle. In dimension 3, if a closed Reeb orbit has even Conley-Zehnder index (in one and hence every trivialisation), then it is hyperbolic.

Theorem A.3. For generic $J$, all index 0 punctured holomorphic curves in a 4dimensional symplectic cobordism, which are unbranched multiple covers, have zero Euler characteristic and have all their asymptotics hyperbolic, are Fredholm regular.

(This theorem comes from a private communication from C. Wendl: January 2018.) This is proved by the same arguments as in [11] (which only deals with closed curves), and I will not include a proof. Note that Wendl's article, which was withdrawn at some point due to a gap in a lemma, has recently been fixed [18]. The main technicality has to do with the failure of Petri's condition in general (see [11] for a definition). Nevertheless, it does hold for generic $J$. In the present situation, the assumptions in Theorem A.3 bypass the need to consider these technicalities. Indeed, for a curve $u$ as in Theorem A.3, the normal Cauchy-Riemann operator $\mathbf{D}_{u}^{N}$ is defined on a line bundle $N_{u}$ whose adjusted first Chern number is nonpositive: we have $2-2 g-\# \Gamma=0$, so that $2-2 g-\# \Gamma_{\text {odd }}=\# \Gamma_{\text {even }}$, and therefore

$$
2 c_{1}\left(N_{u}, u\right)=\operatorname{ind}(u)-2+2 g+\# \Gamma_{\text {even }}=-\# \Gamma_{\text {odd }} \leq 0 .
$$

This means either that $\operatorname{ker} \mathbf{D}_{u}^{N}=0$ or that every element in $\operatorname{ker} \mathbf{D}_{u}^{N}$ is nowhere vanishing. Therefore Petri's condition is vacuously satisfied.

With this said, [11, Theorem D] needs to be generalised to the setting of asymptotically cylindrical curves in cobordisms. This is mostly a matter of putting it in the proper functional-analytic setup for punctured curves, and almost nothing else changes. Since all orbits are hyperbolic, Fredholm indices of multiple covers are related to indices of the underlying somewhere injective curves via the same multiplicative relations (involving the Riemann-Hurwitz formula) as in the closed case. In fact, the multiplicativity of the Conley-Zehnder indices is all that is needed. With that in mind, the same dimensioncounting argument as in [11, Theorem B] works in the case of hyperbolic punctures.

Proof of superrigidity and Theorem 1.10. In the case where $v$ is as in the hypotheses of Theorem 1.10, for generic $J$ we can assume it is immersed and regular. Since ind $\mathbf{D}_{v}^{N}=0$, then $\operatorname{ker} \mathbf{D}_{v}^{N}=0$ by regularity, and so the hypotheses of Proposition A.1 are satisfied for generic $J$. If we also assume that its totally unbranched covers satisfy the conclusion of the proposition (which is also a generic condition by Theorem A.3), the result follows.

\section{Appendix B. Lutz-Mori twists and Giroux torsion}

This appendix is devoted to describing Lutz-Mori twists as defined in [16] and using them in model A of [18], as described in the introduction. In the present case, we can use cylindrical contact homology to distinguish the twisted model A contact structures for different amounts of twisting - something which has been done already in [16, Theorem 8.13] for very similar models, so I will not include any details (see [16, App. A]). 


\section{B.1. The twisted contact structures}

Recall that a hypersurface $H \subseteq M$ in a contact manifold $(M, \xi)$ is a $\xi$-round hypersurface modeled on $\left(Y, \xi_{0}\right)$ if it is transverse to $\xi$ and admits an orientation preserving identification with $S^{1} \times Y$, for some contact manifold ( $\left.Y, \xi_{0}\right)$, such that $\xi \cap T H=T S^{1} \oplus \xi_{0}$. This notion is defined in [16].

Take $\left(\alpha_{-}, \alpha_{+}\right)$, a Liouville pair on $Y^{2 n-1}$, and denote $\xi_{ \pm}=\operatorname{ker} \alpha_{ \pm}$. Consider the Giroux $2 \pi l$-torsion domain modeled on $\left(Y, \alpha_{-}, \alpha_{+}\right)$, which is the contact manifold

$$
G T_{l}^{+}:=\left(Y \times[0,2 \pi l] \times S^{1}, \xi_{G T}=\operatorname{ker} \lambda_{G T}\right),
$$

where

$$
\lambda_{G T}=\frac{1+\cos (r)}{2} \alpha_{+}+\frac{1-\cos (r)}{2} \alpha_{-}+\sin (r) d \theta,
$$

both of whose boundary components are $\xi_{G T}$-round hypersurfaces modeled on $\left(Y, \xi_{+}\right)$. We can also consider the modified version given by

$$
G T_{l}^{-}:=\left(Y \times[\pi / 2,2 \pi l+\pi / 2] \times S^{1}, \xi_{G T}=\operatorname{ker} \lambda_{G T}\right),
$$

whose boundaries are now modeled on $\left(Y, \xi_{-}\right)$.

For the model A version of the contact structure $\xi_{k}$ in $M=Y \times \Sigma$ (in the same isotopy class as my model B contact structures), we have that $\xi_{k}=\xi_{ \pm} \oplus T \Sigma_{ \pm}$over the regions $Y \times \Sigma_{ \pm}$. Therefore, we obtain ( $k$ copies of) round hypersurfaces $H_{ \pm}$modeled on $\left(Y, \xi_{ \pm}\right)$, corresponding respectively to the boundary components of $Y \times \Sigma_{ \pm}$. We can therefore perform an $l$-fold Lutz-Mori twist along the $H_{ \pm}$. This can be done in two equivalent ways. The first consists of cutting my model along each of the $k$ copies of $H_{-}$and gluing a copy of $G T_{l}^{-}$; the second consists doing the same for $H_{+}$, along which we glue $G T_{l}^{+}$. This yields a manifold which is diffeomorphic to $M$, with a contact structure on $M$ which I denote $\xi_{k}^{l}$. The two ways are equivalent, since the contact structure $\xi_{k}$ along the cylindrical region $M_{Y}$ (lying in between $H_{-}$and $H_{+}$) is itself isotopic to a 'half' Giroux torsion domain of the form $\left(Y \times[\pi / 2,2 \pi], \xi_{G T}\right)$, as is easily checked. The contact structure $\xi_{k}^{l}$ is homotopic as almost contact structures to the original contact structure $\xi_{k}$. Since $\xi_{k}^{l}$ has Giroux torsion, Theorem 1.4 implies that it has algebraic 1-torsion (for $l>0$ ), so that they are indistinguishable from this invariant alone. Corollary 1.9 follows from Theorem 1.5. On the other hand, one can use cylindrical contact homology to distinguish the $\xi_{k}^{l}[16]$.

\section{References}

[1] F. Bourgeois, A Morse-Bott approach to contact homology, Fields Inst. Commun. 35 (2003), 55-77.

[2] M. Chas And D. Sullivan, String topology, Preprint, 1999, arXiv:math/9911159.

[3] K. Cieliebak, Nonlinear functional analysis, lecture notes, https://www.math.uniaugsburg.de/prof/geo/Books-and-Scripts/nonlin_10_.pdf.

[4] K. CieliebaK and J. Latschev, The role of string topology in symplectic field theory, in New Perspectives and Challenges in Symplectic Field Theory, CRM Proc. Lect. Notes 49 (Providence, American Mathematical Society, 2009), 113-146.

[5] Y. Eliashberg, A. Givental and H. Hofer, Introduction to symplectic field theory, Geom. Funct. Anal. special volume (2000), 560-673. 
[6] H. Hofer, K. Wysocki And E. Zehnder, Integration theory on the zero sets of polyfold Fredholm sections, Math. Ann. 346(1) (2010), 139-198.

[7] M. Hutchings, A magic trick for defining obstruction bundles, http://floerhomology. wordpress.com/2013/09/04/.

[8] M. Hutchings, Gluing a flow line to itself, https://floerhomology.wordpress.com/2014/ 07/14/gluing-a-flow-line-to-itself/.

[9] M. Hutchings and C. Gerig, Obstruction bundle gluing, notes for IHES Moduli Conference July 2015, https://www.math.ias.edu/ joelfish/Hutchings_full.pdf.

[10] M. Hutchings and C. Taubes, Gluing pseudoholomorphic curves along branched covered cylinders: I, J. Symplectic Geom. 5(1) (2007), 43-137.

[11] M. Hutchings And C. Taubes, Gluing pseudoholomorphic curves along branched covered cylinders: II, J. Symplectic Geom. 7(1) (2009), 29-133.

[12] C. Kutluhan, G. Matic, J. Van Horn-Morris and A. Wand, Algebraic torsion via Heegaard Floer homology, Preprint, 2015, arXiv:1503.01685v3.

[13] J. Latschev and C. Wende, Algebraic torsion in contact manifolds, Geom. Funct. Anal. 21(5) (2011), 1144-1195.

[14] S. Lisi, J. VAn Horn-Morris and C. Wend, On symplectic fillings of spinal open book decompositions I: Geometric constructions, Preprint, 2018, arXiv:1810.12017.

[15] R. Lutz, Structures de contact sur les fibrès principaux en cercles de dimension trois, Ann. Inst. Fourier (Grenoble) 27(3) (1977), ix, 1-15 (French, with English summary).

[16] P. Massot, K. NiederkrüGer and C. Wend, Weak and strong fillability of higher dimensional contact manifolds, Inv. Math. 192(2) (2013), 287-373.

[17] D. McDuff, Symplectic manifolds with contact type boundaries, Inv. Math. 103(1) (1991), 651-671.

[18] A. Moreno, Algebraic and Giroux torsion in higher-dimensional contact manifolds, Preprint.

[19] A. Moreno, Algebraic Torsion in Higher-Dimensional Contact Manifolds, PhD thesis, Humboldt Universität zu Berlin, 2018, arXiv:1711.01562.

[20] A. Moreno And R. Siefring, Holomorphic curves in the presence of holomorphic hypersurface foliations, Preprint, 2019, arXiv:1902.02700.

[21] A. Mori, Reeb foliations on $S^{5}$ and contact 5-manifolds violating the Thurston-Bennequin inequality, Preprint, 2009, arXiv:0906.3237.

[22] K. Niederkrüger And C. Wende, Weak symplectic fillings and holomorphic curves, Ann. Sci. Éc. Norm. Supér. (4) 44(5) (2011), 801-853.

[23] E. Schoenfeld, Higher Symplectic Field Theory Invariants for Cotangent Bundles of Surfaces, PhD thesis, Stanford University.

[24] R. Siefring, Intersection theory of punctured pseudoholomorphic curves, Geom. Topol. 15(4) (2011), 2351-2457.

[25] R. Siefring, Finite-energy pseudoholomorphic planes with multiple asymptotic limits, Math. Ann. 368(1-2) (2017), 367-390.

[26] R. Siefring, Symplectic field theory and stable hamiltonian submanifolds: Intersection theory, In preparation.

[27] W. Thurston, Some simple examples of symplectic manifolds, Proc. Amer. Math. Soc. 55 (1976), 467-468.

[28] C. Wend, Automatic transversality and orbifolds of punctured holomorphic curves in dimension four, Comment. Math. Helv. 85(2) (2010), 347-407.

[29] C. WENDL, Lectures on symplectic field theory, Lecture notes, arXiv:1612.01009v2.

[30] C. WendL, A hierarchy of local symplectic filling obstructions for contact 3-manifolds, Duke Math. J. 162(12) (2013), 2197-2283. 
[31] C. WendL, Strongly fillable contact manifolds and $J$-holomorphic foliations, Duke Math. J. 151(3) (2010), 337-384.

[32] C. WendL, Transversality and super-rigidity for multiply covered holomorphic curves, Preprint, 2016, arXiv:1609.09867.

[33] C. Wende, Contact 3-manifolds, holomorphic curves and intersection theory, Lecture notes, arXiv:1706.05540.

[34] C. WendL, Super-rigidity is fixed, https://symplecticfieldtheorist.wordpress.com/2019/ 05/27/super-rigidity-is-fixed/.

[35] C. Wend, Lectures on Holomorphic Curves in Symplectic and Contact Geometry, https://www.mathematik.hu-berlin.de/ wendl/pub/jhol_bookv33.pdf. 